\title{
Genetic inactivation of zinc transporter SLC39A5 improves liver function and hyperglycemia in obesogenic settings
}

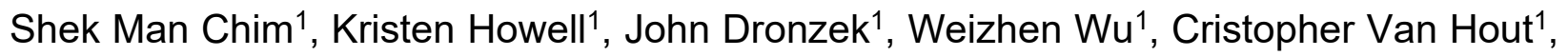
Manuel Allen Revez Ferreira ${ }^{1}$, Bin $\mathrm{Ye}^{1}$, Alexander $\mathrm{Li}^{1}$, Susannah Brydges ${ }^{2}$, Vinayagam Arunachalam ${ }^{1}$, Anthony Marcketta ${ }^{1}$, Adam E Locke ${ }^{1}$, Jonas Bovijn ${ }^{1}$, Niek Verweij ${ }^{1}$, Tanima De ${ }^{1}$, Luca Lotta ${ }^{1}$, Lyndon Mitnaul ${ }^{1}$, Michelle G. LeBlanc ${ }^{1}$, Regeneron Genetics Center ${ }^{1}$, DiscovEHR collaboration ${ }^{3}$, David Carey $^{3}$, Olle Melander ${ }^{4}$, Alan Shuldiner ${ }^{1}$, Katia Karalis $^{1}$, Aris N. Economides ${ }^{1,2 \dagger}$ and Harikiran Nistala ${ }^{1 \dagger}$

1. Regeneron Genetics Center, 777 Old Saw Mill River Road, Tarrytown NY

2. Regeneron Pharmaceuticals, Inc., 777 Old Saw Mill River Road, Tarrytown NY

3. Geisinger Health System, Danville, PA

4. Department of Clinical Sciences in Malmö, Lund University, Malmö, Sweden

† Corresponding Authors:

Harikiran Nistala, Ph.D

Regeneron Genetics Center, Regeneron Pharmaceuticals, Inc.

777 Old Saw Mill River Road, Tarrytown NY 10591

Phone: (732) 543 6367; Email: kiran.nistala@gmail.com

Aris N. Economides, Ph.D.

Regeneron Genetics Center, Regeneron Pharmaceuticals, Inc.

777 Old Saw Mill River Road, Tarrytown NY 10591

Email: aris.economides@regeneron.com

\section{Conflict of Interest Statement:}

S.M.C., K.H., W.W., M.A.R.F, B.Y., A.L., S.B., A.M., A.E.L., J.B., N.V., T.D., L.L., L.M., M.G.L., A.S., K.K., A.N.E., are full-time employees of the Regeneron Genetics Center or Regeneron Pharmaceuticals Inc. and hold stock options/restricted stock as part of compensation. 
Financial support: This work was supported by Regeneron Pharmaceuticals.

Author contributions: H.N., A.N.E. and S.M.C. designed the study. S.M.C., K.H., J.D., W.W. and H.N. generated and analyzed the data. H.N., C.V.H., M.A.R.F., B.Y., A.L., S.B., V.A., A.M., A.E.L., J.B., N.V., T.D., L.L., L.M., M.G.L., D.C., O.M., A.S., DiscovEHR and Regeneron Genetics Center performed the human genetics data collection and analysis. S.M.C., K.K., A.N.E. and H.N. wrote the manuscript. 


\section{Graphical abstract:}

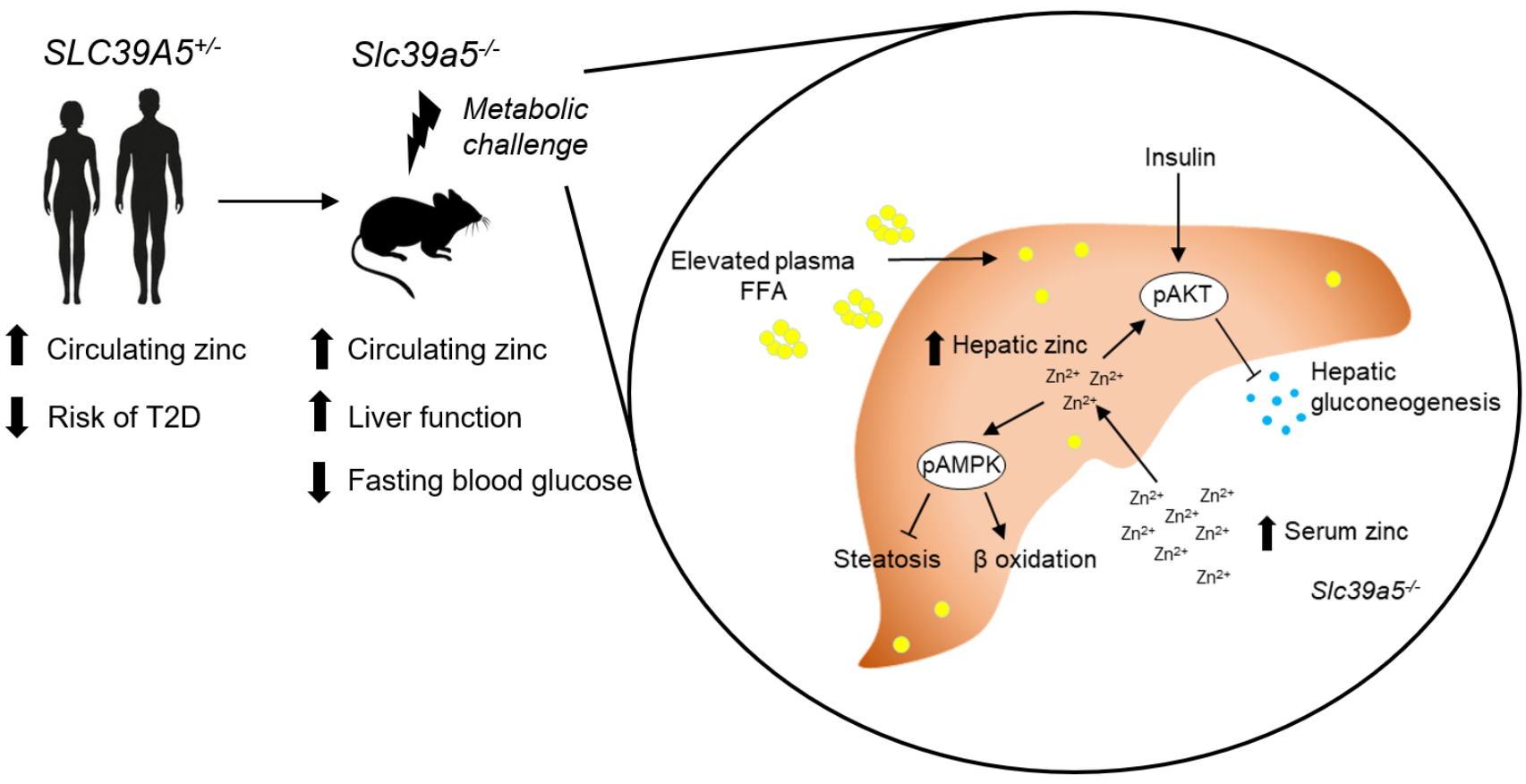

\section{Manuscript Highlights:}

- Heterozygous loss-of-function mutations in SLC39A5 associated with elevated circulating zinc levels and nominal reduction in type II diabetes risk in humans.

- Loss of SIc39a5 results in elevated circulating and hepatic zinc levels in mice.

- Mice lacking Slc39a5 function are protected against hepatic steatosis and hyperglycemia resulting from diet-induced obesity or leptin-receptor deficiency and display reduced hepatic inflammation and fibrosis resulting from diet-induced $\mathrm{NASH}$.

- Loss of SIc39a5 function results in hepatic AMPK and AKT activation.

- SLC39A5 is a potential therapeutic target for fatty liver disease and type II diabetes. 


\section{Abstract \\ Background \& Aims:}

Recent studies have revealed a role for zinc in insulin secretion and systemic glucose homeostasis. Randomized placebo-controlled zinc supplementation trials have demonstrated improved glycemic traits in patients with type II diabetes (T2D). Moreover, carriers of rare loss-of-function variants in the zinc efflux transporter SLC30A8 have been reported to reduce T2D risk. Despite this accumulated evidence, mechanistic understanding of how zinc influences systemic glucose homeostasis and consequently T2D risk remains unclear.

\section{Methods:}

To further explore the relationship between zinc and metabolic traits, we searched the exome database of the Regeneron Genetics Center-Geisinger Health System DiscovEHR cohort for genes that regulate zinc levels and associate with changes in metabolic traits. We then explored our main finding using in vitro and in vivo models.

\section{Results:}

We identified rare loss-of-function (LOF) variants (MAF<1\%) in Solute Carrier Family 39 , Member 5 (SLC39A5) associated with increased circulating zinc $\left(p=4.9 \times 10^{-4}\right)$. Transancestry meta-analysis across four studies exhibited nominal association of SLC39A5 LOF variants with decreased T2D risk (OR 0.82, 95\% Cl 0.68-0.99, $p=3.7 \times 10^{-2}$ ). To explore the mechanistic aspects of these associations, we generated mice lacking Slc39a5. S/c39a5 $/-$ mice display improved liver function and reduced hyperglycemia when challenged with congenital or diet-induced obesity. These improvements result from elevated hepatic zinc levels and concomitant activation of hepatic AMPK and AKT signaling, in part due to zinc mediated inhibition of hepatic protein phosphatase activity. Furthermore, under conditions of diet-induced non-alcoholic steatohepatitis (NASH), S/c39a5 $\%$ mice display significantly attenuated fibrosis and inflammation.

\section{Conclusions:}

Taken together, these results suggest SLC39A5 as a potential therapeutic target for nonalcoholic fatty liver disease (NAFLD) and consequent metabolic derangements including T2D. 
medRxiv preprint doi: https://doi.org/10.1101/2021.12.08.21267440; this version posted December 9, 2021. The copyright holder for this preprint (which was not certified by peer review) is the author/funder, who has granted medRxiv a license to display the preprint in perpetuity.

\section{Lay summary:}

Zinc is an essential trace element in human health. We identified mutations in a zinc transporter gene, SLC39A5 that are associated with increased circulating zinc and nominal reduction in type II diabetes risk in humans. To understand this connection we generated mice lacking this gene and showed that they have improved liver function and reduced hyperglycemia under obesogenic conditions and also when given a diet that causes fatty liver disease. Our observations suggest that pharmacological inhibition of SLC39A5 may be a potential therapeutic avenue for type II diabetes and non-alcoholic fatty liver disease. 


\section{Introduction}

Zinc $\left(\mathrm{Zn}^{2+}\right)$ is an essential trace element with established roles in enzyme biochemistry and other biological processes. Hence, robust homeostatic mechanisms have evolved to maintain physiological levels of zinc and coordinate spatiotemporal demands across various tissues ${ }^{1}$. Metal transporter proteins encoded by solute carrier (SLC) gene families SLC30 (zinc transporter, ZnT) and SLC39 (Zrt- and Irt-like protein, ZIP) facilitate zinc homeostasis by mediating cellular $\mathrm{Zn}^{2+}$ efflux and uptake respectively ${ }^{2}$.

Converging lines of evidence have shown that zinc plays a crucial role in insulin secretion and glucose metabolism. For example, increasing zinc intake improves glycemic control in prediabetics and patients with $\mathrm{T}^{2} \mathrm{D}^{3}$. Furthermore, loss-of-function (LOF) variation in SLC30A8 (encoding a pancreatic islet zinc transporter ZnT8) in humans associates with reduced glucose levels and a 65\% reduction in T2D risk resulting from enhanced insulin responsiveness to glucose combined with increased pro-insulin processing $^{4,5}$. To further explore mechanisms underlying the T2D-protective role of zinc and identify additional genetic determinants influencing systemic zinc homeostasis, we tested loss-of-function variation in zinc transporters for association with circulating zinc and T2D risk and identified rare putative LOF (pLOF) variants (MAF<1\%) in SLC39A5 associated with elevated circulating zinc $\left(p=4.9 \times 10^{-4}\right)$. We demonstrate that the identified pLOF variants encode non-functional SLC39A5 proteins. Using Slc39a5 demonstrate that loss of S/c39a5 results in lower glucose levels due to elevated hepatic zinc, and has protective effects in models of congenital, diet-induced obesity, and NASH. These effects appear to be mediated by elevated hepatic zinc levels and concomitant activation of hepatic AMPK and AKT signaling, thereby uncovering a mechanistic basis for zinc induced glucose control and indicating that SLC39A5 inhibition may hold therapeutic potential in NAFLD and T2D.

\section{Materials and methods}

Slc39a5/- and Lepr/- mouse lines were created using VelociGene ${ }^{\circledR}$ technology $^{6}$.

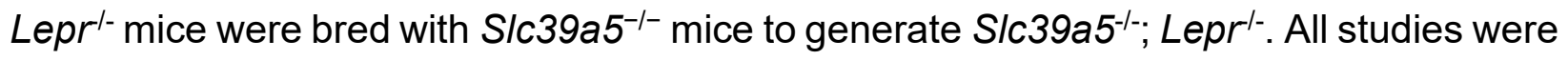
performed in both sexes. For HFFD study, ten-week-old mice were fed HFFD diet (46kcal\% Fat, 30kcal\% Fructose, TestDiet 5WK9) or control diet (TestDiet 58Y2) for 30 
weeks. For NASH study, ten week old mice were fed NASH diet (40kcal\% Fat, 20 kcal\% Fructose and 2\% Cholesterol, ResearchDiets D09100310) or control diet (ResearchDiets D09100304) for 40 weeks. All mice used in this study were housed in pathogen-free environment at Regeneron Pharmaceuticals Inc. animal research facility. Sterile water and chow were provided ad libitum. All experimental protocols and tissue harvesting procedures were performed with Regeneron Pharmaceuticals Inc., Institutional Animal Care and Use Committee (IACUC) approval. Data are presented as box plots with individual values. Statistical analysis was performed using one-way or two-way ANOVA, followed by post hoc Tukey's tests. Statistical significance reported when $p<0.05$. Sample sizes, statistical test and significance are described in each figure legend.

Full Methods and any associated references are available in supplementary information.

\section{Results}

Rare loss-of-function variants in SLC39A5 associate with elevated serum zinc and type II diabetes

Using exome sequence data from participants of European ancestry in the Regeneron Genetics Center-Geisinger Health System DiscovEHR study, we identified rare pLOF variants (MAF<1\%) in SLC39A5 associated with increased circulating zinc levels in heterozygous carriers $\left(p=4.9 \times 10^{-4}\right.$; Fig. 1A). We also tested rare LOF variants in SLC39A5 for association with T2D in a multi-ethnic meta-analysis of four studies (UK Biobank, DiscovEHR, Mount Sinai's BioMe study, and Malmö Diet and Cancer Study), totaling $>62,000$ cases and $>518,000$ controls, and found them to be nominally associated with protection from T2D $\left(\mathrm{OR} 0.82,95 \% \mathrm{Cl} 0.68-0.99, p=3.7 \times 10^{-2}\right.$, Fig. 1B). Using serum call back analyses, we confirmed that circulating zinc levels in SLC39A5 heterozygous loss of function carriers are elevated by $12 \%$ as compared to age, sex, BMI-matched reference controls ( $p=0.0024 ;$ Fig. 1C). Furthermore, analyses of insulin production (proinsulin/insulin), insulin clearance (insulin/c-peptide ratio) and blood glucose in these samples demonstrated no differences based on genotype (Fig. 1D-I and Suppl. Table 1). These results, in conjunction with lack of SLC39A5 expression in pancreatic $\beta$-cells ${ }^{7,8}$, suggested that SLC39A5 does not influence pancreatic $\beta$-cell development or function. 
To test whether the pLOF variants result in loss of protein function, we first examined their expression and cellular localization by immunofluorescence and flow cytometry. In these analyses we included several observed pLOF variants: p.Y47*(c.141C>G), p.R311*(c.931C>T), and p.R322*(c.964C>T). Bicistronic (IRESDsRED) mammalian expression constructs encoding untagged wild-type or SLC39A5 muteins $\left(Y 47^{*}, \mathrm{R} 311^{*}, \mathrm{R} 322^{*}\right.$,) were transfected into HEK293 cells (Fig. S1A-E). Consistent with previous reports ${ }^{9}$, flow cytometry and immunofluorescence analyses at steady state demonstrated that wild-type SLC39A5 localized to the cell surface (Fig. S1A and B). In contrast, localization of variants $Y 47^{*}, \mathrm{R} 311^{*}, \mathrm{R} 322^{*}$ to the cell surface was reduced by $\sim 91 \%, 98 \%$ and $99 \%$ respectively (Fig. S1D). To assess the zinc transport function of these variants, we leveraged a zinc-dependent transactivation assay using a metal regulatory element (MRE) responsive luciferase reporter. Wild-type SLC39A5 resulted in dose-dependent activation of the reporter to $\mathrm{Zn}^{2+}$ (an effect that was attenuated by zinc chelator N,N,N',N'-Tetrakis(2-pyridylmethyl)ethylenediamine) (Fig. S1C), whereas variants $Y 47^{*}, \mathrm{R} 311^{*}, \mathrm{R} 322^{*}$ failed to mediate a response (Fig. S1D and E). Therefore, variants $\mathrm{Y} 47^{*}, \mathrm{R} 311^{*}, \mathrm{R} 322^{*}$ encode non-functional proteins. Their association with elevated serum zinc levels in the corresponding carriers is consistent with the proposed role of SLC39A5 in maintaining systemic zinc homeostasis by facilitating efflux of excess serosal zinc into the gut lumen ${ }^{9}$.

\section{Slc39a5 homozygous-null mice display elevated serum and tissue zinc}

To investigate the role of Slc39a5 in glucose homeostasis in vivo, we generated Slc39a5-null mice (Fig. S2A). The resulting S/c39a5-/ mice completely lacked S/c39a5 transcript and protein in their duodenum and liver (Fig. S2B and C), two tissues with documented expression of SLC39A5. Consistent with our observations in human heterozygous LOF carriers, S/c39a5+/- mice had elevated circulating zinc levels ( 26\% in female and $\sim 23 \%$ in male) compared to wildtype littermates. The elevation in circulating zinc was greatly accentuated in S/c39a5 $/-$ mice ( $280 \%$ in females and $\sim 227 \%$ in males) compared to wild-type littermates (Fig. 2A). S/c39a5 $5^{-/}$mice displayed normal fecundity and had no overt phenotypes even at 22 months of age. Elemental analyses of major organs (in both sexes) revealed that S/c39a5-/- mice had significantly elevated zinc levels 
in the liver, bone, kidneys and brain, and lower levels in the pancreas (Fig. 2B and Suppl. Table 2). These phenotypes are consistent with previously reported Slc39a5 knockout mouse models ${ }^{10,11}$. No differences in magnesium, iron, copper, cobalt or calcium were observed in liver (Fig. S10C and F). Serum chemistry analysis in adult mice (10 months, both sexes) demonstrated no differences in pancreatic amylase, renal function parameters (blood urea nitrogen, creatinine, total protein and uric acid), electrolytes (chloride, potassium and sodium), and liver enzymes (alanine aminotransferase; ALT and aspartate aminotransferase; AST) (Suppl. Table 3), suggesting that the observed changes in tissue zinc levels are physiologically inert at this age. Unlike S/c39a5\%- mice, S/c39a5 $5^{+-}$mice showed no changes in tissue zinc levels despite elevation in serum zinc indicating that the free exchangeable pool of serum zinc in the Slc39a5 $5^{+/}$mice is not sufficient to alter zinc balance within tissues (Suppl. Table 2). Conservation at the protein level ( 83.5\% identity), similar postnatal expression ${ }^{12}$ and preserved function between mouse and human orthologs suggest that S/c39a5-/ mice are a valid model to explore the observed subthreshold T2D protective effect of SLC39A5 LOF alleles in humans.

\section{Loss of S/c39a5 results in reduced fasting blood glucose in congenital and diet- induced obesity models}

To assess whether disruption of Slc39a5 function improves glycemic traits in mice, we challenged the Slc39a5\%- mice with well-established models of congenital (leptinreceptor deficiency; Lepr/- mice) or diet induced obesity ${ }^{13,14}$. Loss of Slc39a5 did not alter body weight in either model (Fig. 3A, D, G, J and Fig. S5A, S6A, S7A, S8A). S/c39a5-/;Lepr/- mice or Slc39a5/- mice on high fat high fructose diet (HFFD) showed significant reduction in fasting blood glucose levels as compared to littermate controls (Fig. 3B, E, H, K and Suppl. Table 4 and 5). However, Slc39a5 $5^{+-}$mice did not show a similar improvement in fasting blood glucose (Fig. S2D and E), indicating that loss of one copy of Slc39a5 does not actuate a protective glucose lowering mechanism; hence, we leveraged Slc39a5/- mice for further experimentation.

Consistent with the observed glucose lowering in SLC39A5 pLOF carriers, S/c39a5 $/ /$ mice demonstrated improved glucose tolerance and insulin sensitivity in these models (Fig. 3C, F, I, L and Fig. S3). However, there were no differences in insulin 
secretion (fed and fasting) between Slc39a5\%- and littermate controls (Fig. S5E-F, S6E-F, S7E-F, S8E-F). Also, no differences were detected in insulin production or clearance in heterozygous carriers of SLC39A5 LOF variants as compared to age, sex, BMI-matched reference controls (Fig. 1D-H) upon serum call back analyses. Combined with the fact that single-cell transcriptomic data in both human and mouse show no expression of SLC39A5 in pancreatic $\beta$-cells ${ }^{7}, 8,15,16$, these results indicate that the glucose lowering effects in S/c39a5\%- mice appear to be independent of pancreatic $\beta$-cell function

\section{Loss of S/c39a5 improves liver function}

Given that NAFLD and T2D are concurrent comorbidities characterized by hepatic steatosis, glucose intolerance and insulin resistance ${ }^{17}$, we explored whether loss of Slc39a5 and consequent hepatic zinc accumulation (Fig. 2B) influenced liver function, particularly in models of congenital obesity and diet-induced obesity. S/c39a5 $5^{-/,}, \mathrm{Lepr}^{-\mathrm{L}}$ mice displayed significant reductions in hepatic lipid accumulation (Fig. 4A and Fig. S4A), hepatic triglyceride content (Fig. 4B and Fig. S4B), and in serum ALT and AST levels (biomarkers of liver damage) (Fig. 4C, D, Fig. S4C, D and Suppl. Table 4) compared to littermate Lepr/- mice. Moreover, S/c39a5/-/Lepr/- mice displayed reduced NAFLD activity score (an aggregate score of macrovesicular steatosis, hepatocellular hypertrophy and inflammation) (Fig. 4E and Fig. S4E). Consistent with reduced lipid burden, expression of hepatic fatty acid synthase expression trended lower in S/c39a5 $5^{-/ ;}$Lepr/- mice (Fig. S5BD, S6B-D). Moreover, hepatic beta-hydroxybutyrate levels were elevated in Slc39a5\%;Lepr/- mice, indicative of elevated mitochondrial $\beta$-oxidation and disposal of excess hepatic lipid resulting from leptin receptor deficiency (Fig. 4F and S4F). However, there were no significant differences in hepatic glucose-6-phosphatase expression between Slc39a5-/-:Lepr/- mice and Lepr/- mice (Fig. S5B-D, 6b-d), suggesting that the observed glucose-lowering effect is not a result of reduced hepatic glucose production in congenital obesity.

Next, we examined whether loss of S/c39a5 improves liver function in diet-induced obesity. HFFD significantly increased body weight, serum ALT and AST levels, and NAFLD activity score (Suppl. Table 5). Loss of S/c39a5 had no considerable impact on body weight in this model but resulted in marked reductions of hepatic triglyceride content 
in both sexes (Fig. 4H and S4H). However, loss of S/c39a5 resulted in sex-specific differences in most NAFLD related traits, with females benefiting more significantly compared to males, displaying significant reductions in hepatic steatosis (Fig. 4G), serum ALT (but not AST) (Fig. 4I and J), NAFLD activity score (Fig. 4K), and hepatic fatty acid synthase levels (Fig. S7B-D), and a significant elevation in hepatic beta-hydroxybutyrate

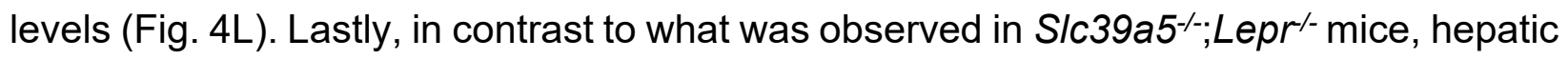
glucose-6-phosphatase levels were significantly reduced in HFFD female S/c39a5-/- mice (Fig. S7B-D), suggesting that reduced hepatic gluconeogenesis may contribute in part to the observed glucose lowering in these mice.

In HFFD male Slc39a5\%- mice, however, there were no improvements in serum ALT, AST and NAFLD activity score (Fig. S4I-K), despite reductions in hepatic triglyceride content (Fig. S4H). Significant elevation in hepatic beta-hydroxybutyrate levels and nominal reduction in hepatic fatty acid synthase levels (Fig. S4L, S8B-D) were suggestive of reduced lipid burden in HFFD male Slc39a5-/ mice. Whereas hepatic glucose-6phosphatase transcript levels were significantly reduced, protein levels showed a modest (nonsignificant) reduction suggesting that reduced hepatic gluconeogenesis may contribute to glucose lowering in HFFD male Slc39a5 significant effects observed in their female counterparts.

Taken together, these studies suggested that loss of Slc39a5 in metabolically challenged mice results in reduced hepatic lipid burden and improved hepatic insulin sensitivity leading to improved systemic glucose homeostasis.

\section{Loss of SIc39a5 results in activation of hepatic AMPK and AKT signaling}

To explore the mechanism underlying improved hepatic steatosis and glycemic traits in Slc39a5 $\%$ mice we evaluated two key signaling hubs that mediate lipid metabolism and insulin sensitivity, AMPK and AKT. Activation of hepatic AMPK signaling in a dietinduced obesity model reduces hepatic steatosis and downstream inflammation and fibrosis ${ }^{18}$, whereas activation of hepatic AKT reduces glucose production in liver ${ }^{19}$. Hence, we evaluated phosphorylation of Thr172 in AMPKa subunit (p.AMPKa) and Ser473 phosphorylation of AKT (p.AKT) in liver lysates collected at study endpoint from S/c39a5/-; Lepr/- mice and HFFD SIc39a5\%-- mice and controls. Prior to evaluating p.AMPKa and 
p.AKT, we confirmed that all Slc39a5-- mice used in these experiments displayed hepatic zinc accumulation (Fig. 5B and E, Fig. S9B and E) and increased expression of the zincresponsive genes Mt1 and Mt2 (Fig. 5C and F, Fig. S9C and F, S10B and E, S11B and E). p.AMPKa levels were elevated in both Slc39a5\%--; Lepr/- (Fig. 5A and Fig. S9A and 10A and D) and HFFD S/c39a5\%- mice (Fig. 5D and Fig. S9D and S11A and D) regardless of sex as compared to controls. However, significant hepatic AKT activation was observed only in female Slc39a5/-; Lepr/- and HFFD Slc39a5\%-/ mice (Fig. 5A and D, Fig. S9A and D, S10A and D, S11A and D).

To further explore the potential role of elevated hepatic zinc in AMPK and AKT activation, we examined whether exogenous zinc activates AMPK and AKT signaling in primary human hepatocytes. Zinc activated AKT signaling in these cells in a dose dependent manner with no adverse effect on cell viability, whereas magnesium had no effect (Fig. 5G and H, Fig. S12A). Moreover, zinc activated AMPK signaling and its downstream substrates acetyl-CoA carboxylase (ACC), and liver kinase B1 (LKB1; i.e., the kinase responsible for AMPKa Thr172 phosphorylation) (Fig. 5G and H). Timeresolved analyses of zinc-mediated activation of LKB, AMPK, and AKT indicated that zinc activates AMPK and AKT signaling acutely (within 4 hours) suggesting that zinc influences phosphorylation of these substrates independent of de novo protein synthesis (Fig. S12B). Similar results were obtained in the human hepatoma cell line HepG2 (Fig. S12C and D).

Zinc is a potent inhibitor of protein phosphatases, including protein phosphatase 2A (PP2A) and protein tyrosine phosphatase-1B (PTP1B) ${ }^{20,21}$, both of which regulate the phosphorylation of AMPKa. Liver-specific ablation of Ppp2ca (encoding PP2A's catalytic subunit) improves glucose tolerance and insulin sensitivity in mice ${ }^{22}$, whereas liverspecific ablation of Ptpn1 (encoding PTP1B) improves glucose tolerance, insulin sensitivity, and lipid metabolism ${ }^{23}$. Given that hepatic zinc is elevated in S/c39a5 $5^{-/}$mice, we evaluated hepatic phosphoserine/threonine (p.Ser/Thr) and phosphotyrosine (p.Tyr) phosphatase activity in the congenital and diet-induced obesity mice at endpoint. S/c39a5${ }^{/} ;$Lepr $^{/-}$mice displayed reduced p.Ser/Thr and p.Tyr phosphatase activity compared to Lepr/- littermate controls (Fig. S13A and B). Under HFFD, female Slc39a5 
reduced hepatic p.Ser/Thr and p.Tyr phosphatase activity by $33 \%$ and $28 \%$ respectively, and non-statistically significant reductions were also observed in male S/c39a5/- mice (Fig. S13C and D). Consistent with these observations, exogenous zinc inhibited p.Ser/Thr and p.Tyr phosphatase activity in primary human hepatocytes in a dose dependent manner (Fig. S13E). These results point to zinc mediated inhibition of protein phosphatase activity as a likely mechanism underlying hepatic AMPK and AKT activation in Slc39a5\%- mice.

\section{Loss of Slc39a5 reduces hepatic inflammation and fibrosis upon a NASH dietary challenge}

NAFLD encompasses a continuum of liver conditions from nonalcoholic fatty liver characterized by steatosis, to nonalcoholic steatohepatitis (NASH) characterized by inflammation and fibrosis ${ }^{24}$. The improvements in liver function and steatosis in congenital and diet-induced obesity mouse models lacking Slc39a5, led us to investigate whether loss of SIc39a5 protects against NASH. Diet-induced NASH significantly increased serum ALT and AST levels (Fig. 6A and B, Fig. S14A and B), body weight, fasting blood glucose (Fig. S15A, B, F, G) and liver fibrosis (Fig. 6H and I, Fig. S14H and I) in S/c39a5 $5^{+/+}$mice (Suppl. Table 6). In contrast, Slc39a5 $\%$ mice challenged with diet-induced NASH displayed significant reductions in serum ALT and AST levels (Fig. 6A and B, Fig. S14A and B) and fasting blood glucose (Fig. S15B and G), along with significant improvements in hepatic inflammation and fibrosis (Fig. 6E and H, Fig. S14E and H) and the expected increases in serum and hepatic zinc (Fig. S15C, D, H, I). Consistently, hepatic collagen deposition (Fig. 6G, Fig. S14G) were significantly reduced in NASH Slc39a5\%- mice. However, NASH Slc39a5-- mice were not protected from hepatic steatosis or hepatocyte hypertrophy (Fig. 6C and D, S14C and D). NAFLD activity score and steatosis-activityfibrosis score (sum of NAFLD activity score and fibrosis score) were significantly reduced in female NASH Slc39a5\%-- mice, but not in their male counterparts (Fig. 6F and I, Fig. S14F and I). Nonetheless, hepatic superoxide dismutase (SOD) activity was significantly elevated in both sexes in NASH Slc39a5-- mice (Fig. S15E and J), suggesting that the increase in hepatic zinc may be ameliorating the increased hepatic oxidative stress observed in $\mathrm{NASH}^{24}$. 
In aggregate, these studies suggest that the favorable metabolic profile in the Slc39a5 $\%$ mice results from convergent hepatoprotective effects of reduced lipotoxic and oxidative stress.

\section{Discussion}

Zinc is a required trace element for many biological processes. Hence, homeostatic mechanisms have evolved to maintain optimal zinc levels across tissues ${ }^{1}$. This regulation is accomplished by multiple transporters encoded by the $S L C 30$ and SLC39 gene families ${ }^{2}$. Given the apparent complexity of the system, we chose to take a human genetics approach to search for zinc transporter genes associated with metabolic traits and discovered a novel association of LOF variants in SLC39A5 with increased circulating zinc $\left(p=4.9 \times 10^{-4}\right)$ and a reduced risk of T2D $(\mathrm{OR} 0.82,95 \% \mathrm{Cl} 0.68-0.99$, $\left.p=3.7 \times 10^{-2}\right)$.

To firm up this association and explore underlying molecular mechanisms, we generated mice lacking Slc39a5. In line with the human data, and consistent with the proposed role of S/c39a5 as a non-redundant cell surface zinc transporter facilitating endogenous zinc excretion ${ }^{9,10,12}$, there was significant zinc accumulation in S/c39a5/mice across several tissues including liver (Suppl. Table 2). However, there was no significant accumulation of zinc in tissues of Slc39a5 heterozygous-null mice on a zinc adequate diet despite significant increases in serum zinc ( $26 \%$ in females and $\sim 23 \%$ in males; Fig. 2), suggesting that the relative increase of serum zinc in heterozygotes (albeit significant) is insufficient to increase zinc levels in tissues with substantial zinc stores such as the liver.

Nonetheless, given the connection with protective effects arising from heterozygous loss of SLC39A5 in humans, we examined the effect of loss of S/c39a5 in mice under conditions of metabolic stress, employing models of congenital or dietinduced obesity, and NASH. We demonstrate that in all three models, loss of S/c39a5 (in homozygosis) has protective effects that arise from elevation of circulating and hepatic zinc levels. In congenital or HFFD-induced obesity, there was improvement in glycemic traits and liver function, and a reduction of steatosis, which were not accompanied by reductions in body weight or changes in insulin secretion. In a model of diet-induced 
$\mathrm{NASH}$, loss of S/c39a5 reduced hepatic inflammation and fibrosis, but without significant changes in steatosis. Mechanistically, these protective effects result at least in part from inhibition of protein phosphatases (as result of elevated levels of zinc), and consequent increase in hepatic AMPK and AKT activation.

The observed protective metabolic effects appear to be extra-pancreatic in both mice and humans, as supported by several lines of evidence. Carriers of heterozygous LOF mutations in SLC39A5 have elevated serum zinc but exhibit no differences in insulin production or clearance as compared to age, sex, and BMI-matched homozygous reference controls (Fig. 1C-E). As in humans, loss of S/c39a5 in mice results in elevated serum zinc (Fig. 2A) without impairment in pancreatic function (Suppl. Table 3). Moreover, the observed antihyperglycemic effects in S/c39a5/- mice are not driven by changes in insulin production or clearance (Fig. S5-S8). Taken together, these observations suggest that the protective metabolic changes are extra-pancreatic.

Furthermore, our data strongly indicates that the protective effects of loss of Slc39a5 are actuated by elevated hepatic zinc concentrations. Several lines of evidence support this interpretation. First, metabolic challenges in the form of congenital or dietinduced obesity in mice revealed hepatic zinc deficiency (Fig. 5B, Fig. S9B) along with associated comorbidities including hepatic steatosis, increased fasting blood glucose and impaired insulin sensitivity (Fig. 3). Second, loss of Slc39a5 in these models resulted in the accumulation of serum zinc and hepatic zinc and concomitant improvement in liver function (Fig. 4-5, Fig. S4) and systemic glucose homeostasis (Fig. 3, Fig. S3). This data are consistent with observations that zinc deficiency is associated with obesity ${ }^{25}$ and is a biochemical hallmark of fatty liver disease in both rodents and humans ${ }^{26}$; conversely, zinc supplementation reverses manifestations of zinc deficiency in fatty liver disease and longterm oral zinc supplementation can support liver function and prevent hepatocellular carcinoma development in patients with chronic liver diseases ${ }^{27}$.

The importance of hepatic zinc in the protective effects against obesity and NASH is further supported by our findings that elevated hepatic zinc in S/c39a5/- mice enhanced hepatic AMPK and hepatic AKT signaling (Fig. 5 and Suppl Fig. 9). These increases correlated with reductions in hepatic p.Ser/Thr phosphatase and p.Tyr phosphatase levels in both diet-induced and congenital obesity models (Suppl. Fig. S13A-D), and were 
corroborated by in vitro evidence (Fig. S13E-F). These findings mirror prior studies showing that zinc inhibits protein serine/threonine and tyrosine phosphatases that dephosphorylate AMPK and AKT 20, 28-32. In turn, in states of lipotoxic stress, serine/threonine phosphatases such as PP2A and PP2C inhibit AMPK resulting in a feed forward effect of the lipid overload ${ }^{33}, 34$, whereas protein tyrosine phosphatases including PTP1B, TCPTP and PTEN have been implicated in systemic glucose homeostasis by regulating the PI3K-AKT pathway ${ }^{23,31,35,36 .}$

Overall, our studies indicate that the favorable metabolic profile observed in the S/c39a5 - mice results from the loss of endogenous zinc excretion and concomitant systemic zinc redistribution. Our study provides for the first-time genetic evidence demonstrating the protective role of zinc against hyperglycemia and unravels the mechanistic basis underlying this effect. Taken together, these observations suggest SLC39A5 inhibition as a potential therapeutic avenue for T2D, and other indications where zinc supplementation alone is inadequate.

\section{Acknowledgements:}

We wish to thank individuals that consented to be part of the Regeneron Genetics Center-Geisinger Health System DiscovEHR study. We would also like to acknowledge Kristy Neiman and William Poueymirou for their assistance with mouse colony management, and Suganthi Balasubramanian for insightful comments and input at the inception of the project, and Sergio Fazio for a critical reading of our manuscript. 
medRxiv preprint doi: https://doi.org/10.1101/2021.12.08.21267440; this version posted December 9, 2021. The copyright holder for this preprint (which was not certified by peer review) is the author/funder, who has granted medRxiv a license to display the preprint in perpetuity. All rights reserved. No reuse allowed without permission.

\section{References:}

[1] Jackson MJ, Jones DA, Edwards RH. Tissue zinc levels as an index of body zinc status. Clin Physiol 1982;2:333-343.

[2] Dempski RE. The cation selectivity of the ZIP transporters. Curr Top Membr 2012;69:221-245.

[3] Ranasinghe P, Wathurapatha WS, Galappatthy P, Katulanda P, Jayawardena R, Constantine GR. Zinc supplementation in prediabetes: A randomized double-blind placebocontrolled clinical trial. J Diabetes 2018;10:386-397.

[4] Flannick J, Thorleifsson G, Beer NL, Jacobs SB, Grarup N, Burtt NP, et al. Loss-of-function mutations in SLC30A8 protect against type 2 diabetes. Nat Genet 2014;46:357-363.

[5] Dwivedi OP, Lehtovirta M, Hastoy B, Chandra V, Krentz NAJ, Kleiner S, et al. Loss of ZnT8 function protects against diabetes by enhanced insulin secretion. Nat Genet 2019;51:15961606.

[6] Valenzuela DM, Murphy AJ, Frendewey D, Gale NW, Economides AN, Auerbach W, et al. High-throughput engineering of the mouse genome coupled with high-resolution expression analysis. Nat Biotechnol 2003;21:652-659.

[7] Baron M, Veres A, Wolock SL, Faust AL, Gaujoux R, Vetere A, et al. A Single-Cell Transcriptomic Map of the Human and Mouse Pancreas Reveals Inter- and Intra-cell Population Structure. Cell Syst 2016;3:346-360 e344.

[8] Muraro MJ, Dharmadhikari G, Grun D, Groen N, Dielen T, Jansen E, et al. A Single-Cell Transcriptome Atlas of the Human Pancreas. Cell Syst 2016;3:385-394 e383.

[9] Wang F, Kim BE, Petris MJ, Eide DJ. The mammalian Zip5 protein is a zinc transporter that localizes to the basolateral surface of polarized cells. J Biol Chem 2004;279:51433-51441.

[10] Geiser J, De Lisle RC, Andrews GK. The zinc transporter Zip5 (Slc39a5) regulates intestinal zinc excretion and protects the pancreas against zinc toxicity. PLoS One 2013;8:e82149.

[11] Wang X, Gao H, Wu W, Xie E, Yu Y, He X, et al. The zinc transporter Slc39a5 controls glucose sensing and insulin secretion in pancreatic beta-cells via Sirt1- and Pgc-1alphamediated regulation of Glut2. Protein Cell 2019;10:436-449.

[12] Dufner-Beattie J, Kuo YM, Gitschier J, Andrews GK. The adaptive response to dietary zinc in mice involves the differential cellular localization and zinc regulation of the zinc transporters ZIP4 and ZIP5. J Biol Chem 2004;279:49082-49090.

[13] Huang BW, Chiang MT, Yao HT, Chiang W. The effect of high-fat and high-fructose diets on glucose tolerance and plasma lipid and leptin levels in rats. Diabetes Obes Metab 2004;6:120-126.

[14] King AJ. The use of animal models in diabetes research. Br J Pharmacol 2012;166:877894.

[15] Tabula Muris C. A single-cell transcriptomic atlas characterizes ageing tissues in the mouse. Nature 2020;583:590-595.

[16] Xin Y, Kim J, Ni M, Wei Y, Okamoto H, Lee J, et al. Use of the Fluidigm C1 platform for RNA sequencing of single mouse pancreatic islet cells. Proc Natl Acad Sci U S A 2016;113:32933298. 
medRxiv preprint doi: https://doi.org/10.1101/2021.12.08.21267440; this version posted December 9, 2021. The copyright holder for this preprint (which was not certified by peer review) is the author/funder, who has granted medRxiv a license to display the preprint in perpetuity. All rights reserved. No reuse allowed without permission.

[17] Tilg H, Moschen AR, Roden M. NAFLD and diabetes mellitus. Nat Rev Gastroenterol Hepatol 2017;14:32-42.

[18] Garcia D, Hellberg K, Chaix A, Wallace M, Herzig S, Badur MG, et al. Genetic LiverSpecific AMPK Activation Protects against Diet-Induced Obesity and NAFLD. Cell Rep 2019;26:192-208 e196.

[19] Titchenell PM, Quinn WJ, Lu M, Chu Q, Lu W, Li C, et al. Direct Hepatocyte Insulin Signaling Is Required for Lipogenesis but Is Dispensable for the Suppression of Glucose Production. Cell Metab 2016;23:1154-1166.

[20] Bellomo E, Birla Singh K, Massarotti A, Hogstrand C, Maret W. The metal face of protein tyrosine phosphatase 1B. Coord Chem Rev 2016;327-328:70-83.

[21] Xiong $Y$, Jing $X P$, Zhou $X W$, Wang $X L$, Yang $Y$, Sun $X Y$, et al. Zinc induces protein phosphatase $2 \mathrm{~A}$ inactivation and tau hyperphosphorylation through Src dependent PP2A (tyrosine 307) phosphorylation. Neurobiol Aging 2013;34:745-756.

[22] Xian L, Hou S, Huang Z, Tang A, Shi P, Wang Q, et al. Liver-specific deletion of Ppp2calpha enhances glucose metabolism and insulin sensitivity. Aging (Albany NY) 2015;7:223232.

[23] Delibegovic M, Zimmer D, Kauffman C, Rak K, Hong EG, Cho YR, et al. Liver-specific deletion of protein-tyrosine phosphatase 1B (PTP1B) improves metabolic syndrome and attenuates diet-induced endoplasmic reticulum stress. Diabetes 2009;58:590-599.

[24] Friedman SL, Neuschwander-Tetri BA, Rinella M, Sanyal AJ. Mechanisms of NAFLD development and therapeutic strategies. Nat Med 2018;24:908-922.

[25] Marreiro DN, Fisberg M, Cozzolino SM. Zinc nutritional status in obese children and adolescents. Biol Trace Elem Res 2002;86:107-122.

[26] Mohammad MK, Zhou Z, Cave M, Barve A, McClain CJ. Zinc and liver disease. Nutr Clin Pract 2012;27:8-20.

[27] Hosui A, Kimura E, Abe S, Tanimoto T, Onishi K, Kusumoto Y, et al. Long-Term Zinc Supplementation Improves Liver Function and Decreases the Risk of Developing Hepatocellular Carcinoma. Nutrients 2018;10.

[28] Bellomo E, Abro A, Hogstrand C, Maret W, Domene C. Role of Zinc and Magnesium lons in the Modulation of Phosphoryl Transfer in Protein Tyrosine Phosphatase 1B. J Am Chem Soc 2018;140:4446-4454.

[29] Lee S, Chanoit G, McIntosh R, Zvara DA, Xu Z. Molecular mechanism underlying Akt activation in zinc-induced cardioprotection. Am J Physiol Heart Circ Physiol 2009;297:H569-575.

[30] Liangpunsakul S, Sozio MS, Shin E, Zhao Z, Xu Y, Ross RA, et al. Inhibitory effect of ethanol on AMPK phosphorylation is mediated in part through elevated ceramide levels. Am J Physiol Gastrointest Liver Physiol 2010;298:G1004-1012.

[31] Galic S, Hauser C, Kahn BB, Haj FG, Neel BG, Tonks NK, et al. Coordinated regulation of insulin signaling by the protein tyrosine phosphatases PTP1B and TCPTP. Mol Cell Biol 2005;25:819-829.

[32] Krishnan N, Konidaris KF, Gasser G, Tonks NK. A potent, selective, and orally bioavailable inhibitor of the protein-tyrosine phosphatase PTP1B improves insulin and leptin signaling in animal models. J Biol Chem 2018;293:1517-1525.

[33] Chen TC, Benjamin DI, Kuo T, Lee RA, Li ML, Mar DJ, et al. The glucocorticoid-Angptl4ceramide axis induces insulin resistance through PP2A and PKCzeta. Sci Signal 2017;10. 
[34] Wang MY, Unger RH. Role of PP2C in cardiac lipid accumulation in obese rodents and its prevention by troglitazone. Am J Physiol Endocrinol Metab 2005;288:E216-221.

[35] Pal A, Barber TM, Van de Bunt M, Rudge SA, Zhang Q, Lachlan KL, et al. PTEN mutations as a cause of constitutive insulin sensitivity and obesity. N Engl J Med 2012;367:1002-1011.

[36] Tsou RC, Bence KK. The Genetics of PTPN1 and Obesity: Insights from Mouse Models of Tissue-Specific PTP1B Deficiency. J Obes 2012;2012:926857.

\section{Figure legends:}

Fig. 1. Rare pLOF variants in SLC39A5 are associated with elevated serum zinc and nominal protection against type II diabetes (T2D). (A) Serum zinc in carriers of SLC39A5 pLOF variants in the discovery cohort. Controls (Ref; SLC39A5 ${ }^{+/+}$) and heterozygous carriers of pLOF variant alleles in SLC39A5 (Het; SLC39A5 ${ }^{+/}$). Subject numbers: Ref and Het respectively: $n=5317$ and $n=15$. (B) Trans-ancestry meta-analysis of association of SLC39A5 pLOF variants with T2D. (C-I) Serum zinc and insulin profile of age, sex and BMI-matched controls in serum call back study. Subject numbers: Ref and Het respectively: $n=246-253$ and $n=86-91,{ }^{* *} P<0.01$, unpaired t-test. Numeric data is summarized in Suppl. Table 1.

Fig. 2. Loss of SIc39a5 results in elevated circulating and hepatic zinc levels in mice. Serum zinc (A) and hepatic zinc (B) in S/c39a $5^{+/+}$, S/c39a $5^{-/-}$and S/c39a $5^{+/-}$mice at 40 weeks of age, $n=16-18$. ${ }^{* *} \mathrm{P}<0.01,{ }^{* * *} \mathrm{P}<0.001$, two-way ANOVA with post hoc Tukey's test.

Fig. 3. Loss of Slc39a5 improves glycemic traits in leptin-receptor deficient mice and in mice challenged with high fat high fructose diet (HFFD). Female (A-C, G-I; ㅇ) and Male (D-F, J-L; O) mice. (A-F) S/c39a5 ${ }^{-/} ;$Lepr $^{-/}$and corresponding control mice. (A, D) Body weight at 34 weeks. (B, E) Fasting blood glucose at 20 weeks. (C, F) Homeostatic model assessment for insulin resistance (HOMA-IR) at 34 weeks. S/c39a $5^{+/+}$and S/c39a $5^{-}$ ${ }^{\text {I- }}(\mathrm{n}=5-13)$, Lepr $^{-1-}$ and Slc39a5 ${ }^{-1} ;$ Lepr $^{-1-}(\mathrm{n}=10-15) .{ }^{*} \mathrm{P}<0.05,{ }^{* *} \mathrm{P}<0.01,{ }^{* * *} \mathrm{P}<0.001$, one-way ANOVA with post hoc Tukey's test. (G-L) S/c39a $5^{-/-}$and S/c39a $5^{+/+}$mice were fed HFFD or NC for 30 weeks. $(G, J)$ Body weight at 30 weeks. $(H, K)$ Fasting blood glucose at 18 weeks. (I, L) HOMA-IR at 30 weeks, $n=11-15$. ${ }^{*} \mathrm{P}<0.05,{ }^{* *} \mathrm{P}<0.01,{ }^{* * *} \mathrm{P}<$ 
0.001, two-way ANOVA with post hoc Tukey's test. Numeric data is summarized in Suppl. Table 4 and 5.

Fig. 4. Loss of Slc39a5 improves liver function and steatosis in leptin-receptor deficient female mice and in female mice challenged with high fat high fructose diet (HFFD). Slc39a $5^{-/-} ;$Lepr $^{-/-}$and corresponding control mice (A-F) were sacrificed after 16 hour fasting at 34 weeks of age. (G-L) S/c39a $5^{-/-}$and S/c39a $5^{+/+}$mice were fed HFFD or NC for 30 weeks and sacrificed after 16 hours fasting. (A, G) Representative images of

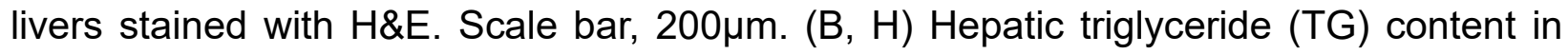
explanted liver samples at endpoint. (C, I) Serum ALT. (D, J) Serum AST. (E, K) NAFLD activity score, (F, L) Hepatic beta-hydroxybutyrate (BHOB). ${ }^{*} \mathrm{P}<0.05,{ }^{* *} \mathrm{P}<0.01,{ }^{* *} \mathrm{P}<$ 0.001, Slc39a5 $5^{-/} ;$Lepr $^{-/}$and corresponding control mice: one-way ANOVA with post hoc Tukey's test, HFFD or NC: two-way ANOVA with post hoc Tukey's test. Numeric data is summarized in Suppl. Table 4 and 5.

Fig. 5. Loss of Slc39a5 results in elevated hepatic zinc and activation of hepatic AMPK signaling in leptin-receptor deficient female mice and female mice challenged with high fat high fructose diet (HFFD). Analyses were done on explanted liver samples collected after 16 hours fasting at endpoint in Lepr- (A-C) and HFFD mice (D-F). (A, D) Immunoblot analysis of hepatic AMPK and AKT activation. AMPK and AKT signaling is activated in Lepr/-;S/c39a5 ${ }^{-/}$mice and HFFD Slc39a5\% mice (compared to

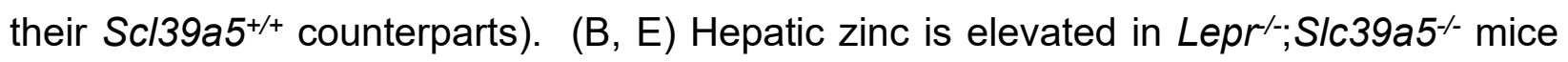
and HFFD Slc39a5-- mice $(n=10-21)$. (C, F) Elevated hepatic zinc results in increased Mt1 (zinc responsive gene) expression in both models. (G) Immunoblot analysis of primary human hepatocytes treated with zinc chloride $\left(\mathrm{ZnCl}_{2}\right)$, magnesium chloride $\left(\mathrm{MgCl}_{2}\right)$, okadaic acid (OA), metformin (Met) for 4 hours. Zinc activated AMPK and AKT signaling in primary human hepatocytes. $(\mathrm{H})$ Densitometric analysis of immunoblots (compared to control). ${ }^{*} \mathrm{P}<0.05,{ }^{* *} \mathrm{P}<0.01,{ }^{* * *} \mathrm{P}<0.001$, ANOVA with post hoc Tukey's test. 
Fig. 6. Loss of SIc39a5 improves hepatic inflammation and fibrosis in female mice challenged with diet-induced NASH. S/c39a $5^{-/-}$and S/c39a $5^{+/+}$mice were placed on a NASH inducing diet or NC for 40 weeks and sacrificed after 16 hours of fasting. (A, B) NASH SIc39a5-/- mice display reduced serum ALT and AST levels. (C-E) Histology scores for steatosis, hepatocyte hypertrophy, inflammation. (F) NAFLD activity score was reduced in NASH S/c39a $5^{-/}$mice. (G-I) NASH S/c39a5 $5^{-/}$mice display reduced fibrosis. (G) Representative images of explanted livers sample stained with picrosirius red indicative of collagen deposition. Scale bar, $300 \mu \mathrm{m}$. $(\mathrm{H}, \mathrm{I})$ Fibrosis and steatosis-activityfibrosis scores. $\mathrm{n}=6-7$ (NC) and 8-11 (NASH), ${ }^{*} \mathrm{P}<0.05$, ${ }^{* *} \mathrm{P}<0.01$, ${ }^{* * *} \mathrm{P}<0.001$, twoway ANOVA with post hoc Tukey's test. Numeric data is summarized in Suppl. Table 6. 


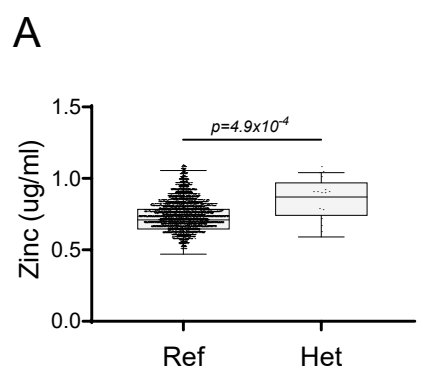

B

\begin{tabular}{|c|c|c|c|c|c|c|}
\hline STUDY & N CASES & N CONTROLS & & $\mathrm{OR}$ 105\% $\mathrm{Cl}$ & PVALUE & $\triangle A F$ \\
\hline GHS_EUR & $26686|72| 0$ & $64587|261| 0$ & $=$ & $0.663[0.5,0.878]$ & 0.0042 & 0.00182 \\
\hline MALMO_EUR & $3797|7| 0$ & $21089|50| 0$ & $\rightarrow$ & $0.827[0.39,1.752]$ & 0.62 & 0.00114 \\
\hline SINAI_AFR & $2976|4| 0$ & $5265|9| 0$ & $\longrightarrow$ & $1.403[0.331,5.952]$ & 0.65 & 0.00079 \\
\hline SINAI_AMR & $1071|5| 0$ & $1809|3| 0$ & $\Longrightarrow$ & $3.123[0.475,20.517]$ & 0.24 & 0.00138 \\
\hline SINAI_EUR & $978 \mid 110$ & $7484|14| 0$ & $=$ & $0.884[0.116,6.737]$ & 0.9 & 0.00088 \\
\hline UKB_AFR & $1085|1| 0$ & $7812|9| 0$ & $\longrightarrow$ & $0.81[0.112,5.888]$ & 0.84 & 0.00056 \\
\hline UKB_EUR & $23853|54| 0$ & $402001|927| 0$ & 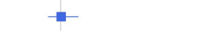 & $1.01[0.762,1.338]$ & 0.95 & 0.00115 \\
\hline UKB_SAS & $1935|1| 0$ & $8185|15| 0$ & 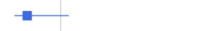 & $0.341[0.101,1.152]$ & 0.083 & 0.00079 \\
\hline \multirow[t]{2}{*}{ Meta } & $62381|145| 0$ & $518232|1288| 0$ & 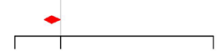 & $0.82[0.681,0.988]$ & 0.037 & 0.00123 \\
\hline & & & $0.1 \quad 1$ & & & \\
\hline
\end{tabular}

C

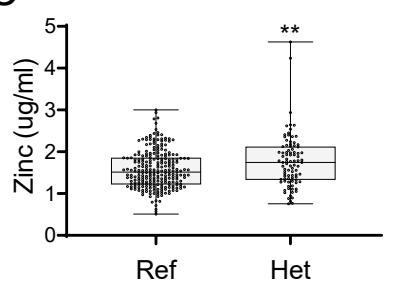

G

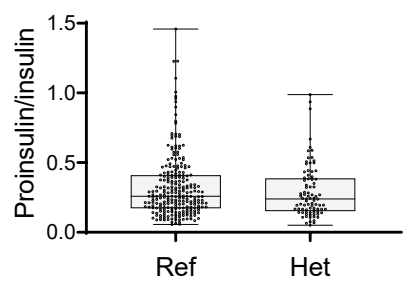

D

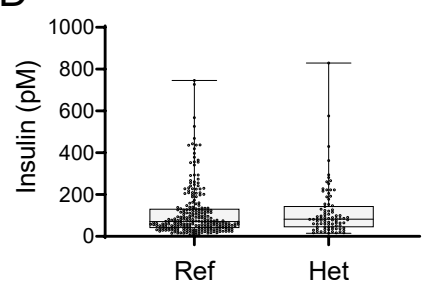

$\mathrm{H}$

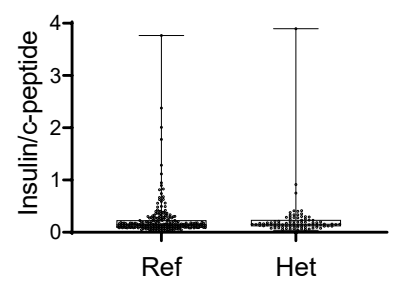

E

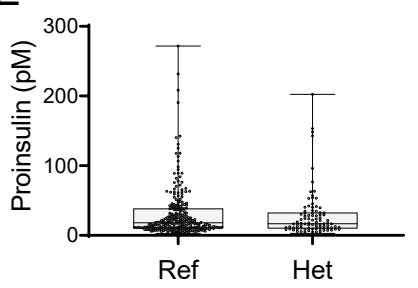

I

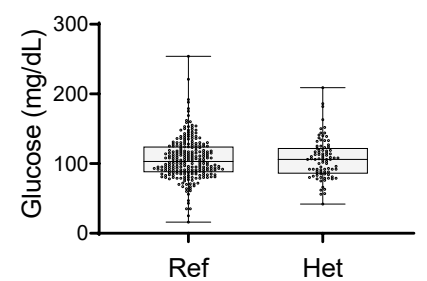

F

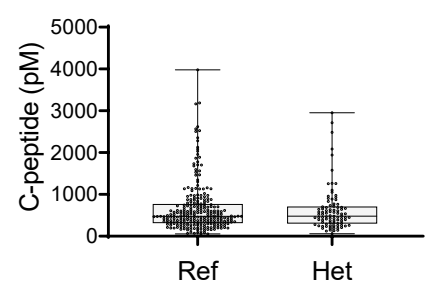

Fig. 1 
A

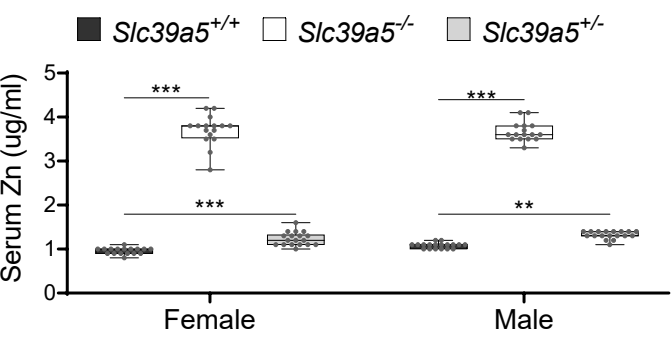

B

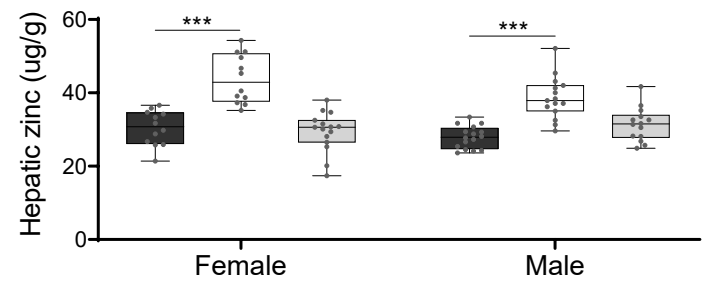

Fig. 2 
A

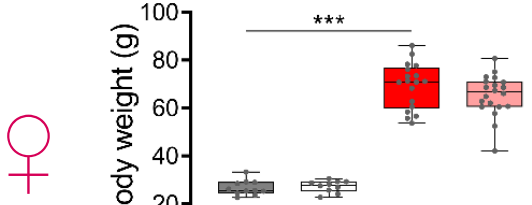

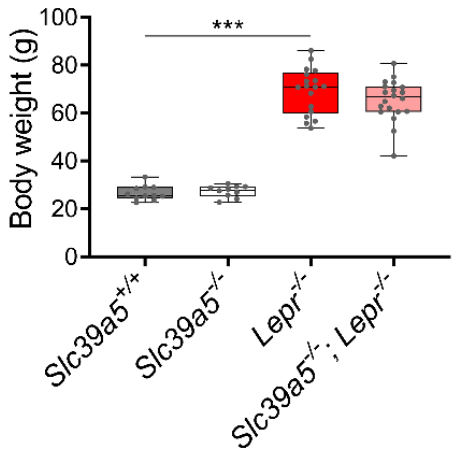

D

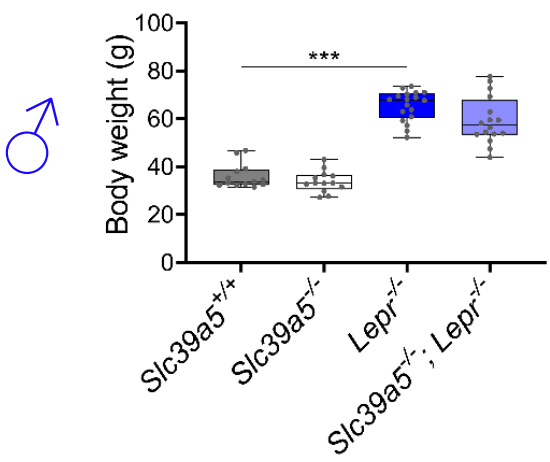

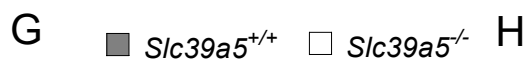

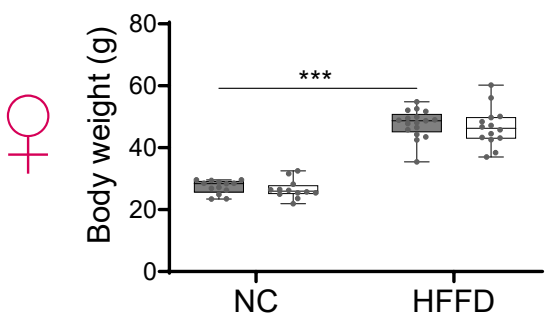

J

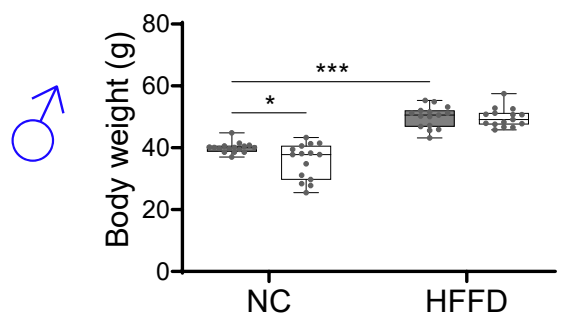

B

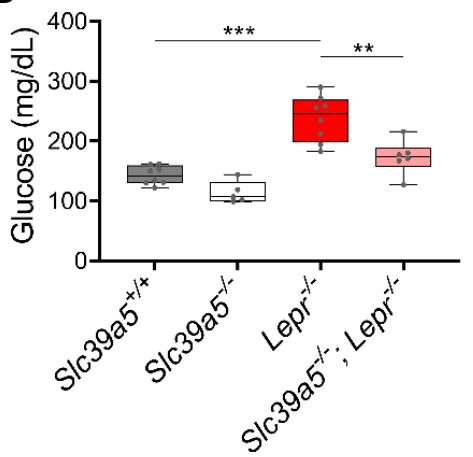

E

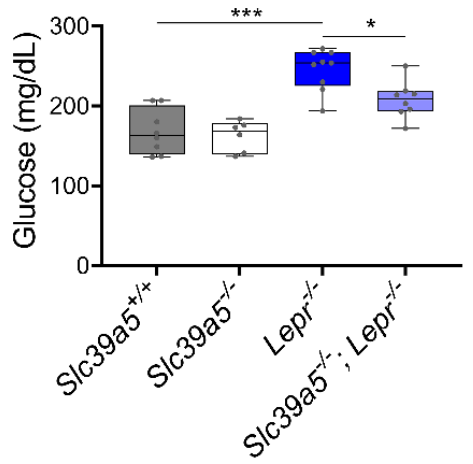

C

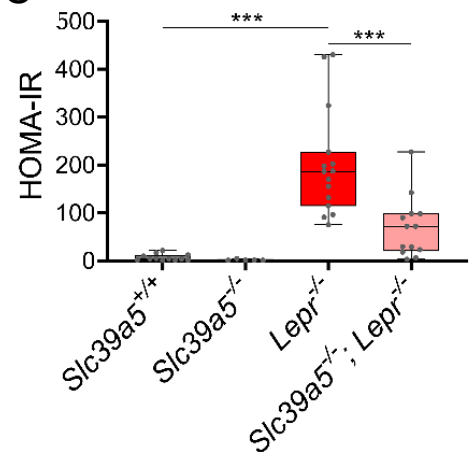

F

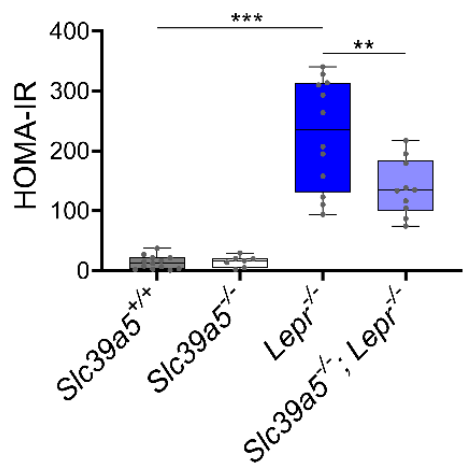

I

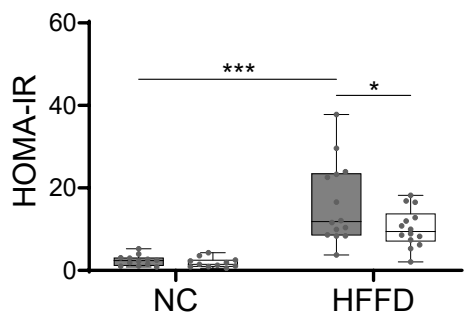

L
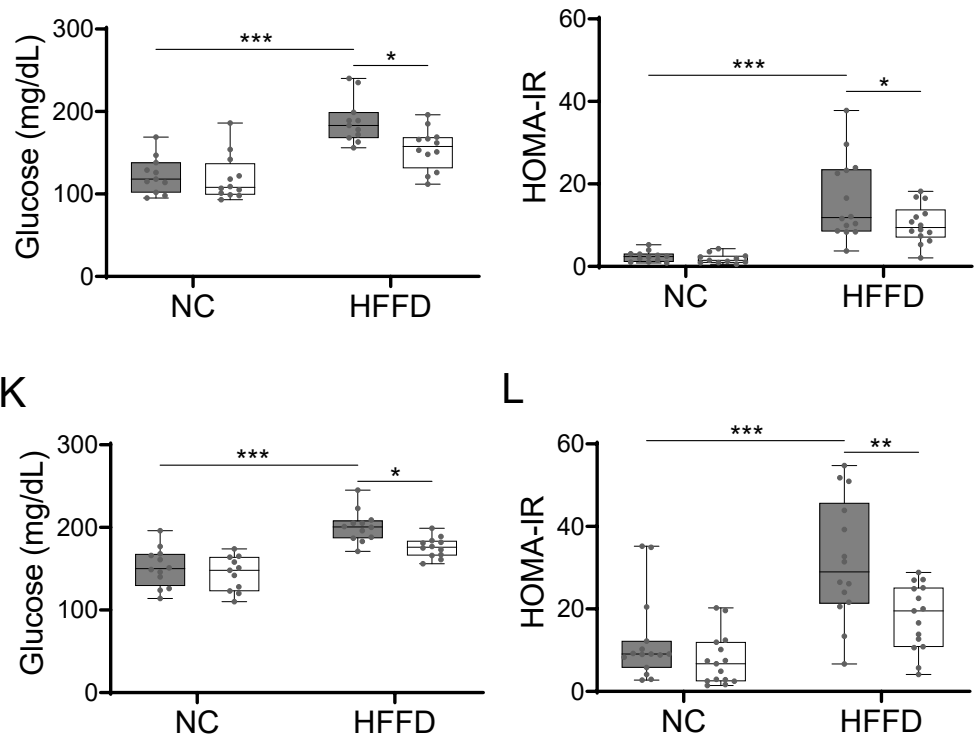

Fig. 3 
A

Slc39a $5^{+/+}$

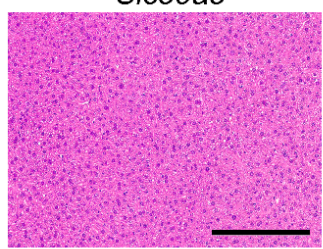

Slc39a $5^{-1}$
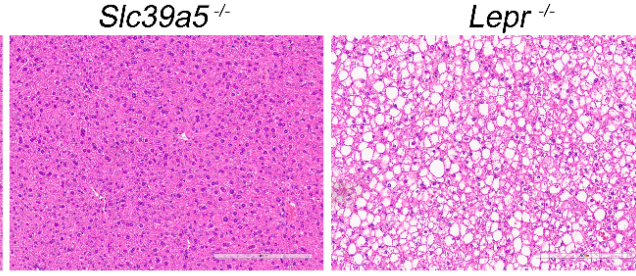

Slc39a5 $\%$ Lepr

C
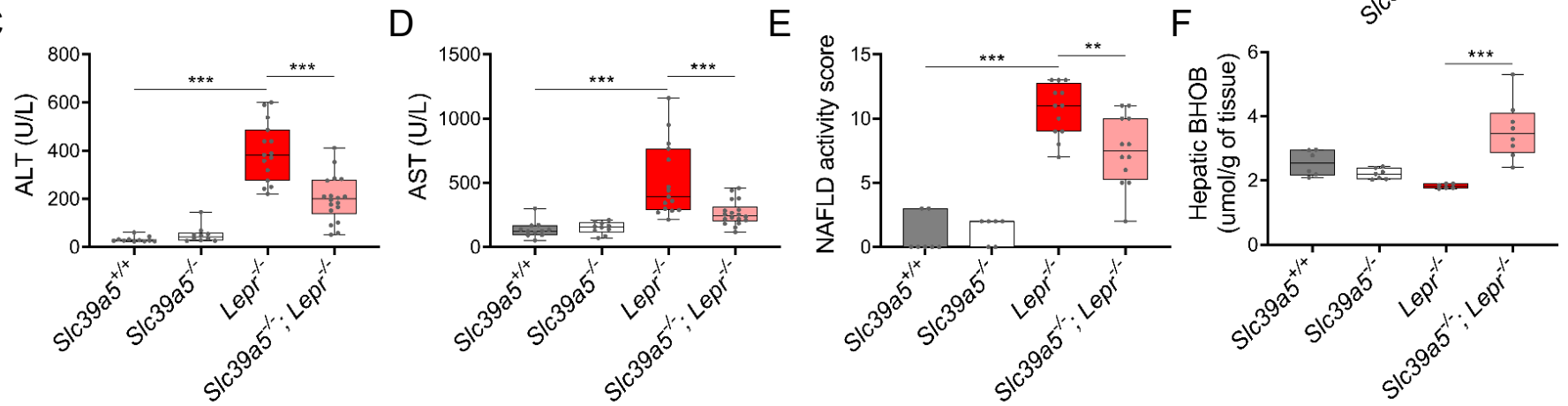

G

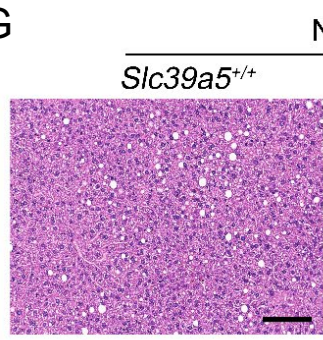

NC S1c39a5

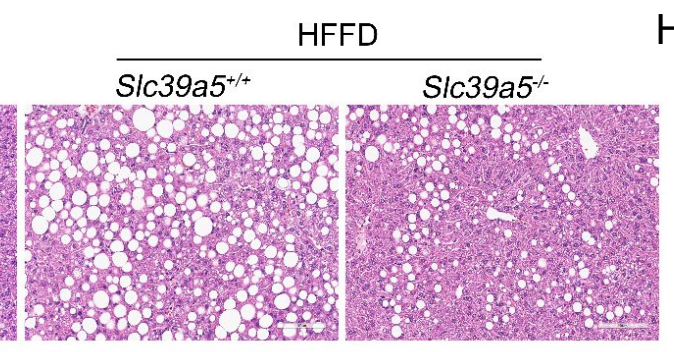

F

B

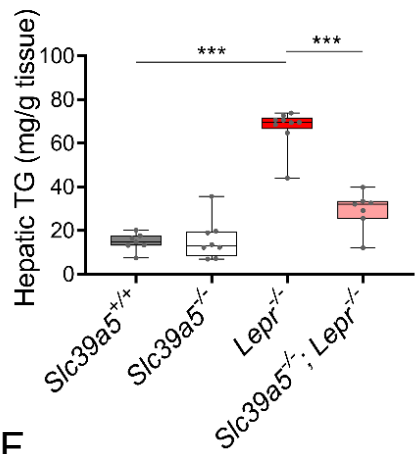

$\mathrm{H}$

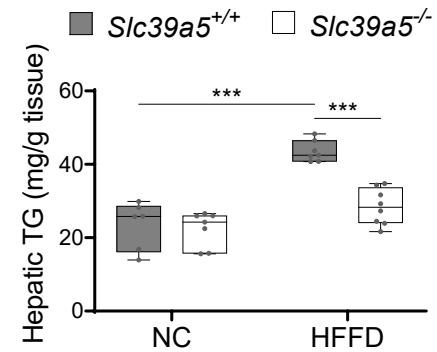

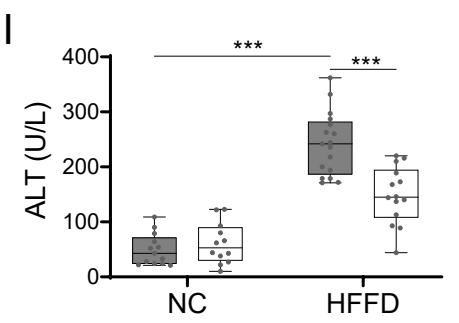
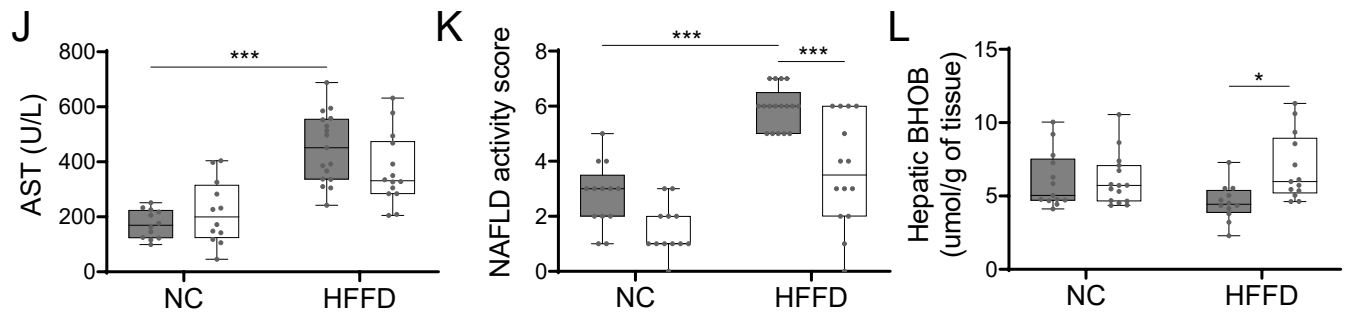

Fig. 4 
A

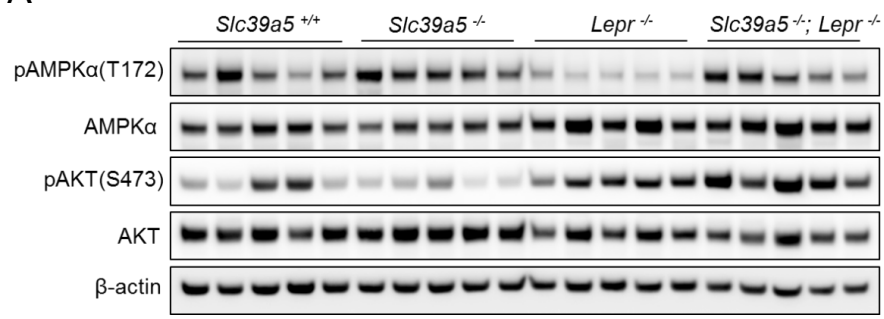

B

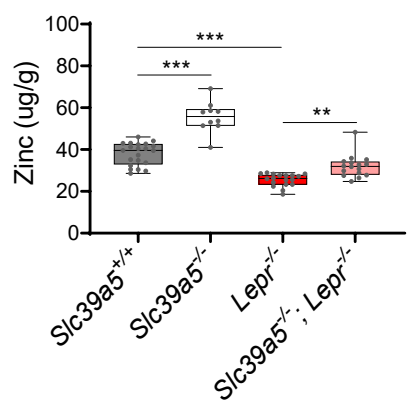

C

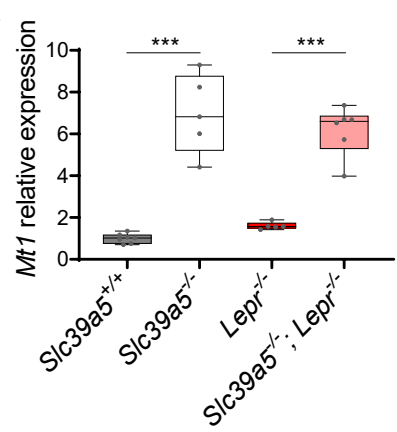

F
D

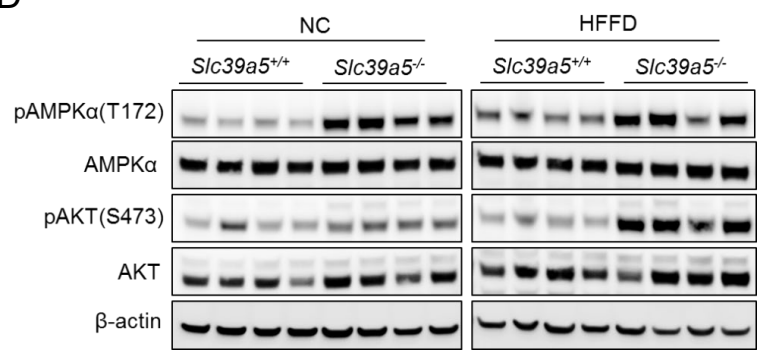

E

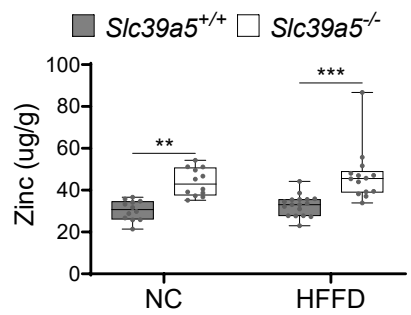

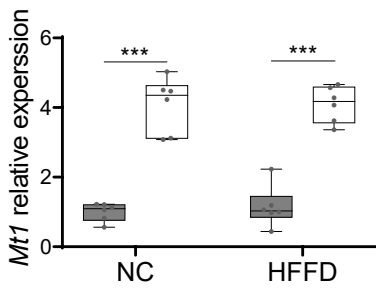

G

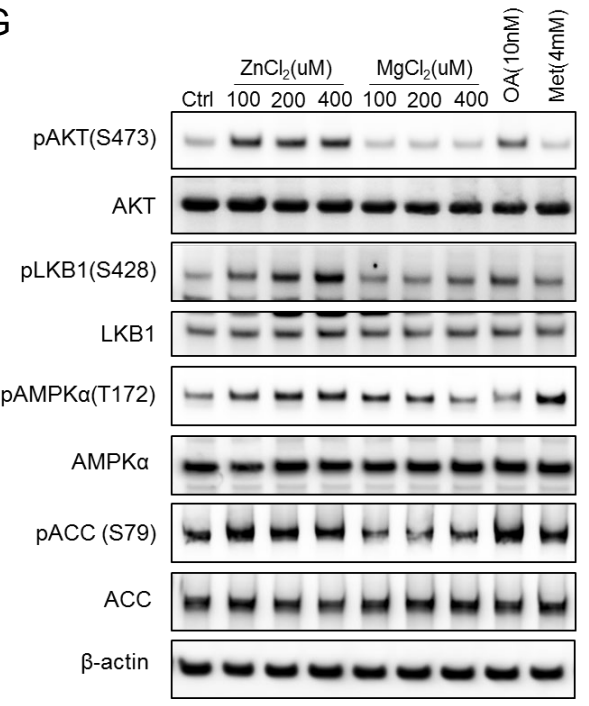

$\mathrm{H}$
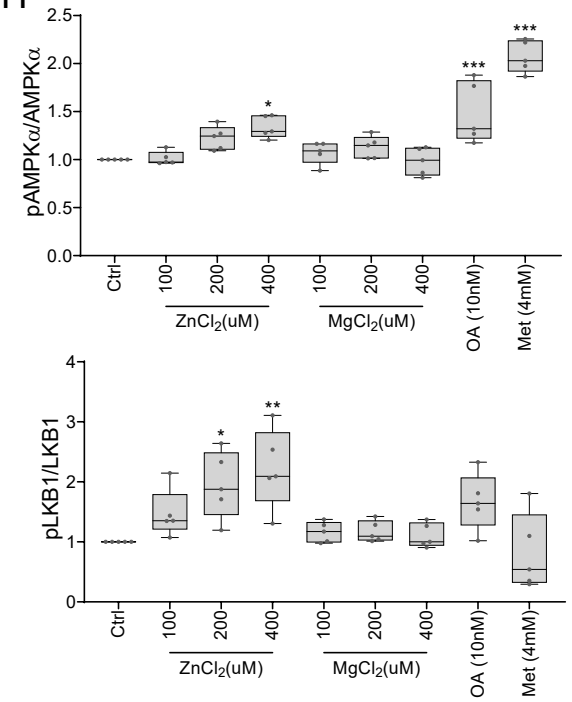
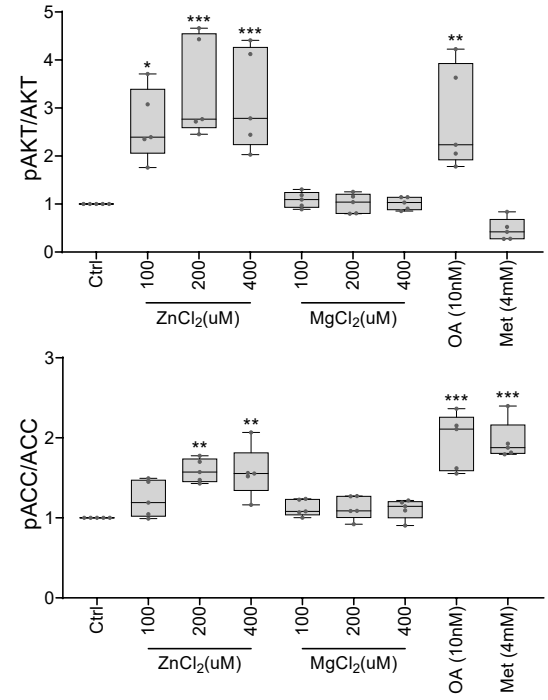

Fig. 5 
A $\square \operatorname{slc} 39 a 5^{+/+} \square S / c 39 a 5^{-/-} B$
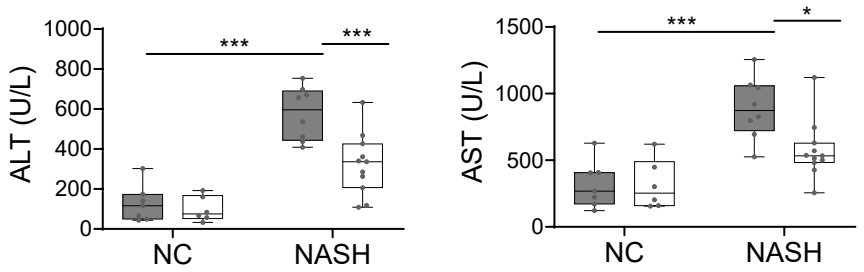

C
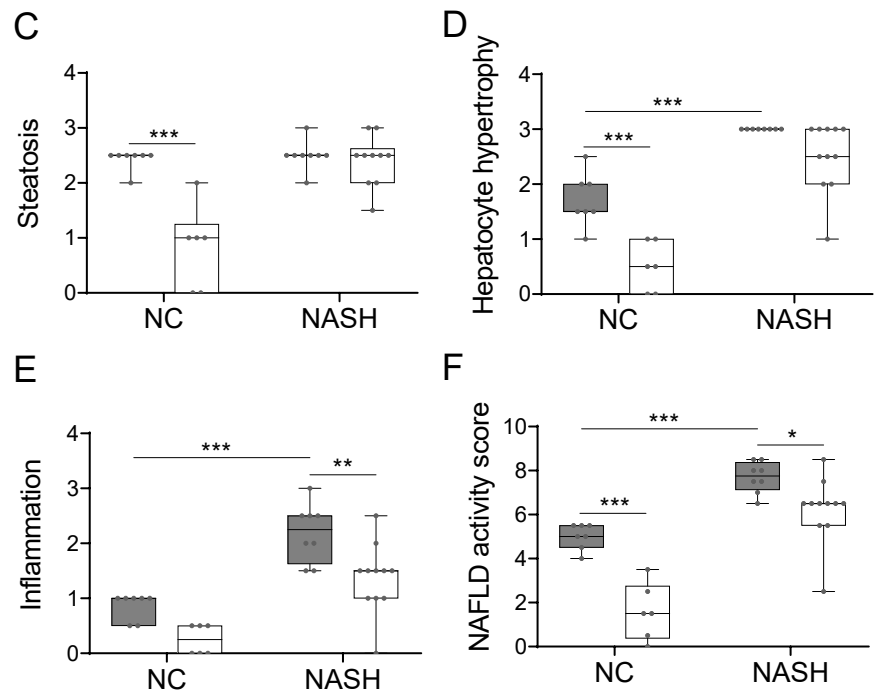

G
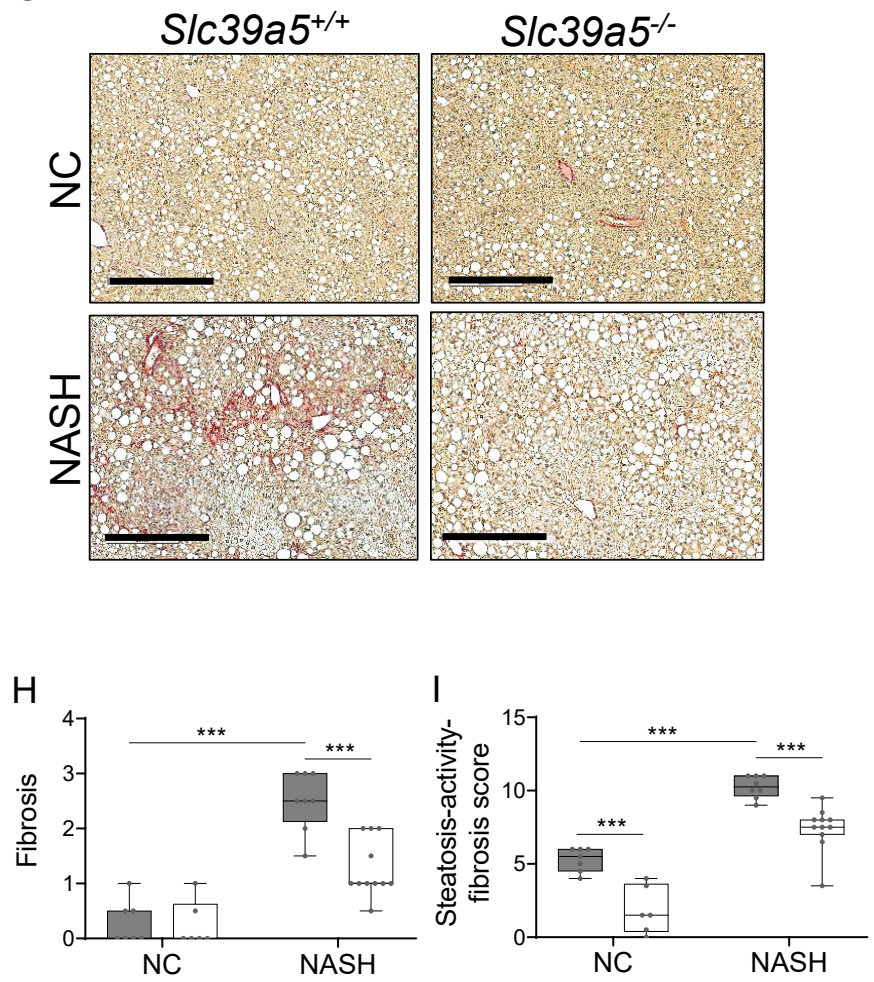

Fig.6 


\section{Supplementary Methods}

\section{Human Genetic Studies and Phenotyping}

The Geisinger Health System DiscovEHR study is a hospital-based cohort of patients of the GHS, a large healthcare provision network in Central and Eastern Pennsylvania, United States. More than 200,000 health system participants have been enrolled and $>145,000$ have had exome sequencing performed by Regeneron Genetics Center. Type 2 diabetes (T2D) cases in DiscovEHR were defined as individuals with an ICD9 (code 250) or ICD10 (code E11) code for T2D, and either a median HbA1c value greater than or equal to $6.5 \%$, or with a prescription for any diabetic medication. Individuals were excluded from the case pool if they had both an ICD10 code for type 1 diabetes (T1D; code E10) and if they did not have a prescription for any oral hypoglycemic medication. Controls were defined as individuals with no ICD codes for T1D or T2D, a median HbA1C value of less than $5.7 \%$, and with no record of a prescription for any diabetic medication.

The UK Biobank is a prospective biomedical study of $\sim 500,00$ adults from across the UK, including extensive phenotype measures and genomic data. T2D in UK Biobank was defined in line with a previously reported definition in this cohort ${ }^{1,2}$. The UKB self-reported data were used to identify individuals with "probable type 2 diabetes", "possible type 2 diabetes", "probable type 1 diabetes" or "possible type 1 diabetes", using a previously published algorithm ${ }^{1}$. T2D cases were defined as individuals with "probable type 2 diabetes" on self-report, or an ICD10 code E11 for T2D. Individuals were excluded from the analysis if they had "probable type 1 diabetes", "possible type 1 diabetes", or ICD10 code E10, for T1D.

The BioMe study (SINAI) is a highly diverse electronic health record (EHR)-linked biobank of over 50,000 participants from the Mount Sinai Health System (MSHS) in New York, NY. T2D cases in BioMe were defined as individuals meeting at least two of the following three criteria: 1) ICD10 code for T2D (code E11 and/or O24.1), 2) a blood value in keeping with diabetes (median $\mathrm{HbA} 1 \mathrm{c}$ value greater than or equal to $6.5 \%$ and/or median random glucose greater than or equal to $200 \mathrm{mg} / \mathrm{dL}$ ), and 3) a prescription for any diabetic medication. Individuals were excluded from the case pool if they had an ICD10 code for 
T1D (code E10 and/or O24.0) or if they had a record of having received an outpatient prescription for insulin (and no record of other antidiabetic medication). Controls were defined as individuals with no ICD10 codes for any type of diabetes mellitus or a family history of diabetes, median $\mathrm{HbA} 1 \mathrm{c}$ value of less than $5.7 \%$, median random glucose of less than $200 \mathrm{mg} / \mathrm{dL}$, no oral glucose tolerance test in pregnancy exceeding a diagnostic threshold for gestational diabetes, and no record of a prescription for any diabetic medication.

The Malmö Diet and Cancer Study (MDCS) is a prospective study of $\sim 53,000$ adults living in Malmö, Sweden ${ }^{3}$. T2D cases in MDCS were defined as individuals meeting at least two of the following four criteria: 1) ICD10 code for T2D (code E11 and/or O24.1) or T2D noted in diabetes registries, 2) a blood value in keeping with diabetes ( $\mathrm{HbA} 1 \mathrm{c}$ value greater than or equal to $6.5 \%$ and/or fasting glucose greater than or equal to $126 \mathrm{mg} / \mathrm{dL}$ ), 3) a prescription for non-insulin diabetic medication, and 4) a record of a non-specific diabetes event (e.g. reported at baseline, or extracted from a registry) with an age at diagnosis, or start of treatment, of greater than or equal to 35 years. Individuals were excluded from the case pool if they had an ICD10 code for T1D (code E10 and/or O24.0), or T1D noted in a diabetes registry, or if they had a record of having received insulin with no record of other antidiabetic medication. Controls were defined as individuals with no ICD10 codes for any type of diabetes mellitus, no family history of diabetes, no other variables indicating a potential diagnosis of diabetes, $\mathrm{HbA} 1 \mathrm{c}$ value of less than $5.7 \%$, fasting glucose of less than $100 \mathrm{mg} / \mathrm{dL}$, and no record of a prescription for any diabetic medication.

\section{Association analyses}

Rank-based inverse normal transformed (RINT) quantitative measures (including all subjects and sex-stratified models) with non-missing phenotype information were assessed using an additive mixed model implemented in REGENIE v2 ${ }^{4}$. Prior to normalization, traits were adjusted for a standard set of covariates including age, age ${ }^{2}$, sex, agexsex, age ${ }^{2} \times$ sex, 10 common variant genetic principal components and 20 genetic principal components derived from rare variants. Binary outcomes were similarly 
adjusted for age, age ${ }^{2}$, sex, agexsex, age ${ }^{2} \times$ sex, 10 common variant genetic principal components and 20 genetic principal components derived from rare variants and tested for association using a generalized mixed model implemented in REGENIE v2. Following analysis within each cohort, we performed inverse variance-weighted meta-analysis for T2D using METAL.

\section{GHS serum Call Back Study}

As an orthogonal biochemical assessment of the EHS reported blood analyte data, a serum callback study was designed to evaluate serum zinc, blood glucose, insulin synthesis (proinsulin/insulin ratio) and clearance (insulin/c-peptide ratio) in heterozygous carriers of SLC39A5 pLOF variants. Carriers of pLOF variants in SLC39A5 among exome-sequenced participants of European ancestry in the Regeneron Genetics CenterGeisinger Health System DiscovEHR study were included. Controls included non-carriers of pLOF variants in SLC39A5 and SLC30A8 or the common T2D risk variant rs13266634 in SLC30A8 and non-carrier first degree relatives of study subjects. Participants with T1D or T2D diagnoses were excluded. Furthermore, two non-carriers were selected for each carrier matching sex, age (+/-5years) and BMI (+/-5). A total of 22 SLC39A5 LOF variants in 131 carriers and 262 matched non-carriers were identified, however sample (frozen fasting serum) availability limited analyses to $\sim 250$ non-carriers and $\sim 90$ carriers as shown in Supplementary Table 1. Serum insulin was measured using Human Insulin ELISA kit (Millipore, EZHI-14BK), proinsulin using Human Total Proinsulin ELISA kit (Millipore, EZHPI-15BK), and c-peptide using Human c-peptide ELISA kit (Abcam, ab178611). Serum zinc was measures using flame atomic absorption spectroscopy as described below. Blood glucose was evaluated using ADVIA Chemistry Glucose Hexokinase_3 reagents (REF 050011429) on a Siemens ADVIA Chemistry XPT analyzer.

\section{Generation of Slc39a5 loss of function mice}

The genetically engineered $S / c 39 a 5^{-1-}$ mouse strain was created using Regeneron's VelociGene ${ }^{\circledR}$ technology ${ }^{5}, 6$. Briefly, C57BI/6NTac embryonic stem cells (ESC) were targeted for ablation of a portion of S/c39a5, beginning just after the initiating 
ATG and ending 5 base pairs before the 3' end of coding exon 2. This region contains the SLC39A5 signal peptide and much of the N-terminal extracellular domain. A lacZ reporter module was inserted in frame with Slc39a5's initiating Methionine codon, followed by a self-deleting fLoxed neomycin resistance (neo) cassette for selection in mouse C57BL/6NTac embryonic stem cells. The targeted cells were microinjected into 8cell embryos from Charles River Laboratories Swiss Webster albino mice, yielding F0 VelociMice $^{\circledR}$ that were $100 \%$ derived from the targeted cells ${ }^{5}$. These mice were subsequently bred to $\mathrm{F} 1$, at which point the self deleting neo cassette was also removed in the male germline. F1 heterozygotes were utilized to generate experimental cohorts, including Slc39a5 ${ }^{-/+}$heterozygous mice and wild-type littermates that were used as controls; this line was maintained in Regeneron's animal facility in the C57BI/6NTac genetic background throughout the study.

\section{Animal studies}

Mice homozygous for S/c39a5 loss of function and wild-type littermates were cohoused in a controlled environment (12hr light/dark cycle, $22 \pm 1^{\circ} \mathrm{C}, 60-70 \%$ humidity) and fed ad-libitum. All studies were performed in both sexes. For HFFD study, ten-weekold mice were fed HFFD diet (46kcal\% Fat, 30kcal\% Fructose, TestDiet 5WK9) or control diet (TestDiet 58Y2) for 30 weeks. For NASH study, ten-week-old mice were fed NASH diet (40kcal\% Fat, $20 \mathrm{kcal} \%$ Fructose and 2\% Cholesterol, ResearchDiets D09100310) or control diet (ResearchDiets D09100304) for 40 weeks. Both HFFD and NASH diets contain $\sim 34 \mathrm{ppm}$ zinc as described in diet spec sheets, and further confirmed by flame atomic absorption spectrometry. S/c39a $5^{-/-} ;$Lepr $^{/-}$mice and corresponding control mice $\left(\right.$ Slc39a5 $5^{+/+} ;$Lepr $^{/-}$, Slc39a5 $5^{-/-} ;$Lepr $^{+/+}$, and Slc39a5 $5^{+/+} ;$Lepr $^{+/+}$mice) were fed a normal chow (LabDiet 5053, containing 87ppm zinc) for 34 weeks. All mice used in this study were housed in pathogen-free environment at Regeneron Pharmaceuticals Inc. animal research facility. Sterile water and show were given ad libitum.

\section{Ethics Statement}

All experimental protocols in mice including anesthesia and tissue sampling procedures performed in this study, were approved by Regeneron Pharmaceuticals Inc. 
Institutional Animal Care and Use Committee (IACUC) under protocol number 430 and the US Animal Welfare Act.

\section{Serum Analysis}

Sera were collected upon an overnight fast (16 hours). The liver and lipid profile were analyzed using Siemens ADVIA Chemistry XPT analyzer which is maintained and operated according to Siemens' guidelines. The liver and lipid profile contains the following reagents: Alanine Aminotransferase (ALT, Siemens REF 03036926), Aspartate Aminotransferase (AST, Siemens REF 07499718), Cholesterol (CHOL, Siemens REF 10376501), Direct HDL Cholesterol (DHDL, Siemens REF 07511947), LDL Cholesterol Direct (DLDL, Siemens REF 09793248), Non-Esterified Fatty Acids (NEFA, Wako 99934691, 995-34791, 991-34891, 993-35191), Triglycerides (TRIG, Siemens REF 10335892. When mixed with sample, reagents undergo a colorimetric change proportional to the concentration of the specific analyte. The absorbance is then measured with a halogen light source and used to determine concentration. Serum was also collected for ELISA analysis of proinsulin (Mercodia, \#10-1232-01) per manufacturer's guidelines. Briefly, samples were incubated with enzyme conjugate at room temperature for two hours and washed. Substrate TMB was added and the reaction was allowed to proceed for 30 minutes at room temperature before stop solution was applied. Optical density was read at $450 \mathrm{~nm}$. Luminex Metabolic panel serum analyses of insulin, c-peptide were performed using a Mouse Metabolic Hormone Magnetic Bead Panel (Millipore, MMHMAG-44K). Experimental protocols for the sample collection, storage, preparation of reagents for immunoassay and immunoassay procedure, followed the specific instructions of the MMHMAG-44K mouse panel supplier. Results were read on a Luminex 200 analyzer with XX software (Xponent/Analyst version 4.2) used for data analysis. Insulin profile was also analyzed in serum collected from mice at fed state.

\section{Metal ion quantification}

Assays were performed by the Louisiana Animal Disease Diagnostics Laboratory with an Agilent Technologies 240 FS Atomic Absorption Spectrometer, in flame mode. Serum samples are quantitatively diluted in deionized water and subsequently analyzed. 
For the serum samples a Seronorm Trace Elements Serum (L-2) is used as reference. First tissue samples are weighed and digested in nitric acid overnight at $85^{\circ} \mathrm{C}$. The following day, the samples are cooled down to room temperature and quantitatively transferred to polystyrene tubes with deionized water, and subsequently analyzed. For all tissue samples, a bovine liver standard reference material (SRM 1577c) from the National Institute of Standards and Technology was used as reference.

\section{Liver histology and histopathologic analysis}

Explanted liver samples were fixed in $10 \%$ phosphate buffered formalin acetate at $4^{\circ} \mathrm{C}$ overnight, thoroughly rinsed in phosphate-buffered saline and transferred to $70 \%$ ethanol. Histology was performed by HistoWiz Inc. and Histoserv Inc. using standard operating procedures and fully automated workflow. Samples were embedded in paraffin wax and sectioned (5 $\mu \mathrm{m})$. Prior to staining, slides were deparaffinized in xylene and hydrated with graded alcohols and finally water. Slides were then stained with either hematoxylin \& eosin (H\&E) or Picrosirius Red. Immunohistochemistry was performed on a Bond Rx autostainer (Leica Biosystems) with heat-induced epitope retrieval. Slides were incubated with primary antibodies F4/80 (Thermo, \#14-4801-82), a-smooth muscle actin (Abcam, \#ab5694) and Bond Polymer Refine Detection (Leica Biosystems) was used per manufacturer's protocol. Following staining, slides were dehydrated and coverslipped using a TissueTek-Prisma and Coverslipper (Sakura). Whole slide scanning (40x) was performed on an Aperio AT2 (Leica Biosystems). For lipid staining, samples were frozen in O.C.T. compound (Tissue-Tek, \#4583) and $5 \mu \mathrm{m}$ thick sections were used. Slides were stained with Oil Red O (ORO) and Mayers hematoxylin and mounted with glycerin jelly. NAFLD scoring was performed by one external pathologist (provided by Histowiz) and one internal pathologist blinded to the samples, according to criteria described by Liang et $\mathrm{al}^{7}$. Macrovesicular steatosis (H\&E, ORO), hepatocyte hypertrophy (H\&E), inflammation (H\&E, F4/80) and fibrosis (PSR) were scored ranging from 0 to 3. NAFLD activity score is the sum of steatosis, hepatocyte hypertrophy and inflammation scores. Steatosis-activity-fibrosis score is the sum of NAFLD activity score and fibrosis score. 


\section{Hepatic Triglyceride assay}

Lipids were extracted from liver samples using Folch method $^{8}$ and solubilized as described earlier ${ }^{9}$. The levels of triglyceride were measured using Pointe triglyceride (GPO) reagent set (MedTest Dx, \#T7532) and normalized to wet tissue weight.

\section{Glucose and insulin tolerance tests}

An oral glucose tolerance test was administered upon an overnight fast (16 hours) with free access to water. Dextrose (Hospira Inc, NDC 0409-4902-34) was administered by oral gavage per $2 \mathrm{~g} / \mathrm{kg}$ of body weight. Blood glucose was evaluated at defined time points $(0,15,30,60$ and 120 minutes) using AlphaTrak blood glucose monitoring system (Zoetis United States, Parsippany NJ) by sampling blood from the lateral tail vein. Insulin tolerance tests were performed after a 4 hour fast by administering $1.0 \mathrm{U} / \mathrm{kg}$ of body weight of Humulin R (Eli Lilly, \#HI-213) by intra-peritoneal injection. Blood glucose was again evaluated at defined timepoints with the AlphaTrak blood glucose monitoring system by sampling blood from the lateral tail vein.

\section{HOMA-IR}

Homeostatic model assessment of insulin resistance (HOMA-IR) indicates level of insulin sensitivity by taking into account the relationship between glucose and insulin. HOMA-IR was calculated according to the formula: fasting insulin (microU/L) $x$ fasting glucose $(\mathrm{nmol} / \mathrm{L}) / 22.5$.

\section{Immunoblotting}

For biochemical analysis, liver samples were harvested and immediately snap frozen in liquid nitrogen. Protein was later extracted using RIPA buffer (Cell signaling technology, \#9806) with Halt Protease \& Phosphatase Inhibitor Cocktail (ThermoFisher Scientific, \#78440). Protein concentration was determined using Pierce TM BCA protein assay kit (Thermo Scientific, \#23225). Five micrograms of protein of each sample were 
separated in NuPAGE 4-12\% Bis-Tris protein gel (Invitrogen, \#WG1403BOX), and transferred to nitrocellulose membrane using Trans-Blot@ Turbo ${ }^{\mathrm{TM}}$ Transfer System (BioRad). The membranes were blocked with 5\% non-fat dry milk (BioRad, \#9999) for 1 hour at room temperature before incubated with primary antibody overnight at $4^{\circ} \mathrm{C}$. Antibodies were purchased from Cell Signaling Technology, phospho-AKT (Ser473, \#4060), AKT (\#9272), phospho-AMPKa (Thr172, \#2535), AMPKa (\#5831), phosphoLKB1 (Ser428, \#3482), LKB1 (\#3047), phospho-ACC (Ser79, \#3661), ACC (\#3676), FASN (\#3189), HRP-linked anti-rabbit IgG (\#7074) and HRP-linked anti-mouse IgG (\#7076). Antibodies for G6PC (Invitrogen, \#PA5-42541), SLC39A5 and $\beta$-actin (Sigma \#5441) were used. For detection of SLC39A5 protein, liver samples were immunoprecipitated using Pierce Protein A/G Magnetic Beads and anti-SLC39A5 antibodies (Invitrogen, \#88803, 42522), and eluted for western blot analysis. All membranes were washed before incubation with HRP-linked secondary antibody for one hour at room temperature. Blots were developed using SuperSignal West Femto Substrate (ThermoFisher Scientific, \#34095). Signals were captured using G:Box Mini 9 (Syngene). Densitometry analysis of immunoblots were performed using ImageJ.

\section{Gene expression analysis}

Tissues were preserved in RNAlater solution immediately following harvest. Total RNA was purified using MagMAX ${ }^{\mathrm{TM}}-96$ for Microarrays Total RNA Isolation Kit (Invitrogen, \#AM1839) according to manufacturer's specifications. Genomic DNA was removed using MagMAX ${ }^{\mathrm{TM}}$ Turbo ${ }^{\mathrm{TM}}$ DNase Buffer and TURBO DNase from the MagMAX kit listed above. mRNA (Up to 2.5ug) was reverse-transcribed into cDNA using SuperScript ${ }^{\circledR}$ VILO ${ }^{\text {TM }}$ Master Mix (Invitrogen, \#11755500). cDNA was diluted to 0.55ng/uL. 2.5-25ng cDNA input was amplified with the SensiFAST Hi-ROX MasterMix (BIOLINE, \#CSA-01113) using the ABI 7900HT Sequence Detection System (Applied Biosystems). The sequences of primers are as follows: Slc39a5 ( $F$ 5'CGAGCCTAGACCTCTTCCA- 3', R 5'-GGGAGCCATTCAGACAATCC-3'), Mt1 (F 5'CAAGTGCACCTCCTGCAAGAAG-3', R 5'-CACAGCCCTGGGCACATTT-3'), Mt2 (F 5'GACCCCAACTGCTCCTGTG-3', R 5-'CTTGCAGGAAGTACATTTGCATTG-3'), G6pc (F 
5'-GGTCGTGGCTGGAGTCTTG-3', R 5'-CCGGAGGCTGGCATTGTAG-3') and Fasn (Thermofisher Scientific \#Mm00662319_m1).

\section{Human primary hepatocyte culture}

Human Plateable Hepatocytes were purchased from Invitrogen (\#HMCPP5, Lot No HPP1881027 and HPP1878738) and used according to manufacturer protocol. These Hepatocytes are a pooled population of primary hepatocytes produced by combining cells from 5 individual donors. All reagents and materials were purchased from Invitrogen. Briefly, cryopreserved hepatocytes were thawed in hepatocyte thawing medium (\#CM7500). Hepatocytes were centrifuged and resuspend in plating medium, Williams' Medium E (\#A1217601) with hepatocyte plating supplement (\#CM3000). Hepatocytes were directly plated in collagen I coated 24-well plate (\#A1142802). After 6 hours incubation, media were replaced with incubation medium, Williams' Medium E with hepatocyte maintenance supplement (\#CM4000). Next day, hepatocytes were treated with $\mathrm{ZnCl}_{2}$ or $\mathrm{MgCl}_{2}$ at the concentrations of 100,200 and $400 \mathrm{uM}$ in incubation medium for four hours. Magnesium was used as a negative control, given that zinc and magnesium have opposite roles in the activation of protein tyrosine phosphatase $1 \mathrm{~B}^{10}$. In addition, Okadaic acid (OA) and Metformin (Met) were used as positive controls. OA is an inhibitor of the serine/threonine protein phosphatases (PP2A and PP1), resulting in an elevation of p.AMPKa Thr172 and p.AKT Ser473 levels in hepatocytes ${ }^{11,12}{ }^{2}$. Metformin is an antidiabetic drug that induces phosphorylation of AMPK in liver ${ }^{13}$. Protein lysates were collected using RIPA buffer with Halt Protease \& Phosphatase Inhibitor and subjected for immunoblotting. Cell Viability assay was performed using CellTiter $96 \circledast$ AQueous One Solution Cell Proliferation Assay (MTS) assay (Promega, \#G3580) per manufacturer's protocol.

\section{Generation of SIc39a5 plasmids}

Mouse SIc39a5 ORF sequence was cloned into pIRES2 DsRed-Express2 vector (Clontech, \#PT4079-5). Constructs of SLC39A5 variants were generated using site- 
directed mutagenesis method, with oligos for Y47X (F 5'CCCATTCTCGCCCTACAGGCCAAACAGCTG-3', R 5-CAGCTGTTTGGCCTGTAGG GCGAGAATGGG-3'), R311X (F 5'-GGCCTGAGCCCTCAGTGCCGCAAAAGC-3', R 5'GCTTTTGCGGCACTGAGGGCTCAGGCC-3'), R322X (F 5'-GTTTCGAGATTCCTTCAT TTTCGCCTGCAGCATCT-3', R 5'-AGATGCTGCAGGCGAAAATGAAGGAATCTCGAA AC-3') and M304T (F 5'-AAAGCCCCAGCGTGTTCTCCAGCACAAAGAGCA-3', R 5'TGCTCTTTGTGCTGGAGAACACGCTGGGGCTTT-3'). Mutagenesis was confirmed by Sanger sequencing.

\section{Membrane localization of SLC39A5 using flow cytometry:}

HEK293 cells were plated on a $10 \mathrm{~cm}$ dish at a density of 25,000 cells $/ \mathrm{cm}^{2}$ and incubated at $37^{\circ} \mathrm{C}$ at $5 \% \mathrm{CO}_{2}$ overnight in high glucose DMEM with $10 \% \mathrm{FBS}$ and $1 \%$ Penn Strep (Gibco, \#11965092). Cells were transfected the next day with 10ug plasmid DNA using Xtremegene HP (Roche, \#06366244001) in Opti-MEM (Gibco, \#31985-070). Transfection complexes were incubated at room temperature for 20 minutes and added dropwise to the cells. Cells were incubated at $37^{\circ} \mathrm{C}$. A single media change was performed after 48 hours using Gibco DMEM high glucose and no other supplements. Following 24 hours of incubation at $37^{\circ} \mathrm{C}$ cells were washed with DPBS and dissociated with Cell dissociation Buffer (Gibco, \#13151-014). Cells were resuspended in 0.5\% BSA (Sigma, \#A7030) in DPBS and centrifuged at $200 \mathrm{~g}$ for 5 minutes. BD Biosciecnes CytoFix Fixation buffer (BD, \#554655) was added to each sample and incubated at 4C for 20 minutes. Cells were washed and stained with either anti-human SLC39A5 (Sigma, \#SAB1408465) or isotype control followed by secondary Alexa 488 (ThermoFisher, \#A28175). Cells were washed and re-suspended in 0.5\% BSA in DPBS. All samples were analyzed on a BD FACS Cantoll.

\section{MRE-Iuc assay}

HEK293 cells were plated in 96-well plate at a density of 22,000 cells/well. Cells were transfected with MRE-luc (Promega pGL4.40), hRluc (Promega pGL4.75) and Slc39a5 constructs with XtremeGene HP (Roche) transfection reagent. MRE-binding transcription factor 1 (MTF1) is responsible for expression of metallothioneins (MTs) in 
response to zinc. After 24 hours, medium was changed with $0.5 \%$ charcoal stripped FBS in DMEM + Glutamax (Life Technologies, \#10569010). Twenty-four hours later, the cultures were exposed to $\mathrm{Zn}^{2+}$ for 6 hours. Luciferase activity was measured using DualGlo luciferase assay system (Promega, \#E2920). Results were expressed as the relation of firefly luciferase activity to Renilla luciferase activity.

\section{Live staining \& Immunofluorescence}

HEK293 cells were plated on Ibidi chamber slides (Ibidi, \#80427) coated with $100 \mu \mathrm{g} / \mathrm{mL}$ of poly-L-lysine. Live cells staining was performed by diluting primary antibody (Polyclonal anti-SLC39A5, Sigma SAB1408465, 1:100) in cold culture medium and incubated with the cells for 2 hours at $4^{\circ} \mathrm{C}$. Slides were washed with cold PBS $3 \times 10$ minutes at $4{ }^{\circ} \mathrm{C}$ with gentle shaking. Cells were fixed with $2 \%$ paraformaldehyde at room temperature for 5 minutes and washed with PBS. Wheat germ agglutinin in HBSS was added to cells for 10 minutes at room temperature and then blocked with $5 \%$ goat serum. Secondary antibody was applied for 45 minutes at room temperature. Cells were washed and mounted with ProLong diamond antifade solution and imaged using Zeiss AxioObserver LSM880.

\section{Hepatic $\beta$-hydroxybutyrate assay}

$\beta$-OHB (Sigma, \#MAK041) was measured in mouse liver tissue by colorimetric assays per manufacturer's guidelines. Briefly, liver samples $(10 \mathrm{mg} / \mathrm{sample})$ were homogenized in 4 volumes of cold $\beta-O H B$ assay buffer. Samples were centrifuged at $13,000 \mathrm{~g}$ for 10 minutes at $4^{\circ} \mathrm{C}$ and supernatant was used for analyses. Concentration is determined by a coupled enzyme reaction which results in a colorimetric $(450 \mathrm{~nm})$ product proportional to the $\beta$-OHB present.

\section{Protein Phosphatase Assay}

Cultured hepatocyte lysates and tissue homogenates were prepared by Lysis Reagent (Invitrogen, \#M10510) with protease inhibitor cocktail (Sigma, \#P8340). Protein lysates were centrifuged at $4^{\circ} \mathrm{C}$ for 15 minutes and protein concentration was determined using Pierce TM BCA protein assay kit. Phosphatase activity was measured using 
RediPlate $^{\mathrm{TM}} 96$ EnzChek $^{\mathrm{TM}}$ Tyrosine Phosphatase and Serine/Threonine Phosphatase Assay Kit (Invitrogen, \#R22067, R33700) per manufacturer's instructions. Samples were incubated in RediPlate for 60 mins before reading by fluorescence microplate with excitation/emission at 358/455nm (Spectramax M4, Molecular Devices).

\section{Superoxide Dismutase (SOD) assay}

SOD activity was measured in mouse liver tissues using SOD activity kit (ENZO, \#ADI-900-157). Briefly, liver samples (30 mg/sample) were homogenized in 10 volumes of cell extraction buffer. Samples were centrifuged at $10,000 \mathrm{~g}$ for 10 minutes at $4^{\circ} \mathrm{C}$ and supernatant was used for analysis. Protein concentration was determined by Pierce TM BCA protein assay kit (Thermo Scientific, \#23225). Samples were diluted to 50ug/25ul for assay, according to protocol.

\section{Statistical analysis}

Results are shown as box plots with individual values. Statistical analysis was performed using GraphPad Prism 8 software. Analysis for Slc39a5-/-; Lepr/- mice and corresponding control mice was performed using one-way ANOVA, followed by post hoc Tukey's tests. Analysis for HFFD and NASH mouse studies was performed using twoway ANOVA, followed by post hoc Tukey's tests. Statistical significance reported when $p<0.05$. Sample sizes, statistical test and significance are described in each figure legend.

\section{References}

[1] Eastwood SV, Mathur R, Atkinson M, Brophy S, Sudlow C, Flaig R, et al. Algorithms for the Capture and Adjudication of Prevalent and Incident Diabetes in UK Biobank. PLoS One 2016;11:e0162388.

[2] Lotta LA, Wittemans LBL, Zuber V, Stewart ID, Sharp SJ, Luan J, et al. Association of Genetic Variants Related to Gluteofemoral vs Abdominal Fat Distribution With Type 2 Diabetes, Coronary Disease, and Cardiovascular Risk Factors. JAMA 2018;320:2553-2563.

[3] Berglund G, Elmstahl S, Janzon L, Larsson SA. The Malmo Diet and Cancer Study. Design and feasibility. J Intern Med 1993;233:45-51.

[4] Mbatchou J, Barnard L, Backman J, Marcketta A, Kosmicki JA, Ziyatdinov A, et al. Computationally efficient whole-genome regression for quantitative and binary traits. Nat Genet 2021. 
medRxiv preprint doi: https://doi.org/10.1101/2021.12.08.21267440; this version posted December 9, 2021. The copyright holder for this preprint (which was not certified by peer review) is the author/funder, who has granted medRxiv a license to display the preprint in perpetuity.

All rights reserved. No reuse allowed without permission.

[5] Poueymirou WT, Auerbach W, Frendewey D, Hickey JF, Escaravage JM, Esau L, et al. F0 generation mice fully derived from gene-targeted embryonic stem cells allowing immediate phenotypic analyses. Nat Biotechnol 2007;25:91-99.

[6] Valenzuela DM, Murphy AJ, Frendewey D, Gale NW, Economides AN, Auerbach W, et al. High-throughput engineering of the mouse genome coupled with high-resolution expression analysis. Nat Biotechnol 2003;21:652-659.

[7] Liang W, Menke AL, Driessen A, Koek GH, Lindeman JH, Stoop R, et al. Establishment of a general NAFLD scoring system for rodent models and comparison to human liver pathology. PLoS One 2014;9:e115922.

[8] Folch J, Lees M, Sloane Stanley GH. A simple method for the isolation and purification of total lipides from animal tissues. J Biol Chem 1957;226:497-509.

[9] Carr TP, Andresen CJ, Rudel LL. Enzymatic determination of triglyceride, free cholesterol, and total cholesterol in tissue lipid extracts. Clin Biochem 1993;26:39-42.

[10] Bellomo E, Abro A, Hogstrand C, Maret W, Domene C. Role of Zinc and Magnesium lons in the Modulation of Phosphoryl Transfer in Protein Tyrosine Phosphatase 1B. J Am Chem Soc 2018;140:4446-4454.

[11] Samari HR, Moller MT, Holden L, Asmyhr T, Seglen PO. Stimulation of hepatocytic AMPactivated protein kinase by okadaic acid and other autophagy-suppressive toxins. Biochem J 2005;386:237-244.

[12] Galbo T, Olsen GS, Quistorff B, Nishimura E. Free fatty acid-induced PP2A hyperactivity selectively impairs hepatic insulin action on glucose metabolism. PLoS One 2011;6:e27424. [13] Howell JJ, Hellberg K, Turner M, Talbott G, Kolar MJ, Ross DS, et al. Metformin Inhibits Hepatic mTORC1 Signaling via Dose-Dependent Mechanisms Involving AMPK and the TSC Complex. Cell Metab 2017;25:463-471. 
A
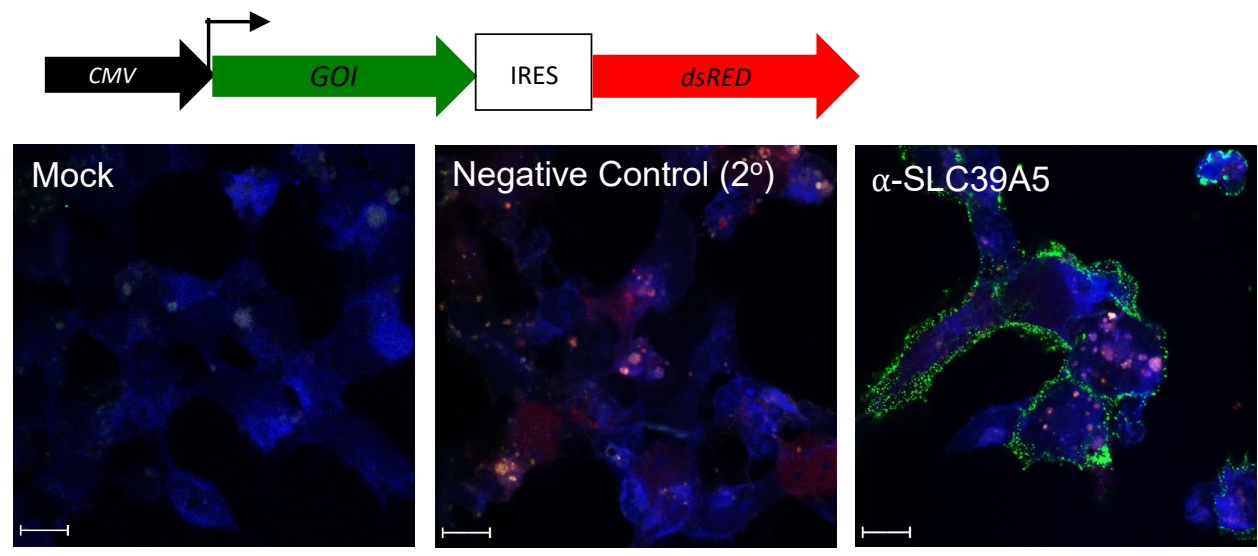

B

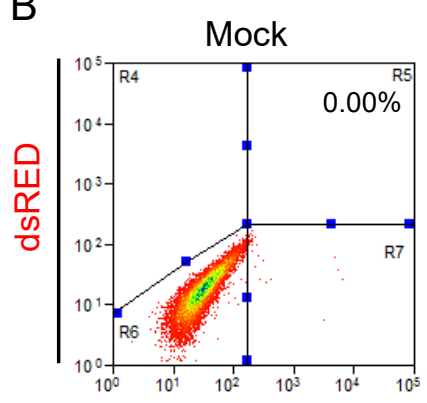

Negative Control $\left(2^{\circ}\right)$

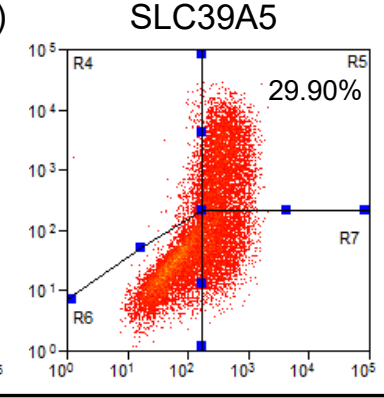

C

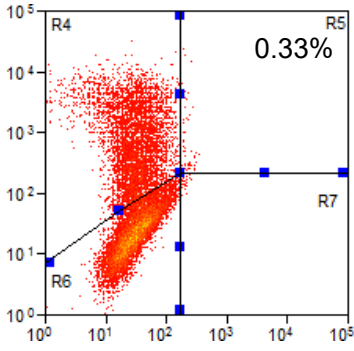

Alexa 488 (SLC39A5)

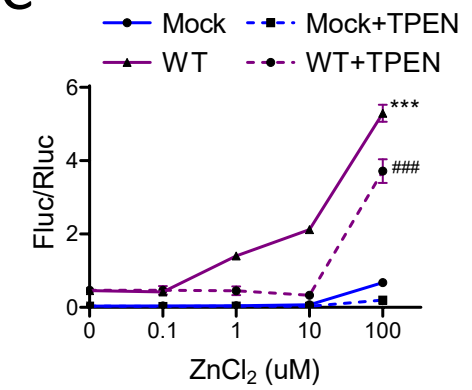

D
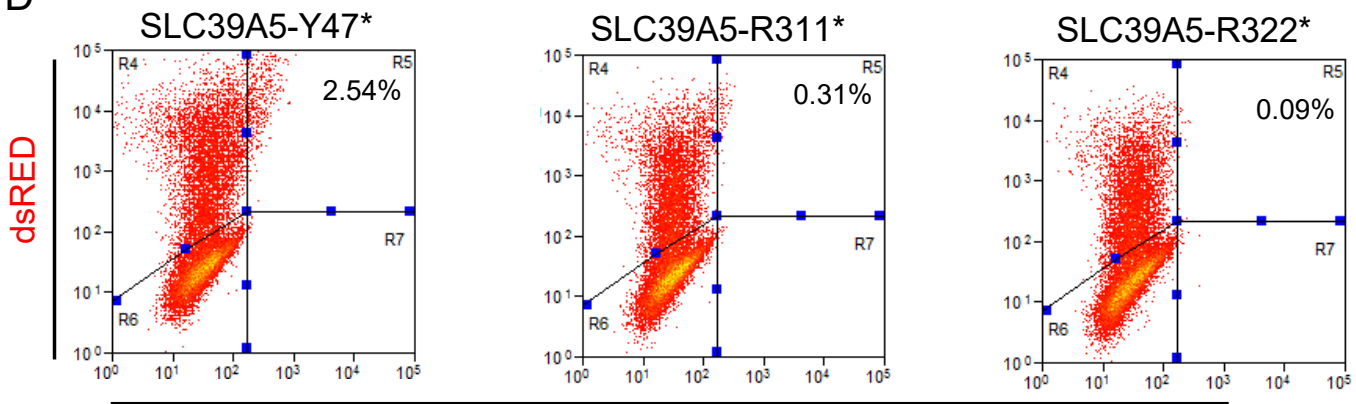

Alexa 488 (SLC39A5)

E
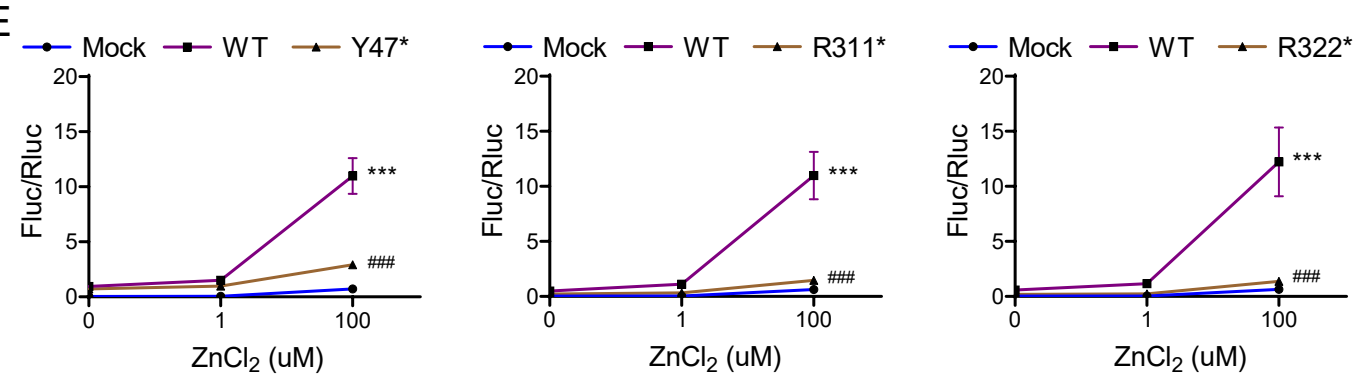

Suppl. Fig. 1. SLC39A5 pLOF variants p.Y47*(c.141C>G), p.R311*(c.931C>T), and p.R322*(c.964C>T) encode for non-functional proteins. HEK293 cell transfected with expression constructs encoding SLC39A5 wild-type (WT), Y47*, R311* and R322* variants. (A, B) Immunostaining and FACS analysis demonstrating WT SLC39A5 localization to the cell surface. (C) Overexpression of WT SLC39A5 results in $\mathrm{Zn}^{2+}$ mediated MRE activation in a dose dependent manner, $n=4$. (D) FACS analyses demonstrating that cell surface expression of

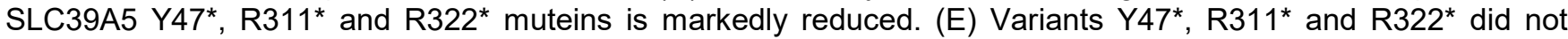
mediate $\mathrm{Zn}^{2+}$ induction of MRE, $\mathrm{n}=8$, Statistical comparison to Mock and WT, respectively: ${ }^{* *} \mathrm{P}<0.001$, \#\#\# $<$ 0.001, two-way ANOVA with post hoc Tukey's test. Metal regulatory element (MRE), firefly luciferase (Fluc), renilla luciferase (Rluc), cytomegalovirus (CMV), gene of interest (GOI), internal ribosome entry site (IRES). 
A

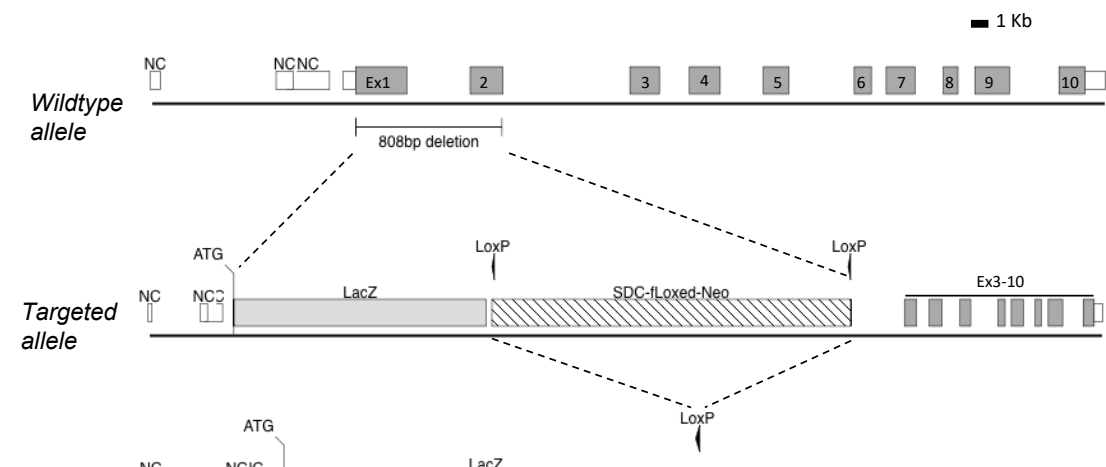

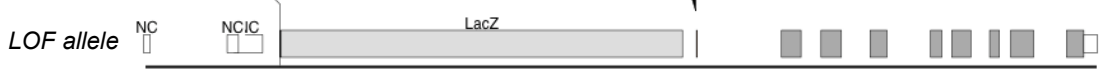

$\square$ untranslated region

coding region
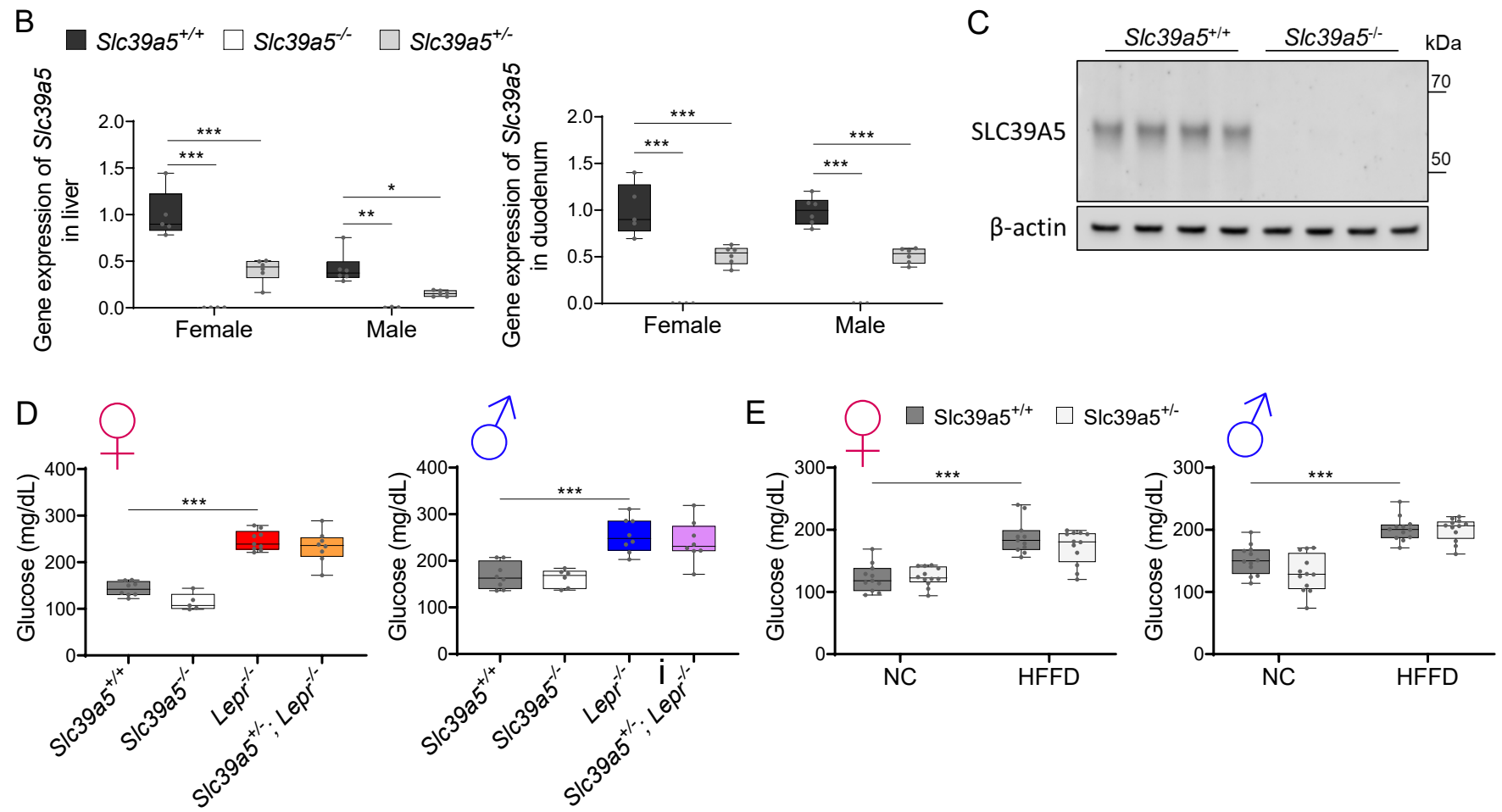

Suppl. Fig. 2. Generation and characterization of the S/c39a5/ mice. (A) Schematic representation of the S/c39a5 null allele. (B) S/c39a5 gene expression in liver and duodenum of S/c39a5 $5^{-1-}$ mice at 20 weeks of age, $n=3-6$. (C) Immunoblotting analyses demonstrating absence of SLC39A5 protein in liver of S/c39a5-- mice at 34 weeks of age. (D-E) Heterozygous loss of S/c39a5 does not reduce fasting blood glucose in (D) Lepr/- mice (at 20 weeks of age) and in (E) mice challenged with high fat high fructose diet (HFFD) for 18 weeks. ${ }^{*} P<0.05,{ }^{* *} P<0.01,{ }^{* *} P<$ 0.001 , two-way ANOVA with post hoc Tukey's test. 
A

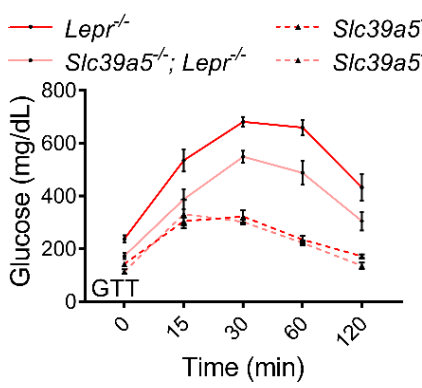

B

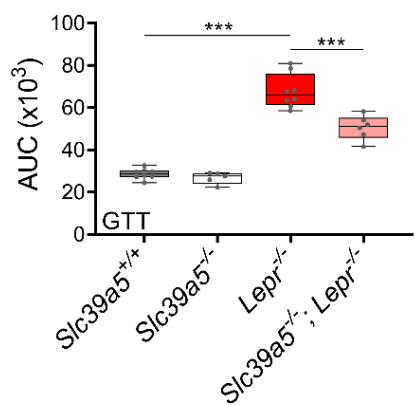

F

E
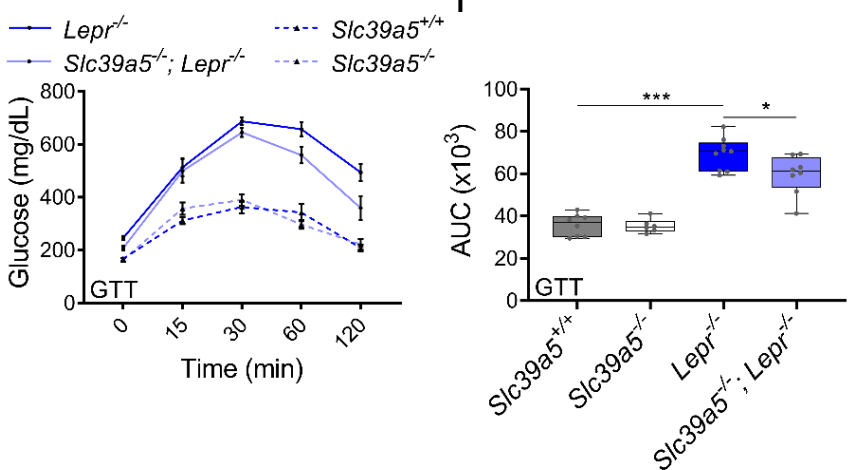

C

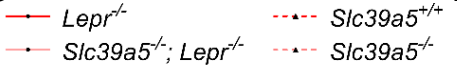

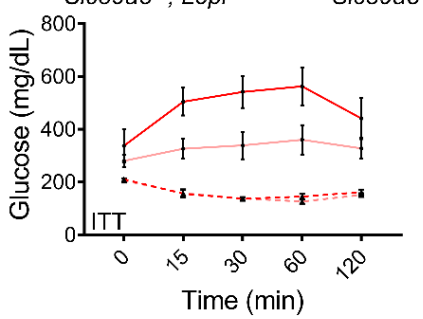

G

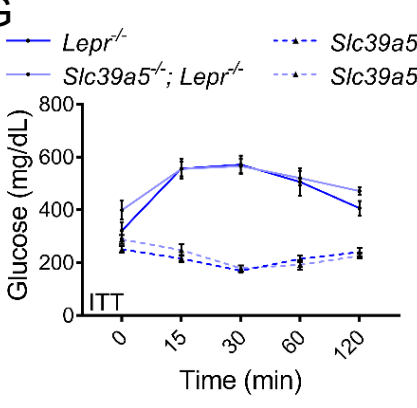

K
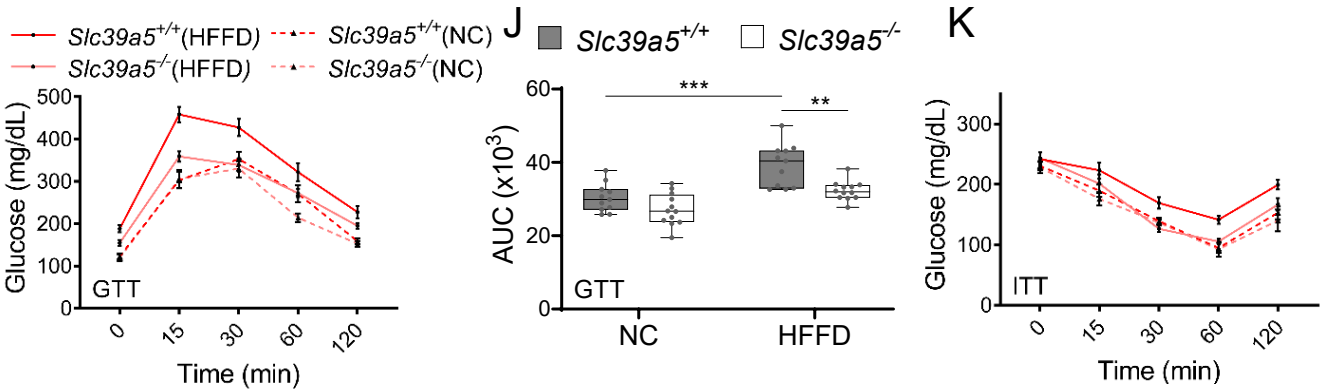

M
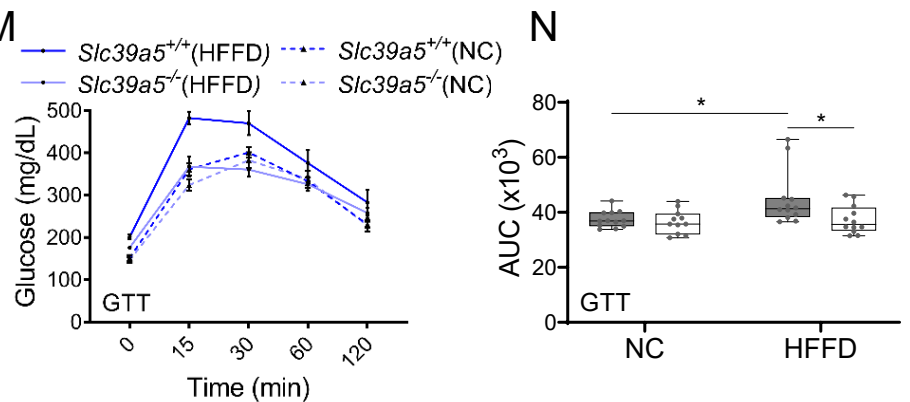

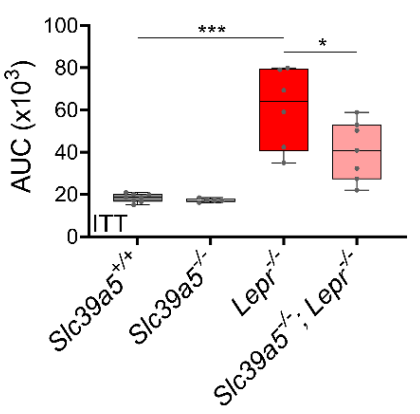

$\mathrm{H}$
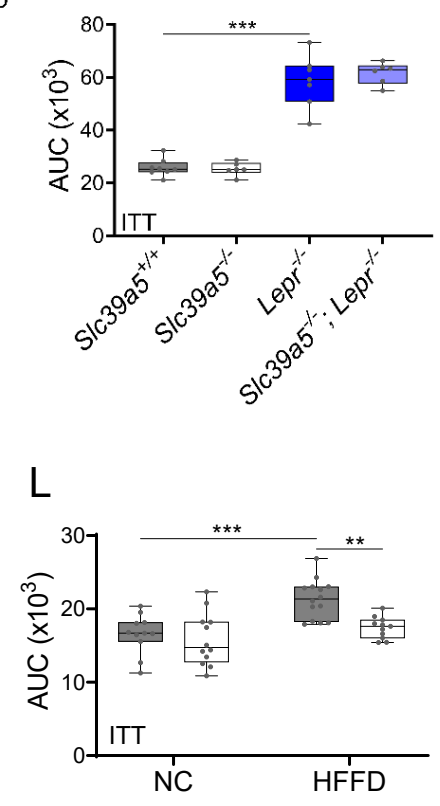

D

L

P

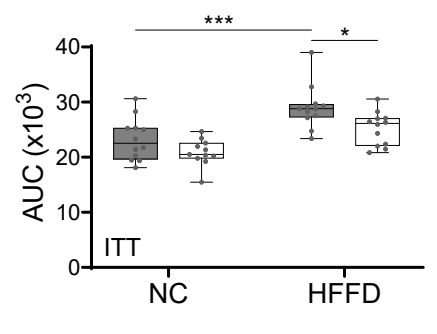

Suppl. Fig. 3. Loss of S/c39a5 improves glycemic traits in Lepr/- mice and in mice challenged with high

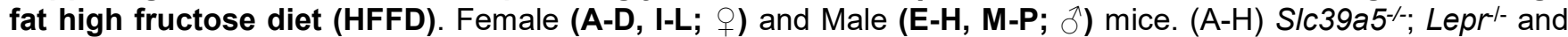
corresponding control mice. (A-B, E-F) Oral glucose tolerance test (GTT) after 16 hour fasting, at 20 weeks. (C-D, G-H) Intraperitoneal insulin tolerance test (ITT), at 33 weeks. $n=5-8$. ${ }^{*} \mathrm{P}<0.05,{ }^{* *} \mathrm{P}<0.01,{ }^{* * *} \mathrm{P}<0.001$, one-way ANOVA with post hoc Tukey's test. (I-P) HFFD mice. (I-J, M-N) GTT after 16 hours fasting, at 18 weeks. (K-L. OP) ITT after 4 hour fasting, at 27 weeks. $n=11-12$. ${ }^{*} P<0.05,{ }^{* *} P<0.01,{ }^{* * *} P<0.001$, two-way ANOVA with post hoc Tukey's test. Area under curve (AUC). Numeric data is summarized in Suppl. Table 4 and 5. 
A

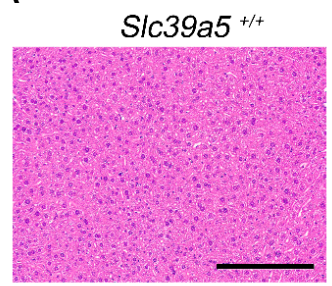

C

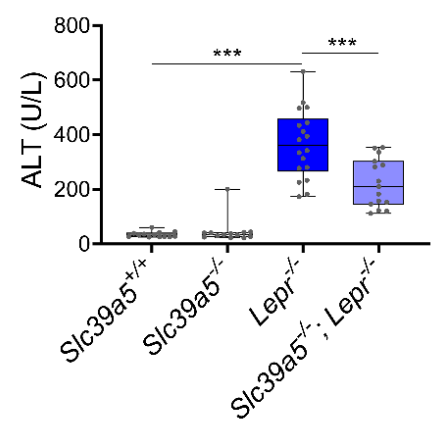

D

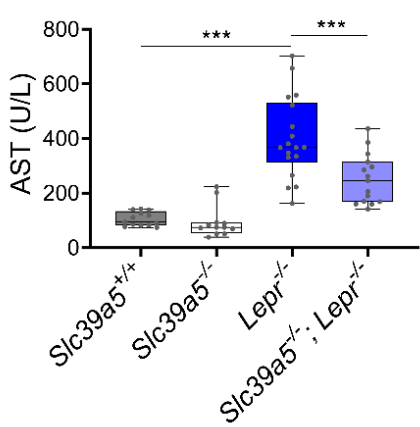

E

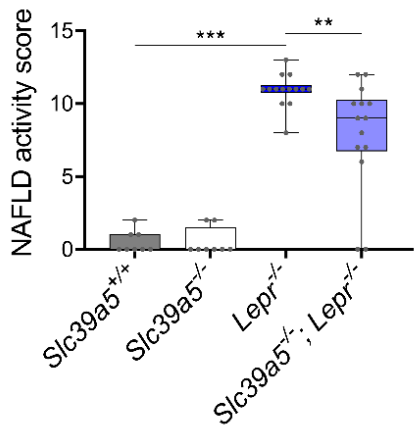

F
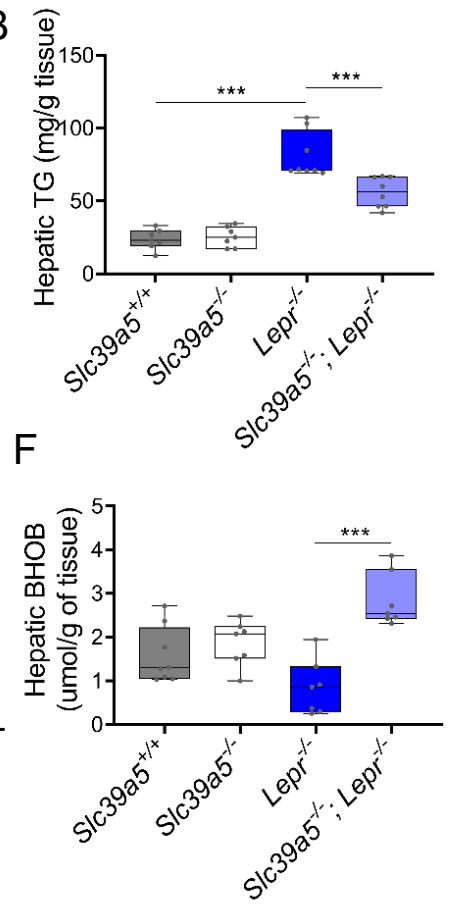

$\mathrm{H}$

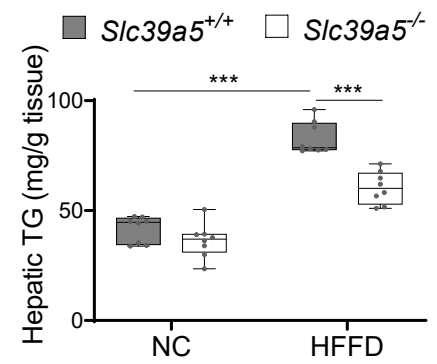

G

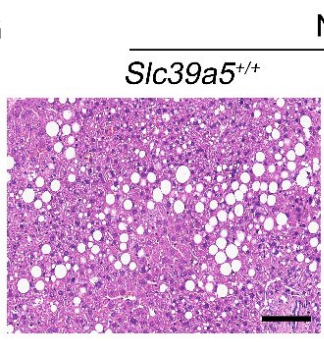

NC Slc39a5

Slc39a5 $5^{+/+}$

HFFD

Slc39a5
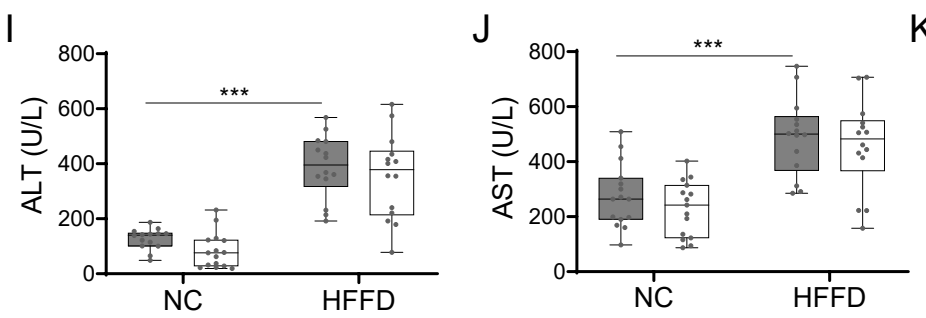

K

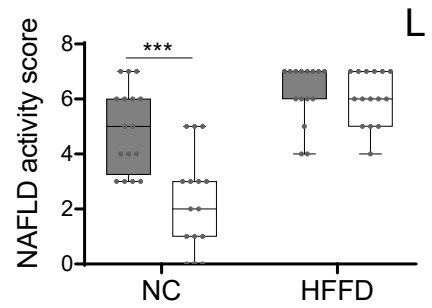

L

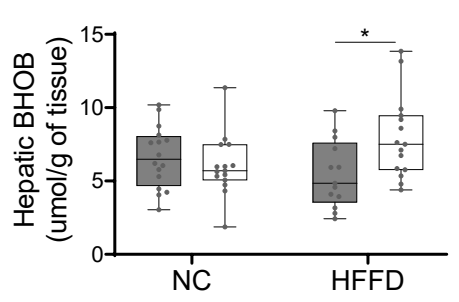

Suppl. Fig. 4. Loss of SIc39a5 improves liver function and steatosis in Lepr/- male mice and reduces hepatic triglyceride in male mice challenged with high fat high fructose diet (HFFD). S/c39a5-; Lepr/- and corresponding control mice (A-F) were sacrificed after 16 hours fasting at 34 weeks of age. (G-L) S/c $39 a 5^{-/}$and corresponding control mice were fed HFFD or NC for 30 weeks and sacrificed after 16 hours fasting. (A, G)

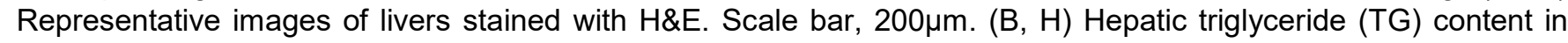
explanted liver samples at endpoint. (C, I) Serum ALT. (D, J) Serum AST. (E, K) NAFLD activity score, (F, L) Hepatic beta-hydroxybutyrate (BHOB). ${ }^{*} \mathrm{P}<0.05,{ }^{* *} \mathrm{P}<0.01,{ }^{* * *} \mathrm{P}<0.001$, Slc39a5 ${ }^{--}$; Lepr $r^{-}$mice: one-way ANOVA with post hoc Tukey's test, HFFD: two-way ANOVA with post hoc Tukey's test. Numeric data is summarized in Suppl. Table 4 and 5. 
A

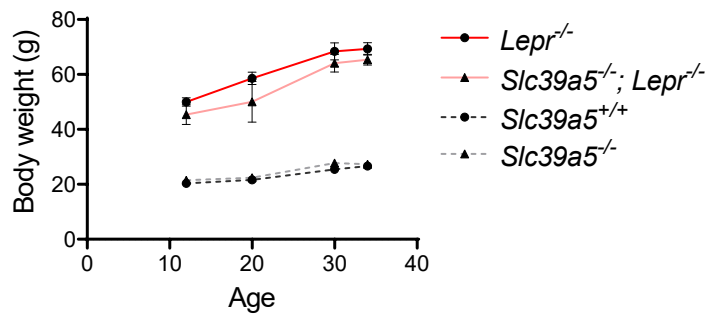

C

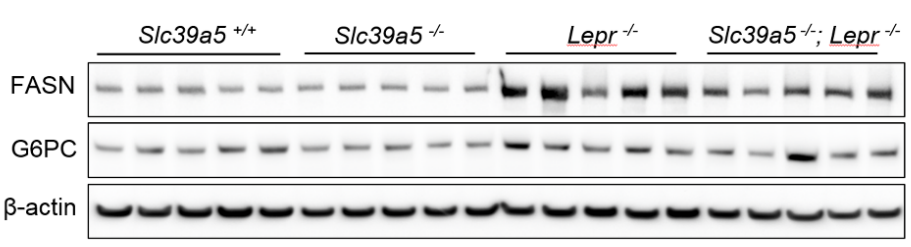

E

하요

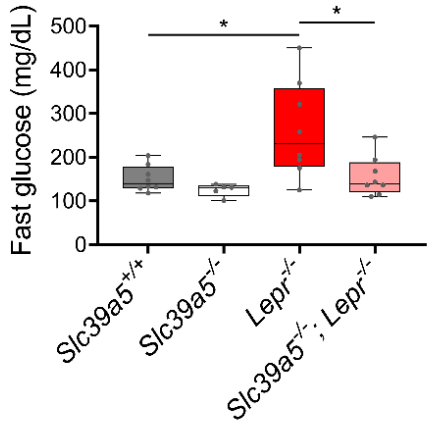

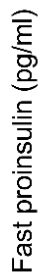
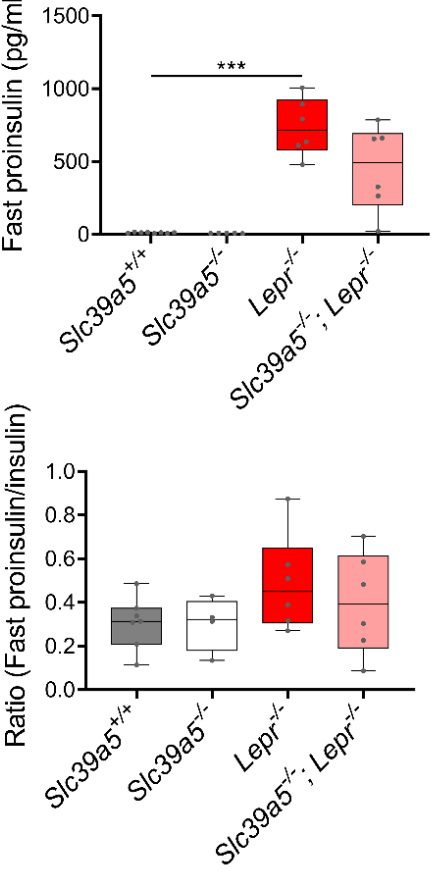

B

D

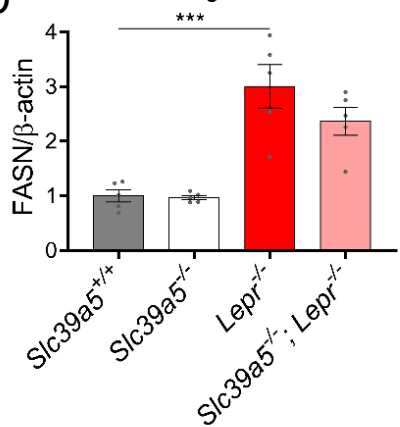

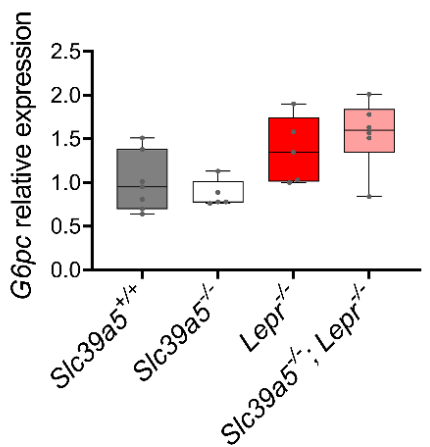

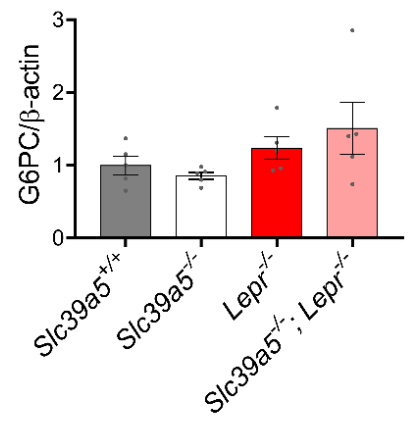

Suppl. Fig. 5. Loss of SIc39a5 reduces hepatic fatty acid synthase expression but does not change the insulin profile of female Lepr/- mice. (A) Longitudinal body weight. (B-D) Analyses were done on explanted liver samples collected after 16 hour fasting at 34 weeks of age. (B) Hepatic expression of fatty acid synthase (Fasn) and glucose-6phosphatase (G6pc). (C) Hepatic FASN and G6PC protein levels. (D) Densitometric analysis of hepatic FASN and G6PC. (E) Serum insulin profile upon fasting at 34 weeks of age. (F) Serum insulin profile in fed state at 32 weeks of age. ${ }^{*} P<0.05,{ }^{* *} P<0.01,{ }^{* * *} P<0.001$, ANOVA with post hoc Tukey's test. 
A

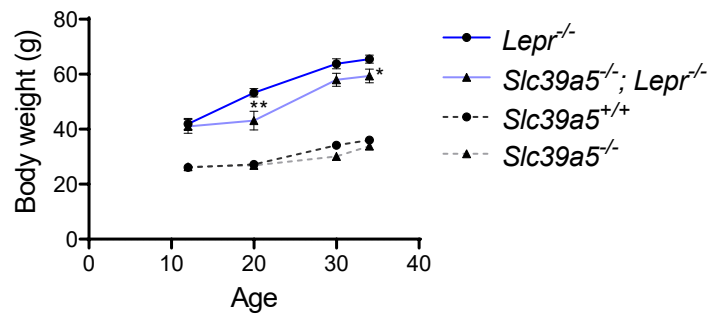

C

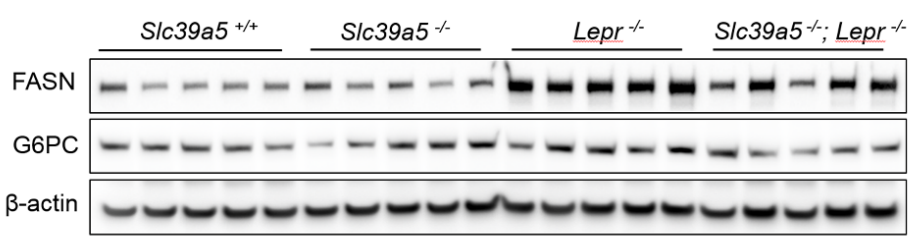

E
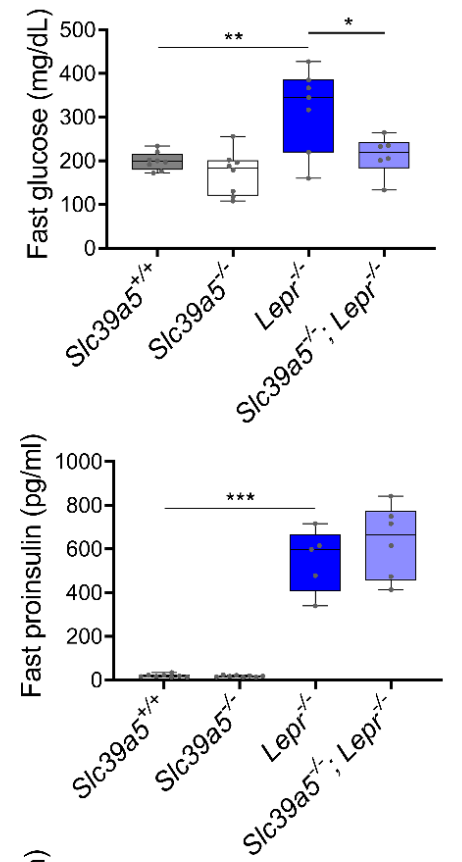

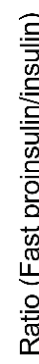

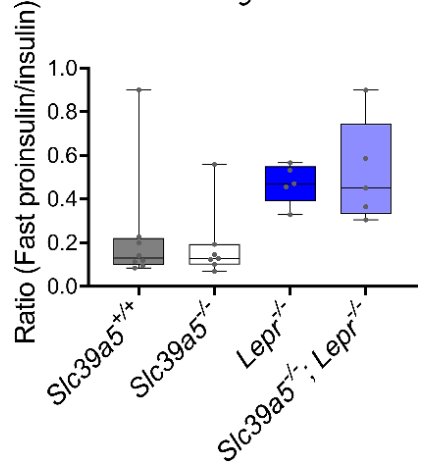

$\mathrm{F}$
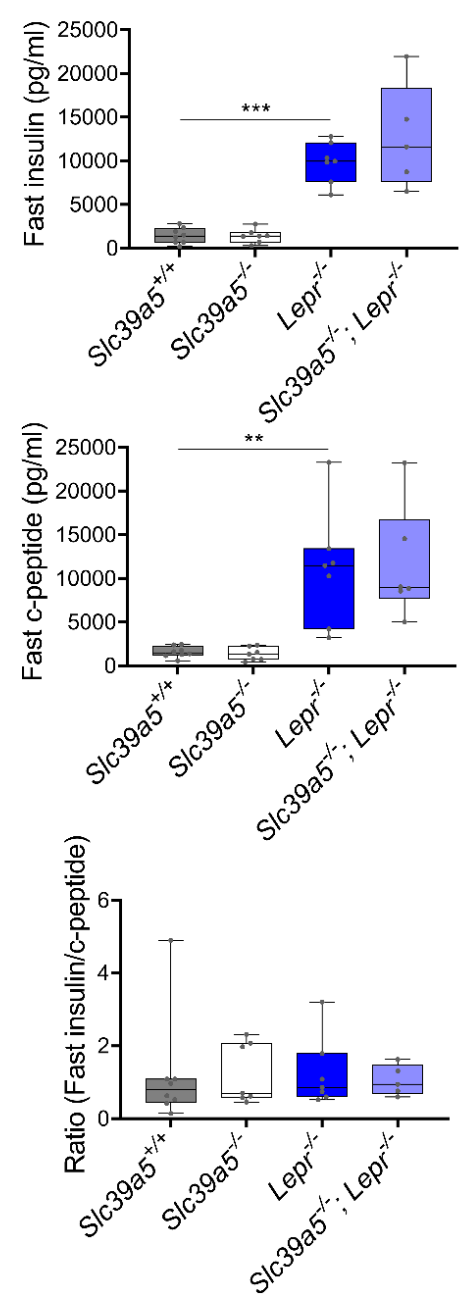

$B$

D
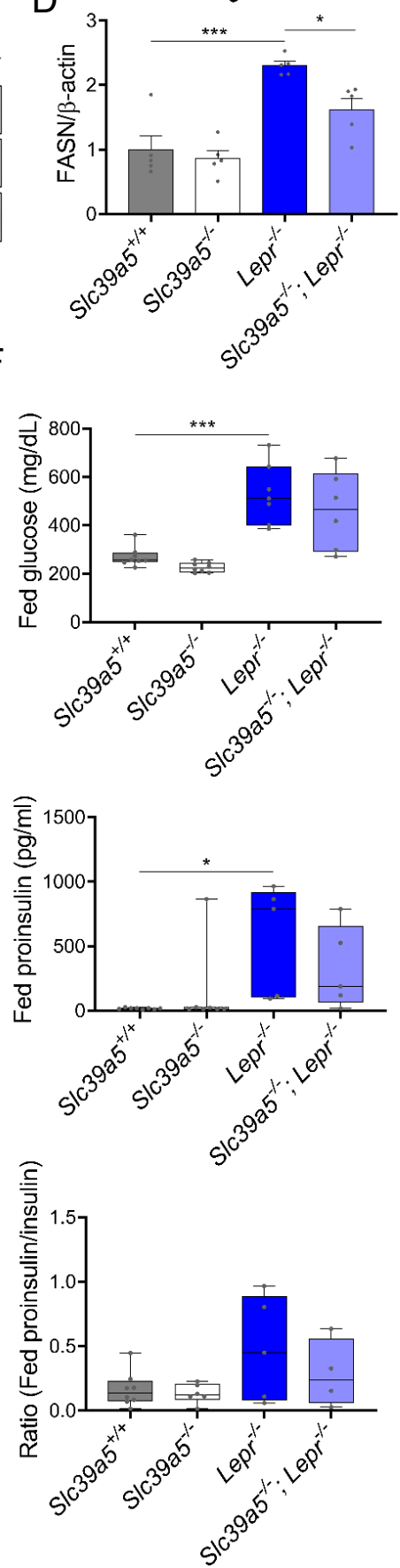
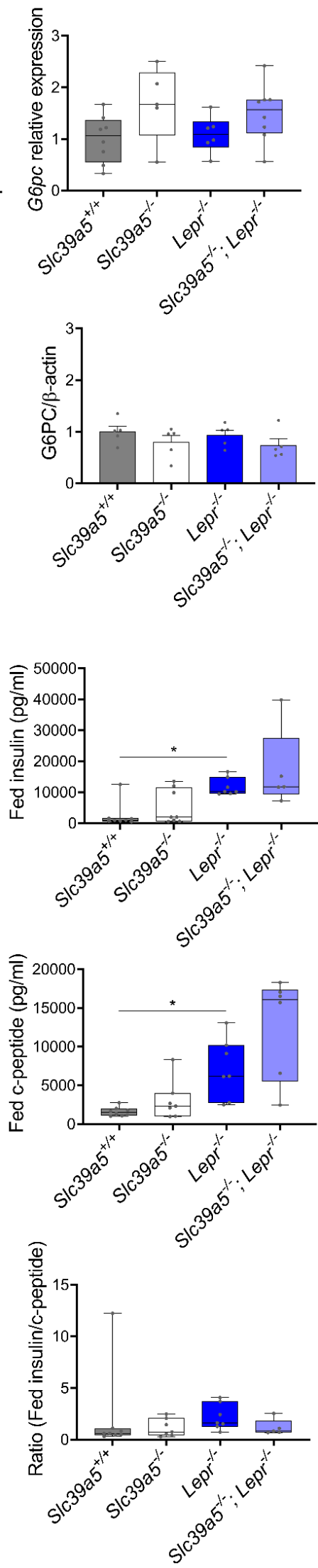

Suppl. Fig. 6. Loss of S/c39a5 reduces hepatic fatty acid synthase expression but does not change insulin profile of male Lepr/- mice. (A) Longitudinal body weight. (B-D) Analyses were done on explanted liver samples collected after 16 hour fasting at 34 weeks of age. (B) Hepatic expression of Fasn and G6pc. (C) Hepatic FASN and G6PC protein levels. (D) Densitometric analysis of hepatic FASN and G6PC. (E) Serum insulin profile upon fasting at 34 weeks of age. (F) Serum insulin profile in fed state at 32 weeks of age. ${ }^{*} P<0.05,{ }^{* *} P<0.01,{ }^{* * *} P<0.001$, ANOVA with post hoc Tukey's test. 


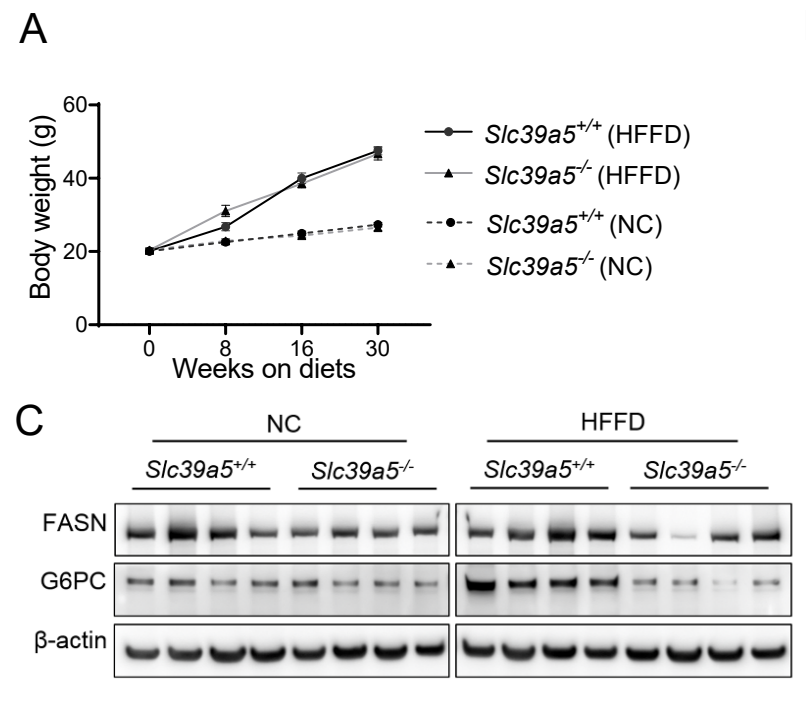

$\mathrm{E}$
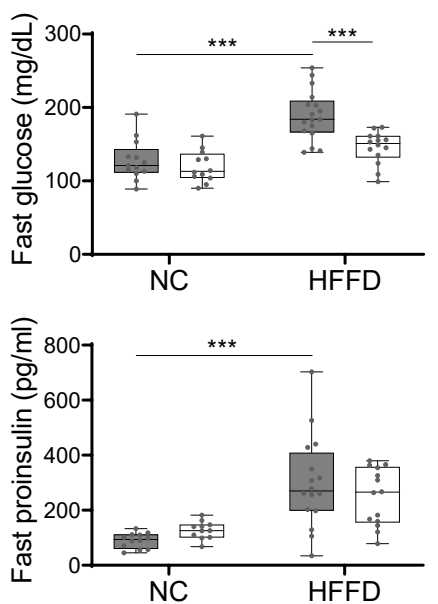

$\stackrel{. \subseteq}{\frac{5}{5}}$

G
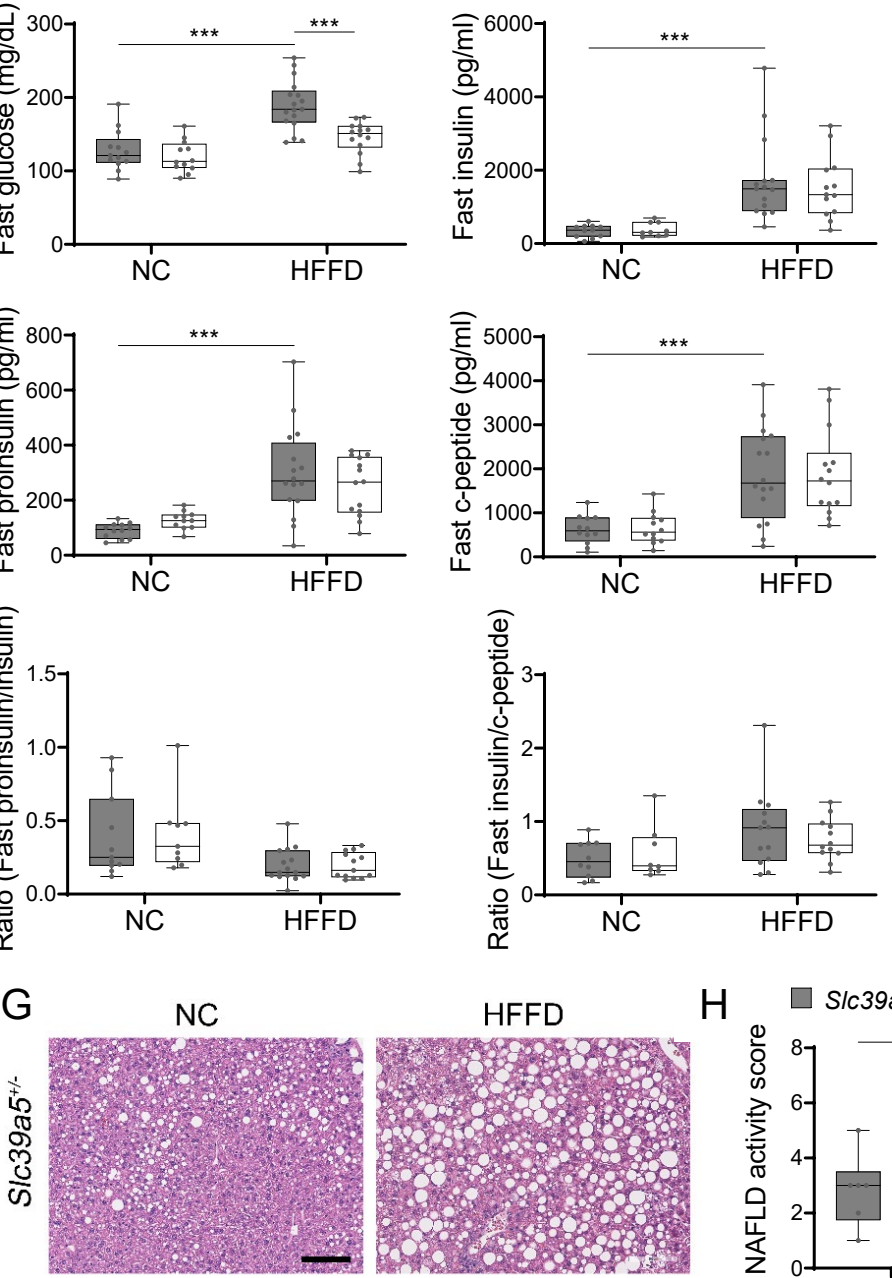

B
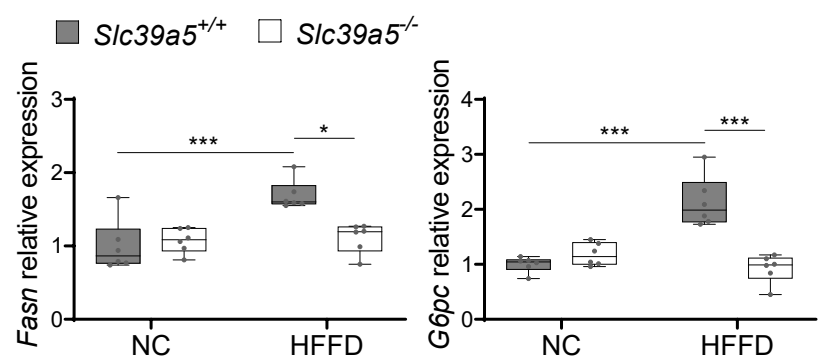

D
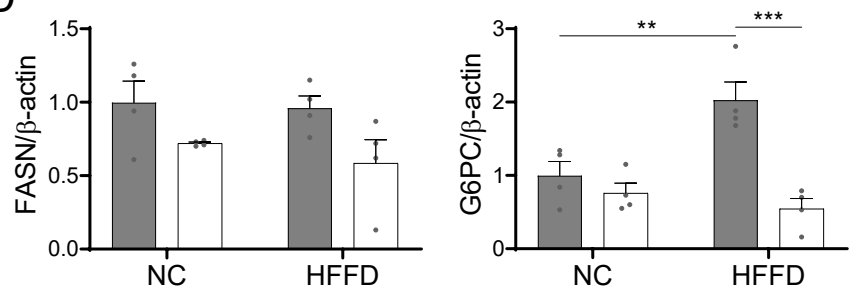

$\mathrm{F}$
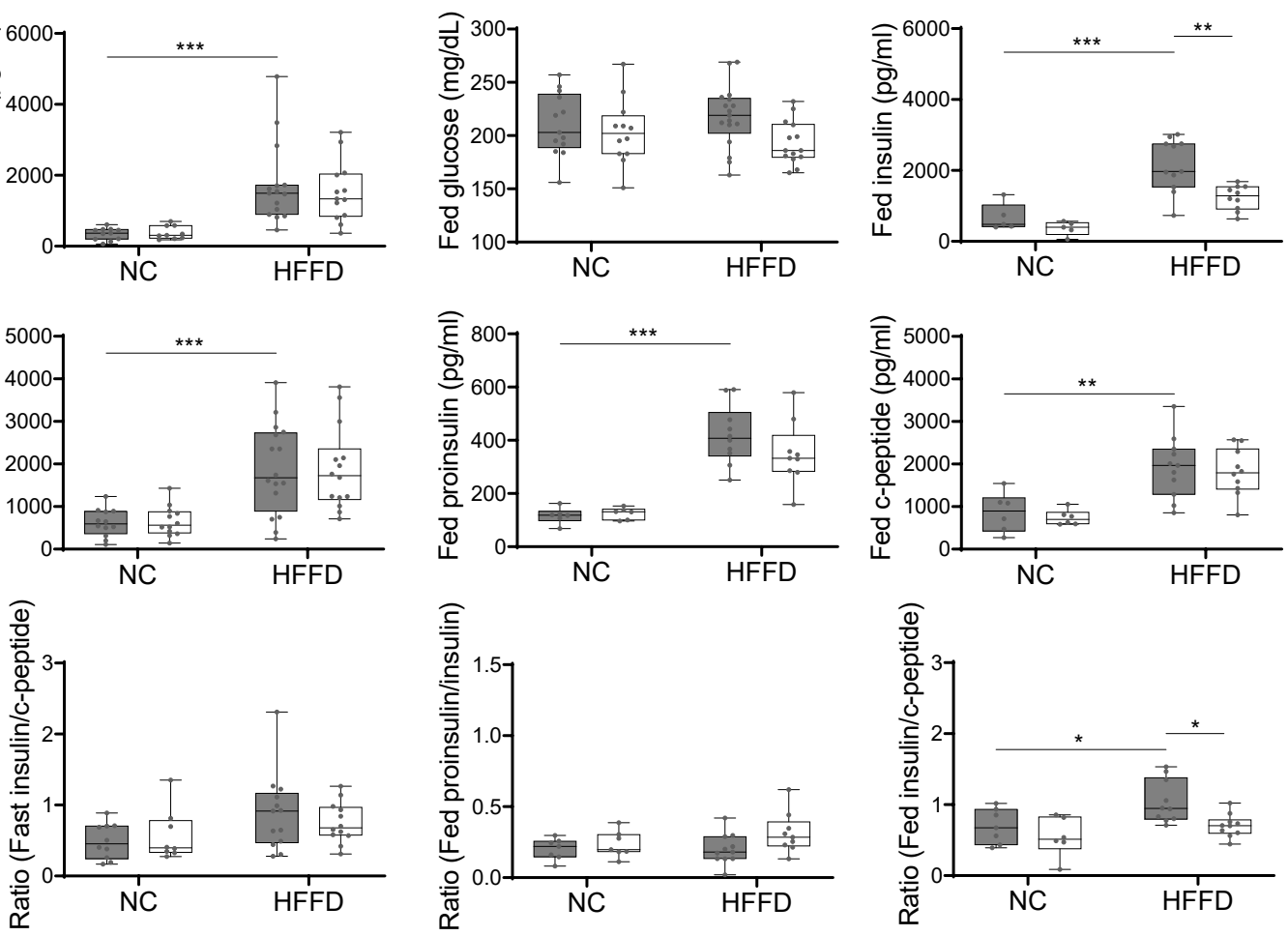

Suppl. Fig. 7. Loss of SIc39a5 reduces hepatic fatty acid synthase expression but does not change insulin profile in female mice challenged with high fat high fructose diet (HFFD). (A) Longitudinal body weight during dietary intervention. (B-D) Analyses were done on explanted liver samples collected after 16 hour fasting in mice fed HFFD or NC for 30 weeks. (B) Hepatic expression of Fasn and G6pc. (C) Hepatic FASN and G6PC protein levels. (D) Densitometric analysis of hepatic FASN and G6PC. (E) Fasting serum insulin profile. (F) Fed serum insulin profile. (G) Representative images of Slc39a5 ${ }^{+/}$livers stained with H\&E. Scale bar, 100um. (H) NAFLD activity score. ${ }^{*} \mathrm{P}<0.05$, ${ }^{* *} \mathrm{P}<0.01,{ }^{* * *} \mathrm{P}<0.001$, two-way ANOVA with post hoc Tukey's test. 
A

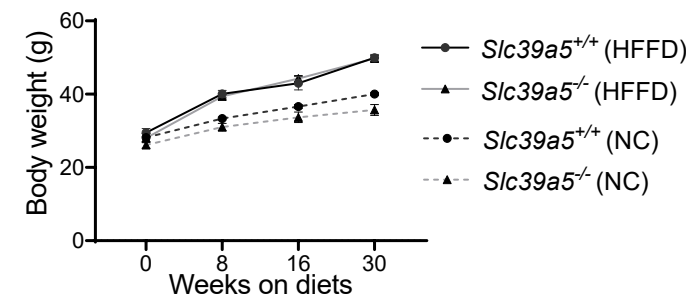

C

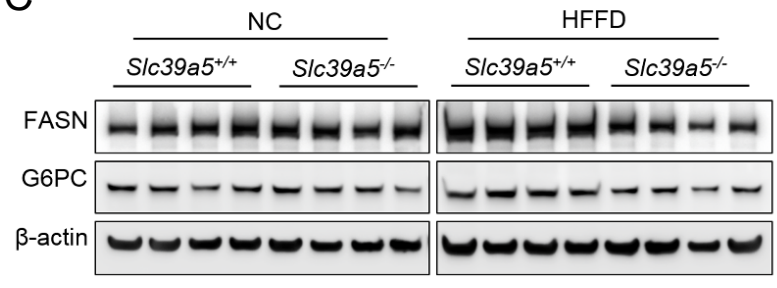

$\mathrm{E}$
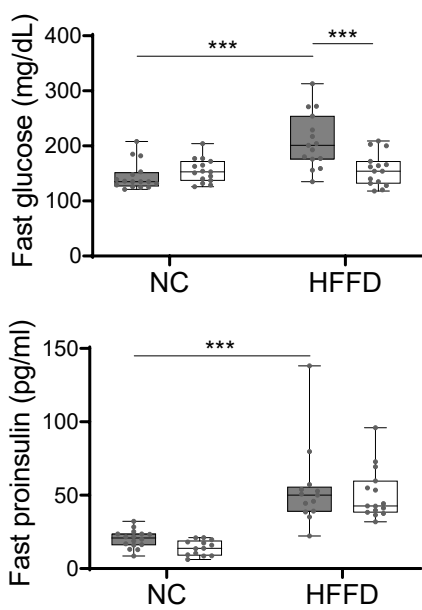

禀

G

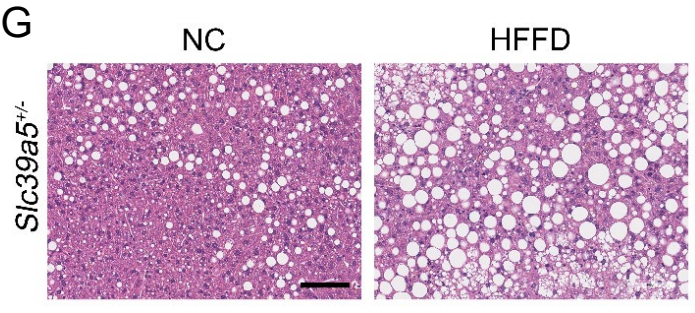

B
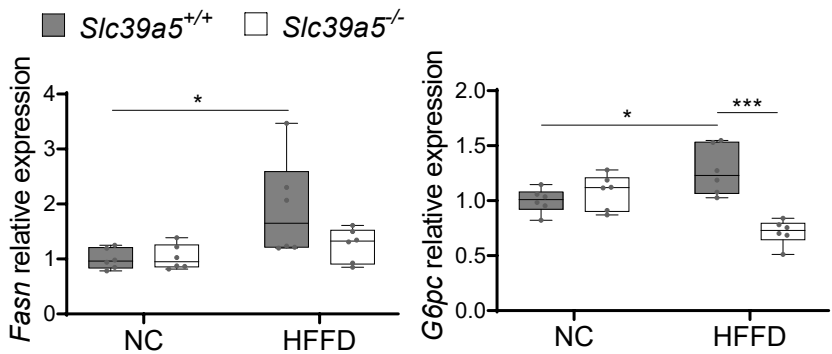

D
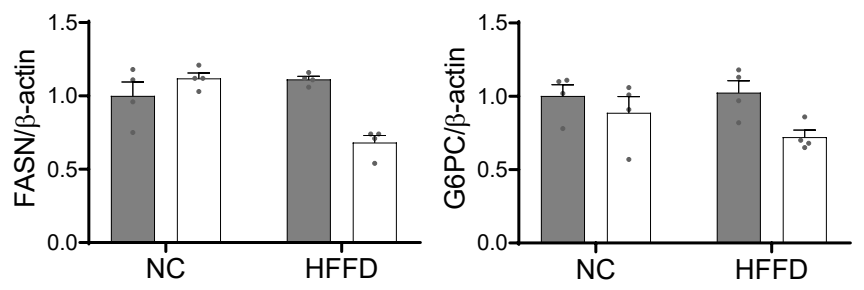

$\mathrm{F}$
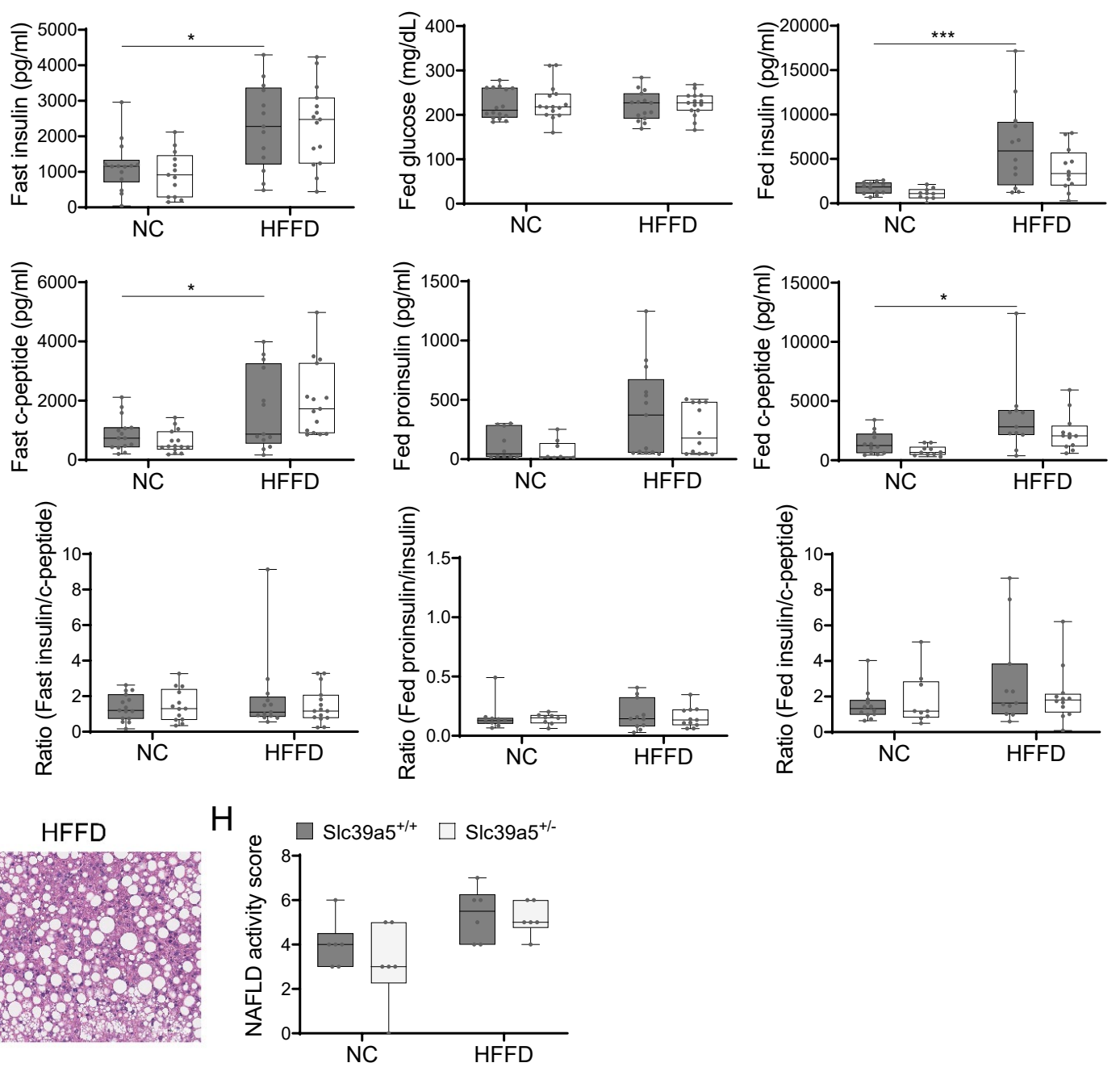

Suppl. Fig. 8. Loss of SIc39a5 reduces hepatic fatty acid synthase expression but does not change insulin profile in male mice challenged with high fat high fructose diet (HFFD). (A) Longitudinal body weight during dietary intervention. (B-D) Analyses were done on explanted liver samples collected after 16 hour fasting in mice fed HFFD or NC for 30 weeks. (B) Hepatic expression of Fasn and G6pc. (C) Hepatic FASN and G6PC protein levels. (D) Densitometric analysis of hepatic FASN and G6PC. (E) Fasting serum insulin profile. (F) Fed serum insulin profile. (G) Representative images of Slc39a5 ${ }^{+/}$livers stained with H\&E. Scale bar, 100um. (H) NAFLD activity score. ${ }^{*} \mathrm{P}<0.05$, ${ }^{* *} \mathrm{P}<0.01,{ }^{* * *} \mathrm{P}<0.001$, two-way ANOVA with post hoc Tukey's test. 
A

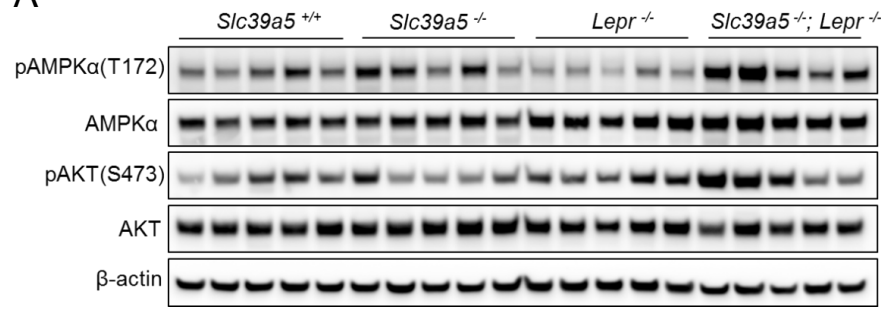

B

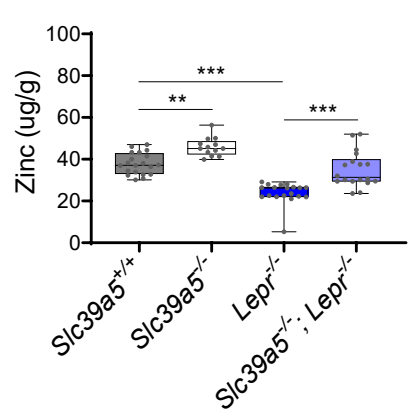

C

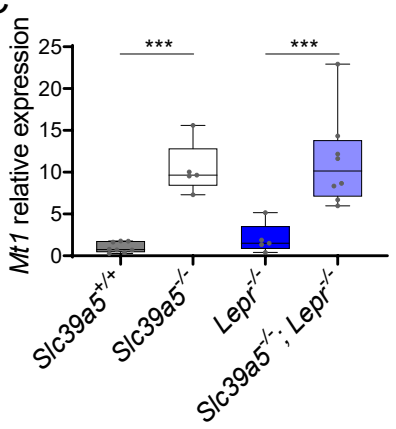

D

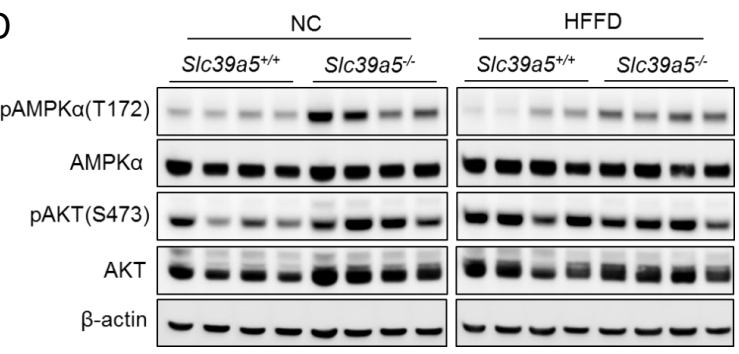

$\mathrm{E}$

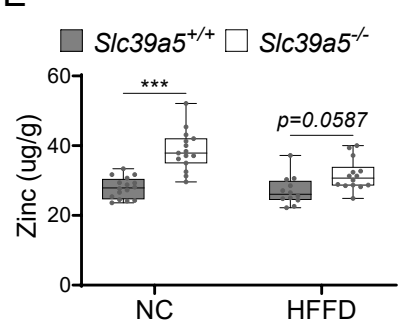

$\mathrm{F}$

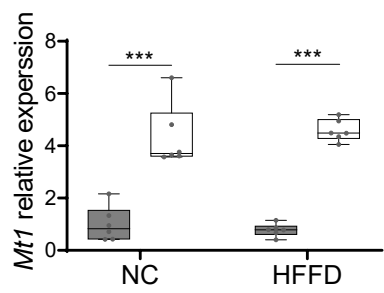

Suppl. Fig. 9. Loss of S/c39a5 results in elevated hepatic zinc and activation of hepatic AMPK signaling in congenital and diet-induced obesity models. Analyses were done on explanted liver samples collected from male mice after 16 hour fasting at endpoint of congenital (A-C) and diet-induced obesity (D-F). (A, D) Immunoblot analysis of hepatic AMPK and AKT activation. (B, E) Hepatic zinc measurements $(n=10-21)$. (C, F) Hepatic Mt1 gene expression. ${ }^{*} \mathrm{P}<0.05,{ }^{* *} \mathrm{P}<0.01,{ }^{* * *} \mathrm{P}<0.001$, ANOVA with post hoc Tukey's test. 
A

C

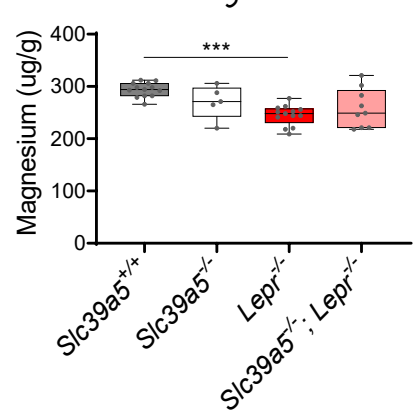

D

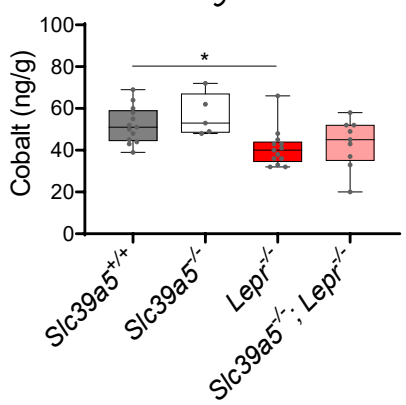

ठ F
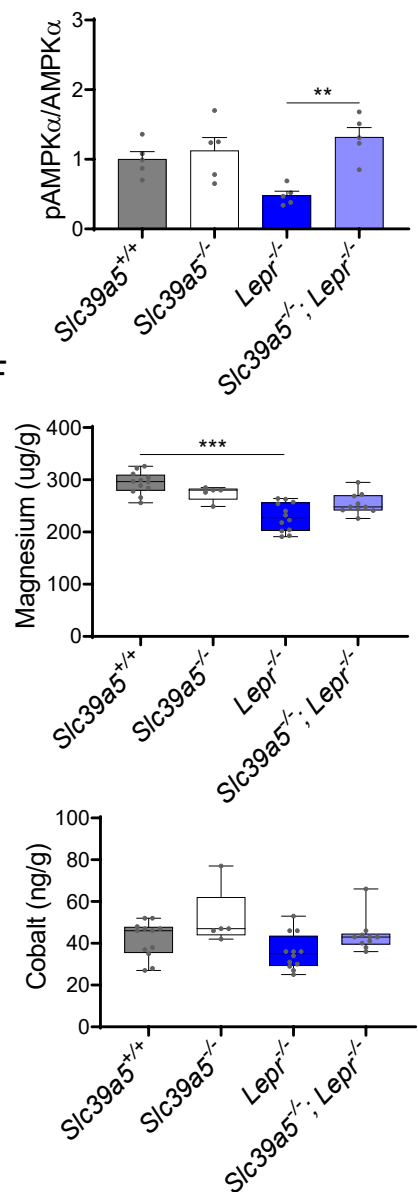
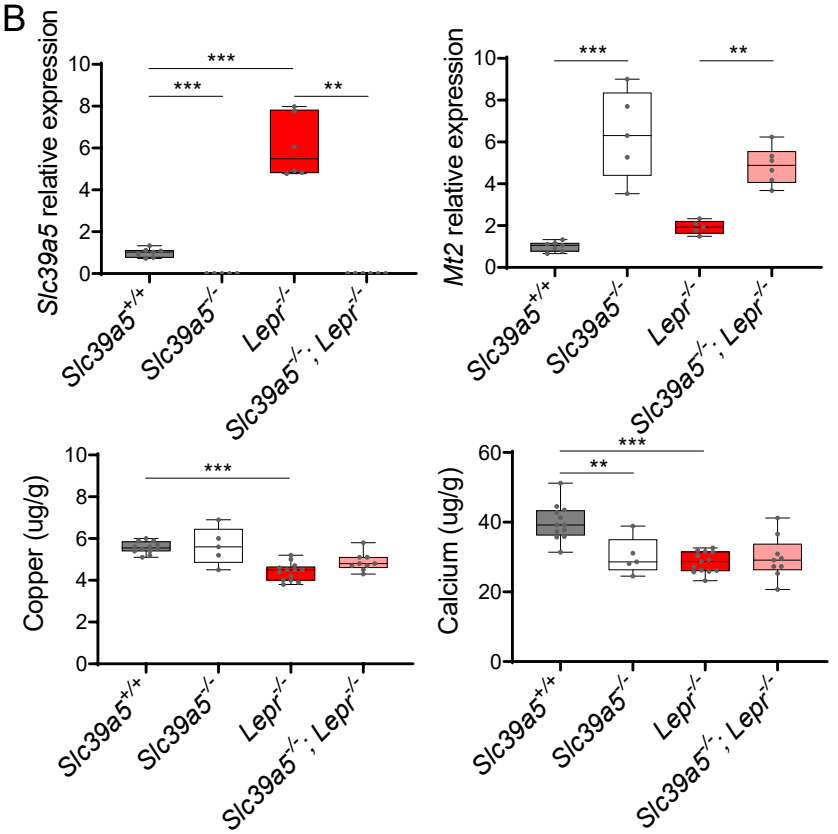

$\mathrm{E}$
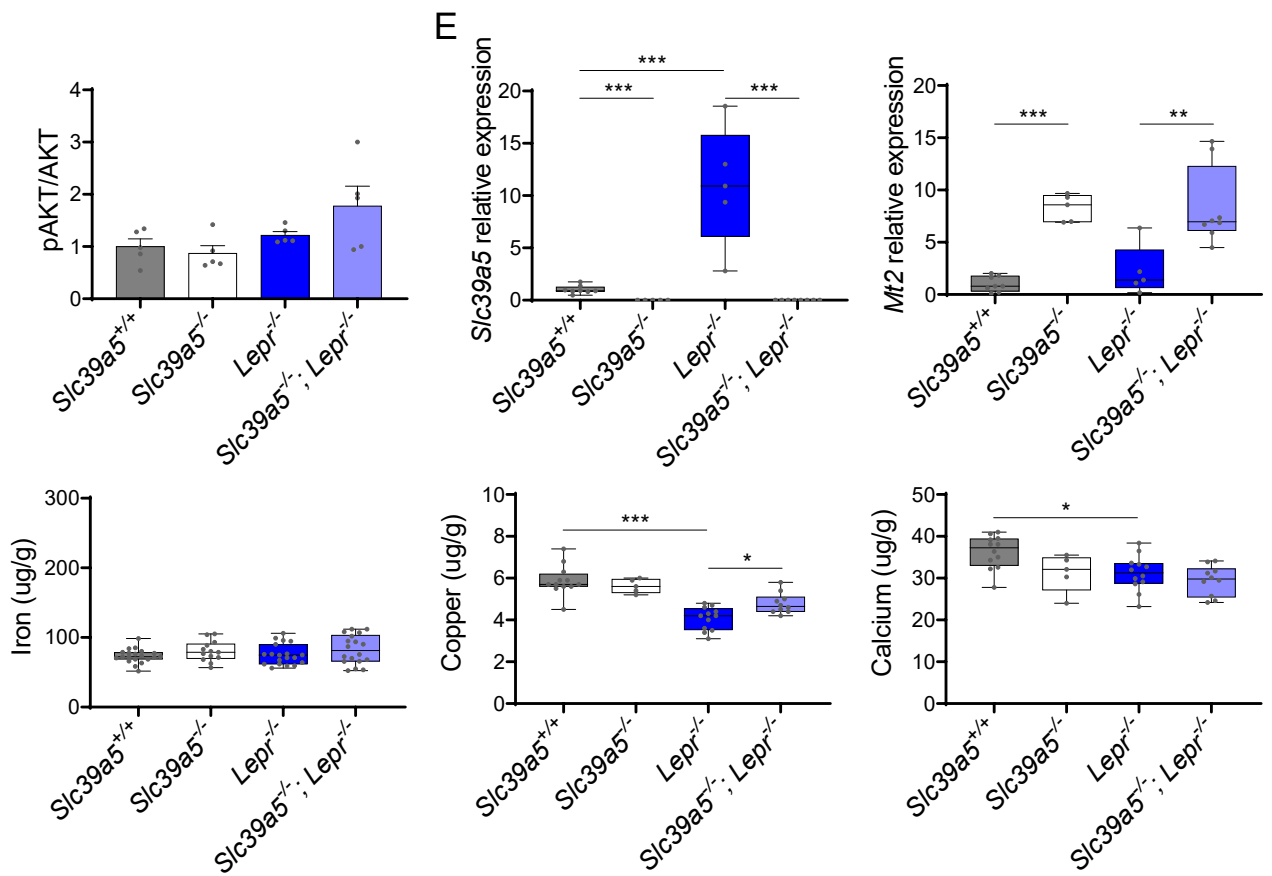

Suppl. Fig. 10. Loss of Slc39a5 does not alter hepatic magnesium, iron, copper, calcium and cobalt levels in Lepr/- mice. Female (A-C) and male (D-F) mice were examined at 34 weeks of age. (A, D) Densitometric analysis of hepatic AMPK and AKT signaling. (B, E) Hepatic expression of Slc39a5 and Mt2. (C, F) Hepatic ion quantification by flame atomic absorption spectrometry. ${ }^{*} \mathrm{P}<0.05$, ${ }^{* *} \mathrm{P}<0.01$, ${ }^{* * *} \mathrm{P}<$ 0.001 , ANOVA with post hoc Tukey's test. 
A $\square$ Slc39a5 $5^{+/+} \square$ Slc39a $5^{-/-}$

q
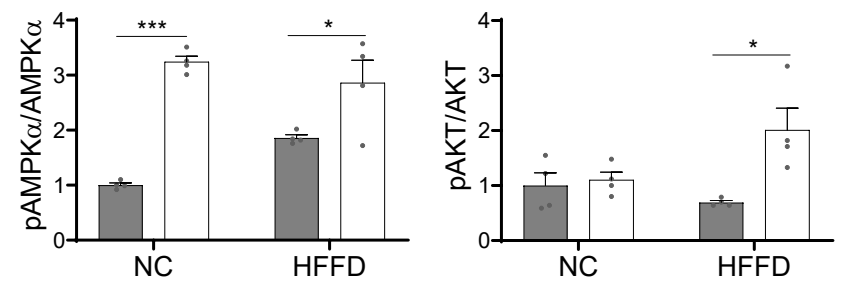

C
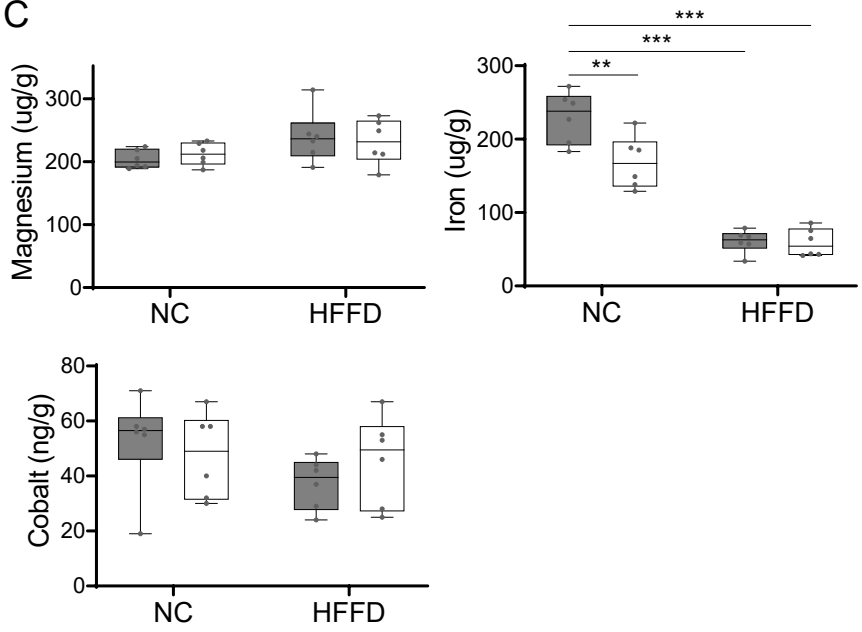

$\mathrm{D}$

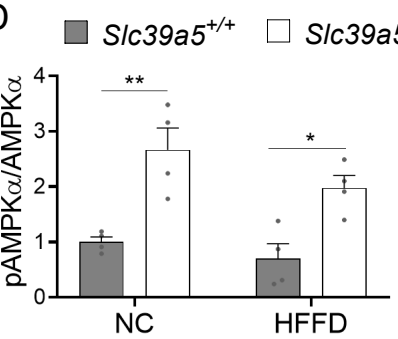

$\mathrm{F}$
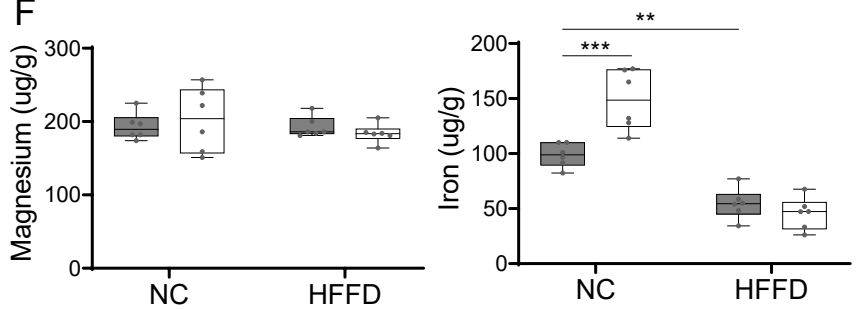
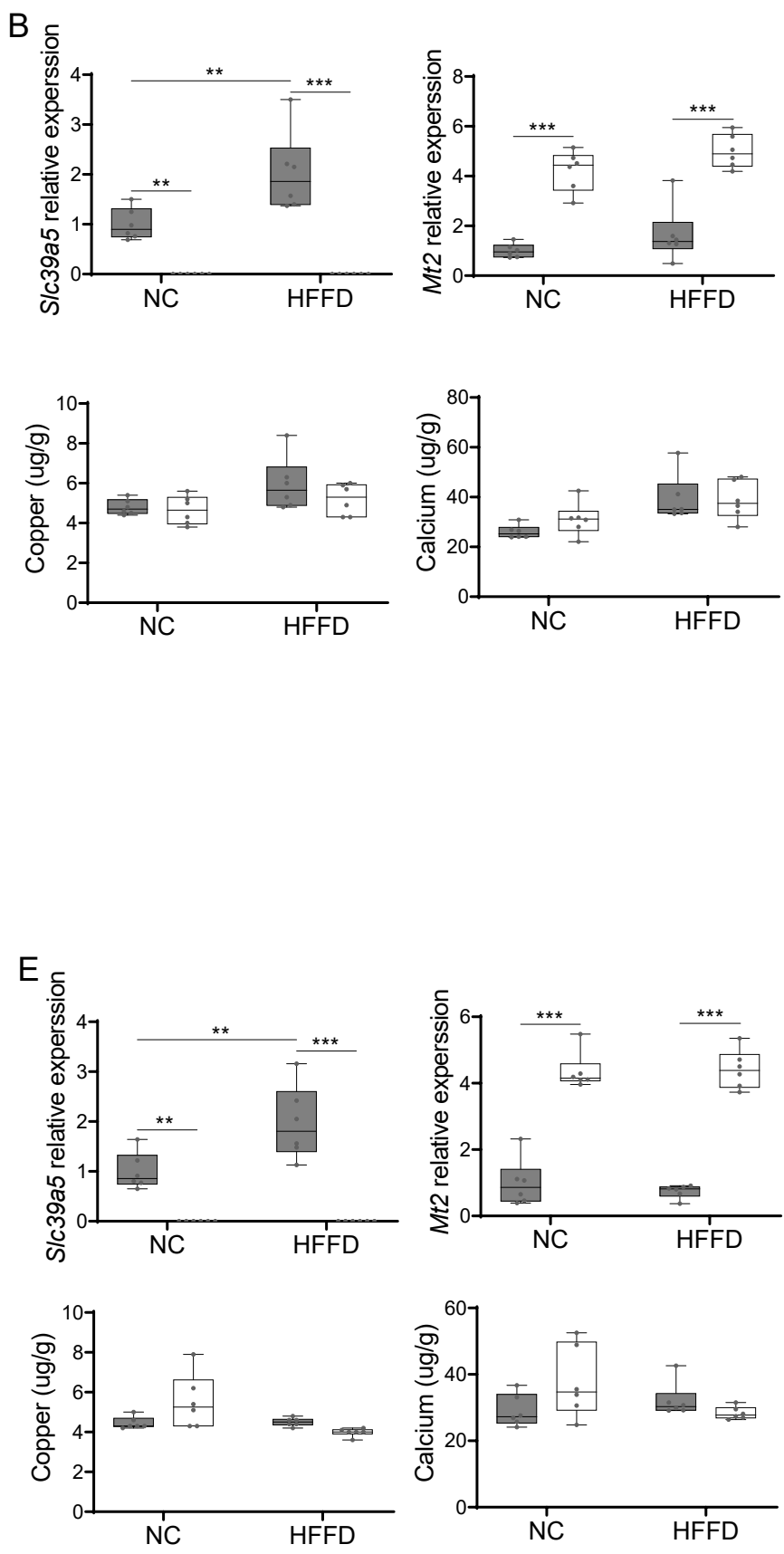

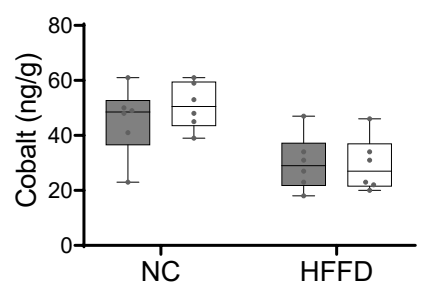

Suppl. Fig. 11. Loss of SIc39a5 does not alter hepatic magnesium, iron, copper, calcium and cobalt levels in mice challenged with high fat high fructose diet (HFFD). Female (A-C) and male (D-F) mice were fed HFFD or NC for 30 weeks. (A, D) Densitometric analysis of hepatic AMPK and AKT. (B, E) Hepatic gene expression of Slc39a5 and Mt2. (C, F) Hepatic ion quantification by flame atomic absorption spectrometry. ${ }^{*} \mathrm{P}<0.05,{ }^{* *} \mathrm{P}<0.01$, ${ }^{* * *} \mathrm{P}<0.001$, two-way ANOVA with post hoc Tukey's test. 
A

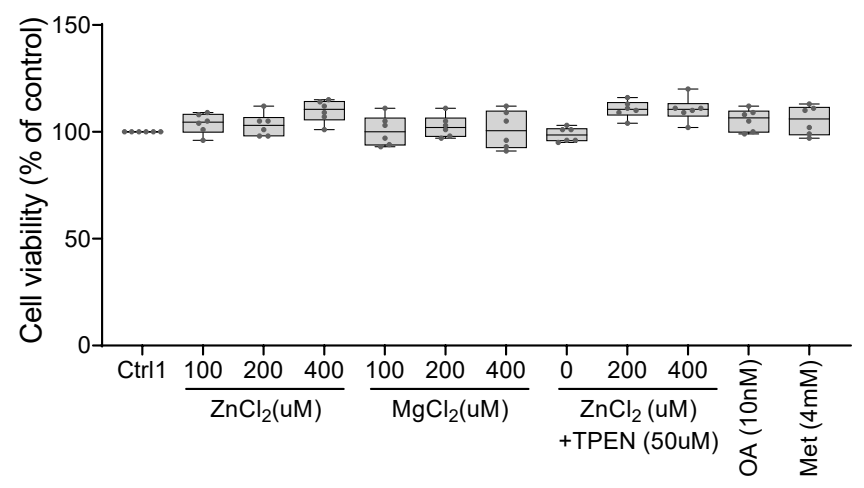

B

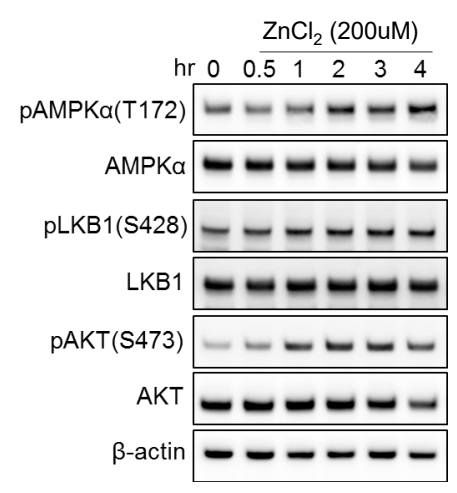

D

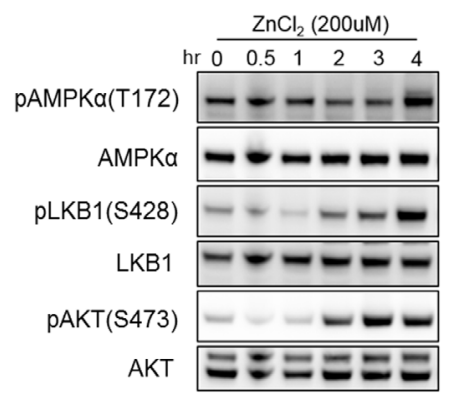

Suppl. Fig. 12. Zinc activates AMPK and AKT signaling in time-dependent and dose-dependent manner. (A) No differences in cell viability observed in human primary hepatocytes (after 4hr treatment) across different experimental groups. (B) Time-resolved (0-4hr) immunoblotting analyses of primary human hepatocytes treated with zinc chloride. (C) Immunoblots of HepG2 treated with zinc chloride $\left(\mathrm{ZnCl}_{2}\right)$ and magnesium chloride $\left(\mathrm{MgCl}_{2}\right)$. (D) Time-resolved $(0-4 \mathrm{hr})$ immunoblotting analyses of HepG2 treated with zinc chloride. Okadaic acid (OA), metformin (Met), N,N,N',N'-Tetrakis(2-pyridylmethyl)ethylenediamine (TPEN). 

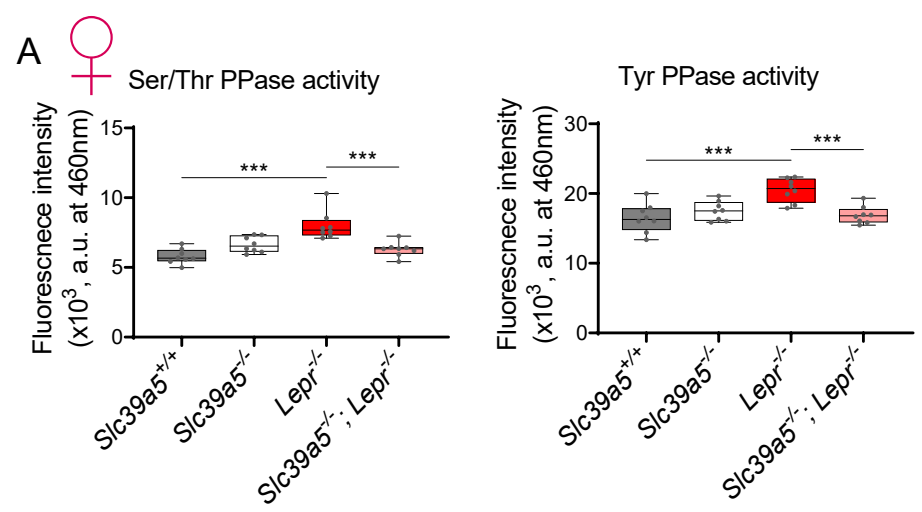

$B \overbrace{\text { Ser/Thr PPase activity }}$
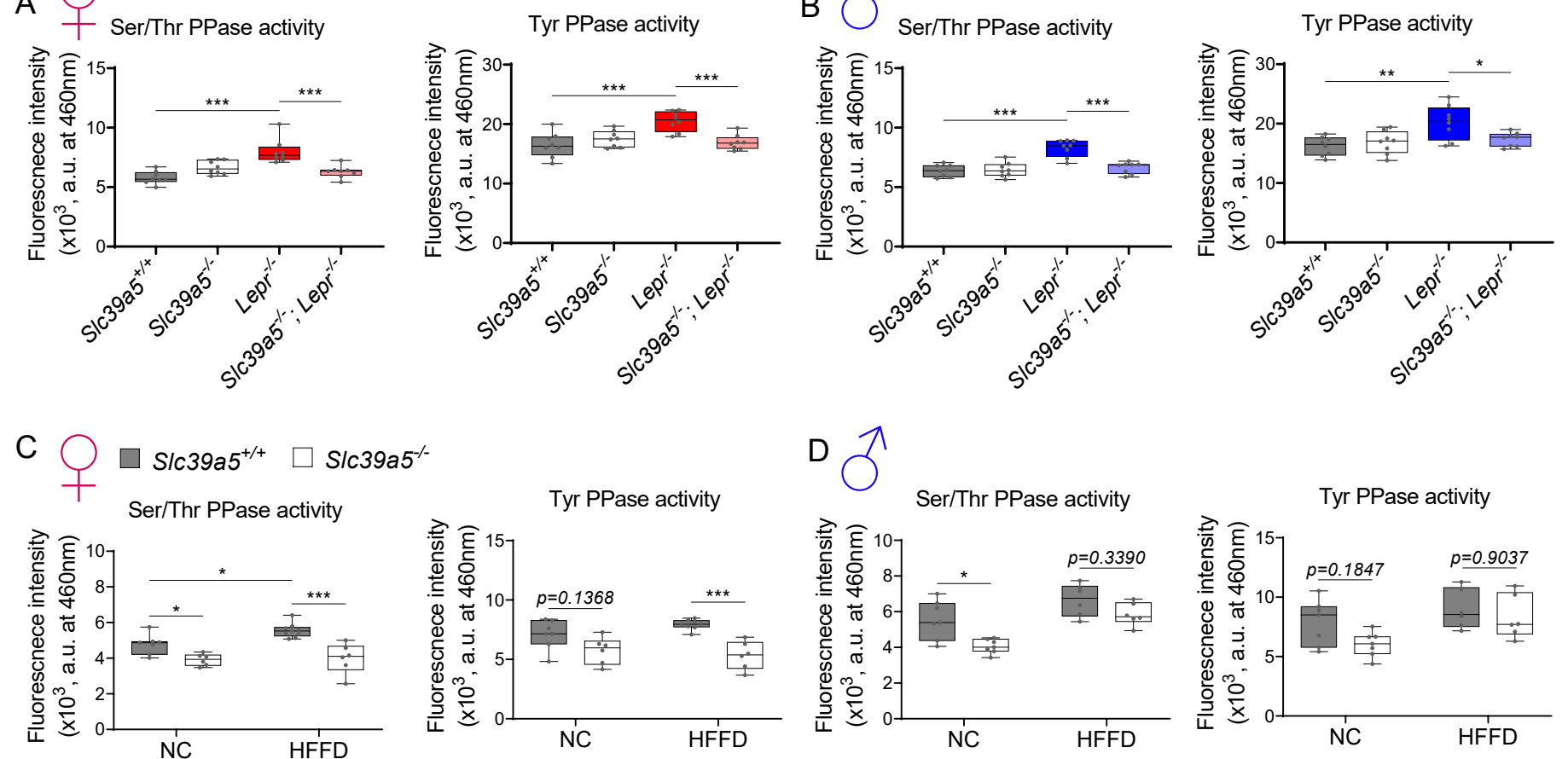

$D$
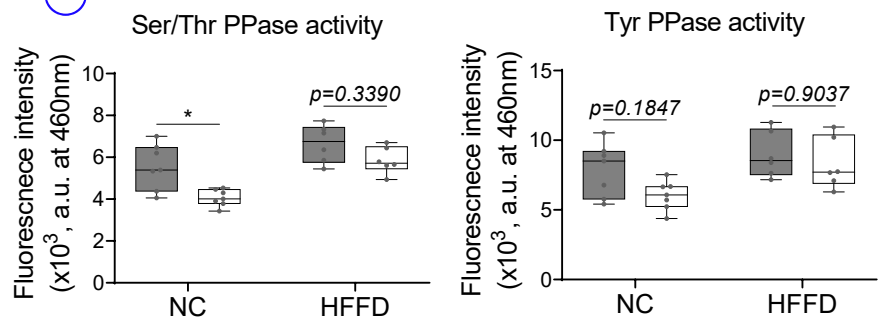

$E$
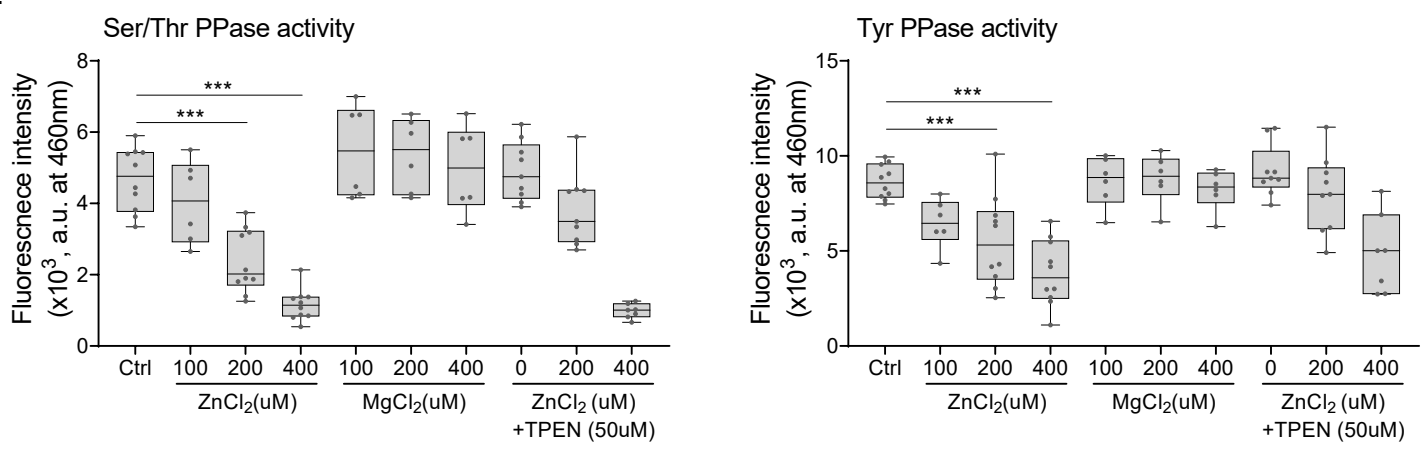

Suppl. Fig. 13. Elevated hepatic zinc results in reduced protein phosphatase activity. Analyses were done on explanted liver samples collected after 16 hour fast at endpoint of congenital obesity (A-B) and diet-induced obesity (C-D) challenges. Female (A, C) and Male (B, D) mice. (A-D) Ser/Thr and Tyr protein phosphatase activity. (E) Ser/Thr and Tyr protein phosphatase activity in primary human hepatocytes treated with zinc chloride $\left(\mathrm{ZnCl}_{2}\right)$, magnesium chloride $\left(\mathrm{MgCl}_{2}\right)$ and $\mathrm{N}, \mathrm{N}, \mathrm{N}^{\prime}, \mathrm{N}^{\prime}$-Tetrakis(2-pyridylmethyl)ethylenediamine (TPEN) for 4 hours. ${ }^{*} \mathrm{P}<0.05,{ }^{* *} \mathrm{P}<0.01,{ }^{* * *} \mathrm{P}<0.001$, ANOVA with post hoc Tukey's test. 


\section{$\mathrm{A} \square \mathrm{S} / \mathrm{c} 39 \mathrm{a} 5^{+/+} \square \mathrm{S} / \mathrm{c} 39 \mathrm{a} 5^{-/} \mathrm{B}$}

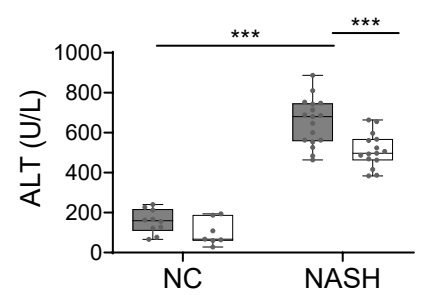

C

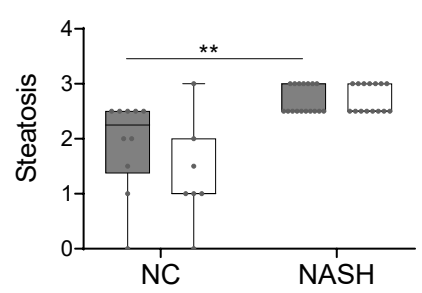

$E$

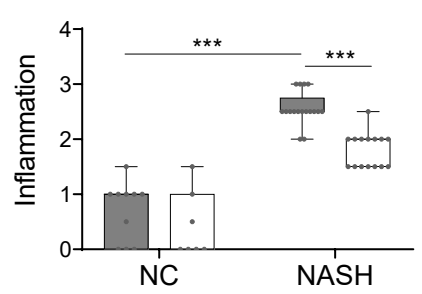

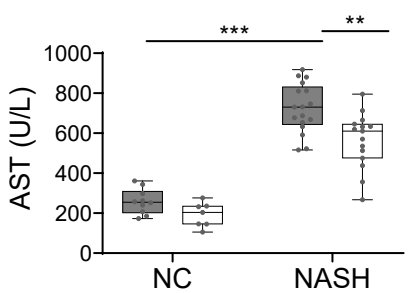

$\mathrm{D}$

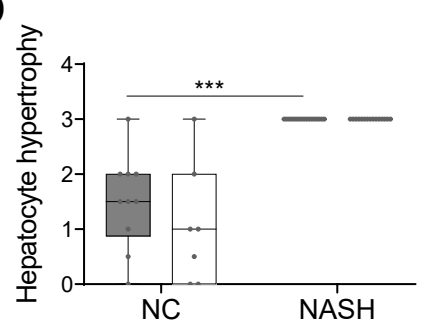

$\mathrm{F}$

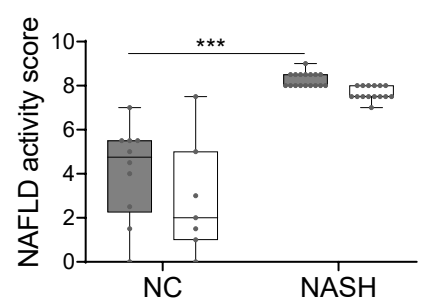

G
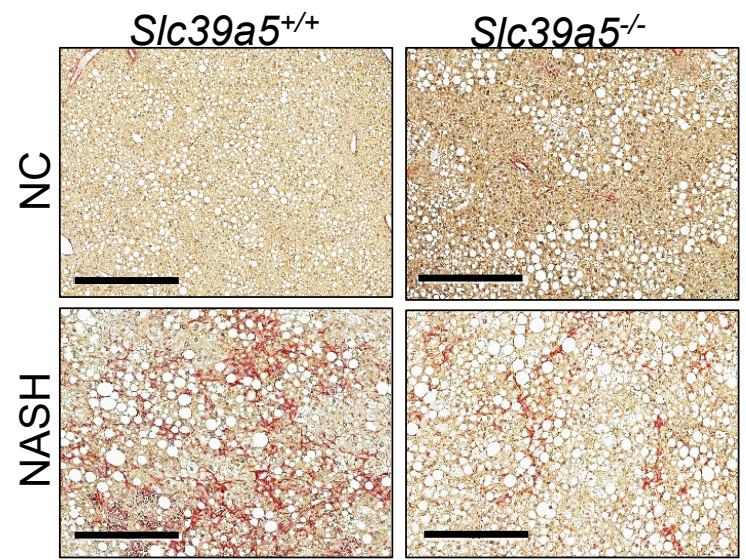

$\mathrm{H}$

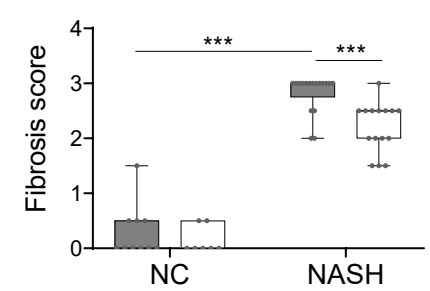

S/c39a5 $\%$

I

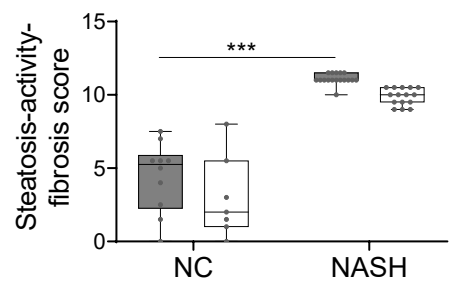

Suppl. Fig. 14. Loss of Slc39a5 reduces hepatic inflammation and fibrosis in male mice challenged with diet-induced NASH. Mice were fed NASH diet or NC for 40 weeks and sacrificed after 16 hour fasting. (A-B) Loss of Slc39a5 reduces serum ALT and AST levels (biomarkers of liver damage). (C-E) Histology scores for steatosis, hepatocyte hypertrophy, inflammation. (F) NAFLD activity score. (G-I) Loss of S/c39a5 improves fibrosis in mice upon NASH dietary challenge. (G) Representative images of explanted livers sample stained with picrosirius red

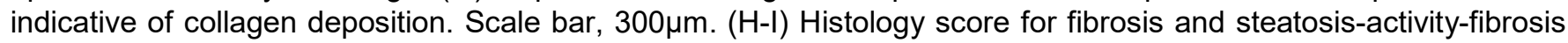
score. $\mathrm{n}=7-10$ (NC) and 15-17 (NASH), ${ }^{*} \mathrm{P}<0.05$, ${ }^{* *} \mathrm{P}<0.01$, ${ }^{* * *} \mathrm{P}<0.001$, two-way ANOVA with post hoc Tukey's test. Numeric data is summarized in suppl. Table 6. 
$\mathrm{A} \square \mathrm{S} / \mathrm{c} 39 \mathrm{a} 5^{+/+} \square \mathrm{S} / \mathrm{c} 39 \mathrm{a} 5^{-/} \mathrm{B}$

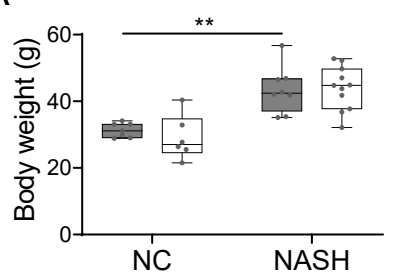

$E$

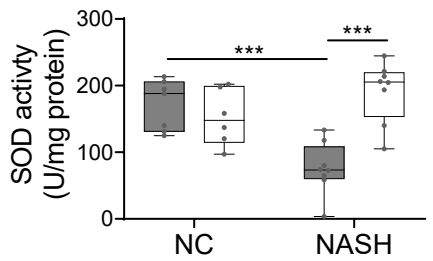

$\mathrm{F}$

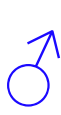

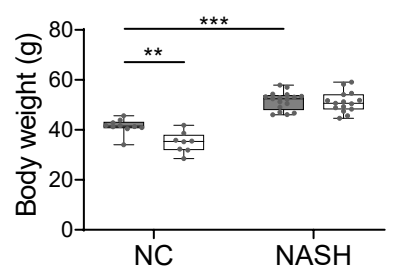

$\mathrm{J}$

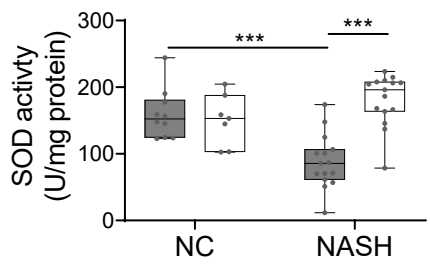

$\mathrm{C}$
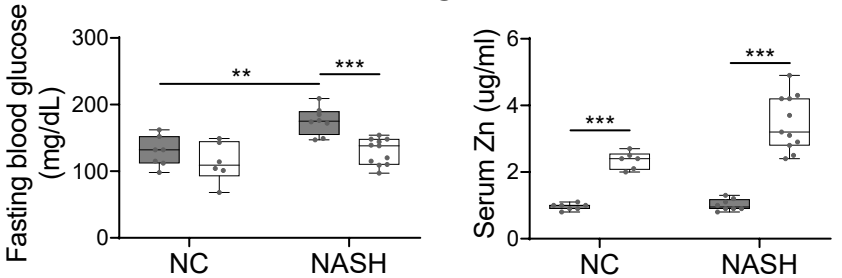

D

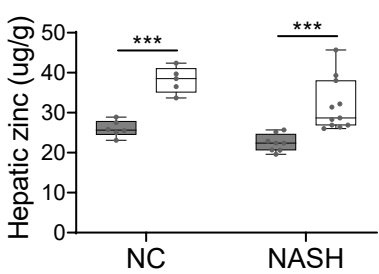

$\mathrm{H}$

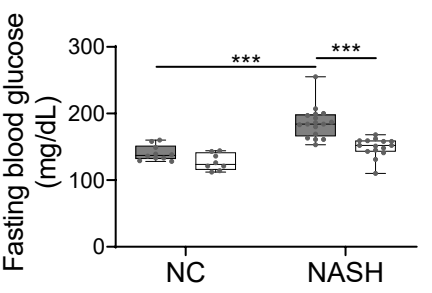

1

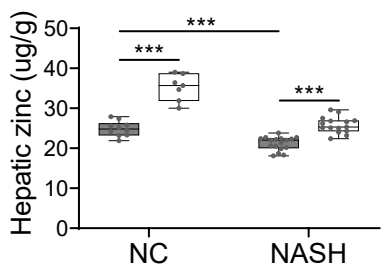

Suppl. Fig. 15. Loss of Slc39a5 improves liver function in mice challenged with diet-induced NASH. Female (A-E) and Male (F-J) mice. (A, F) Body weight. (B, G) Fasting blood glucose. (C, H) Serum zinc. (D, I) Hepatic zinc. (E, J) Total hepatic SOD activity. $n=6-10(\mathrm{NC})$ and 8-17 (NASH), ${ }^{*} P<0.05$, ${ }^{* *} P<0.01,{ }^{* * *} P<0.001$, two-way ANOVA with post hoc Tukey's test. 


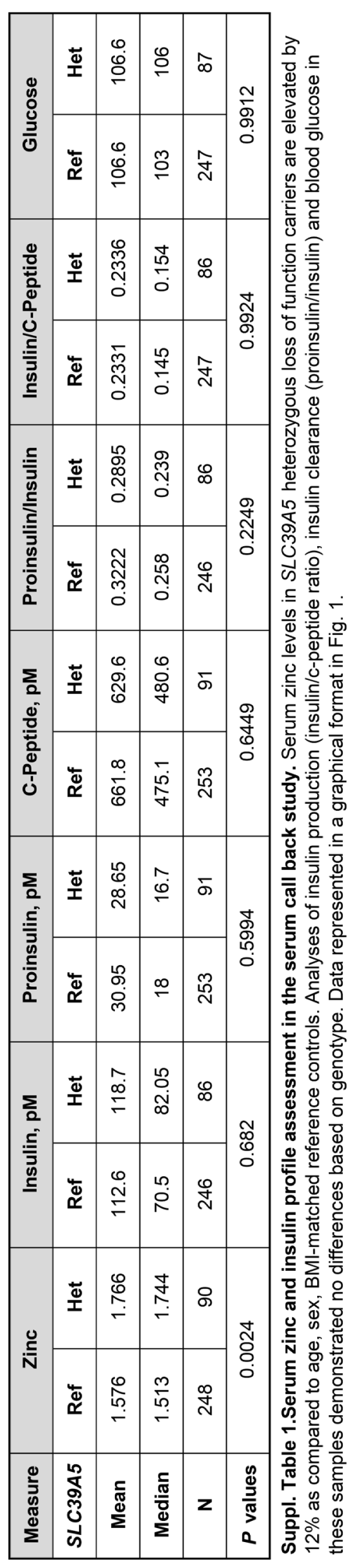




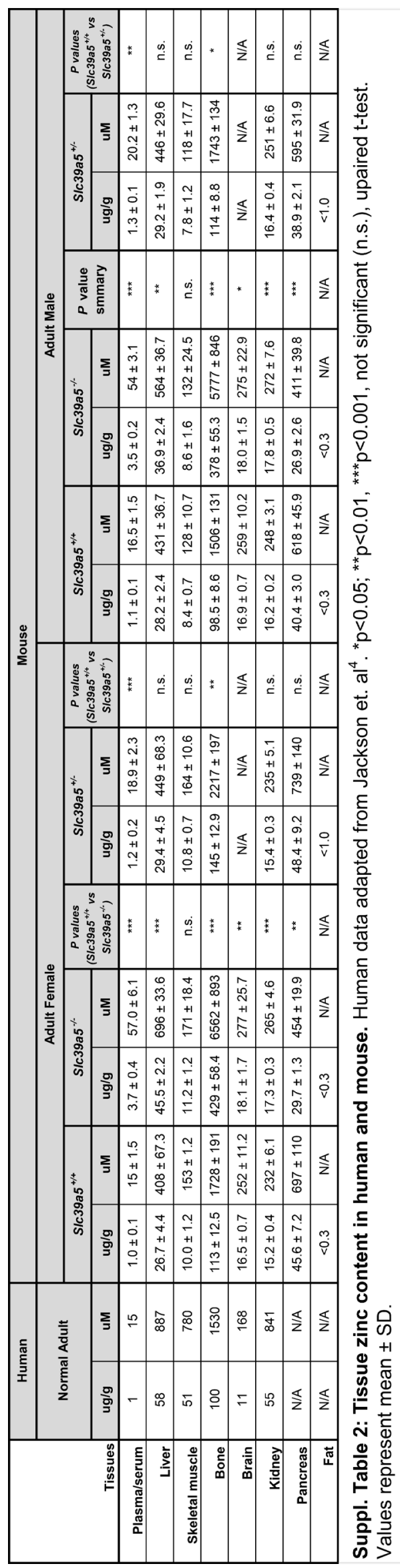




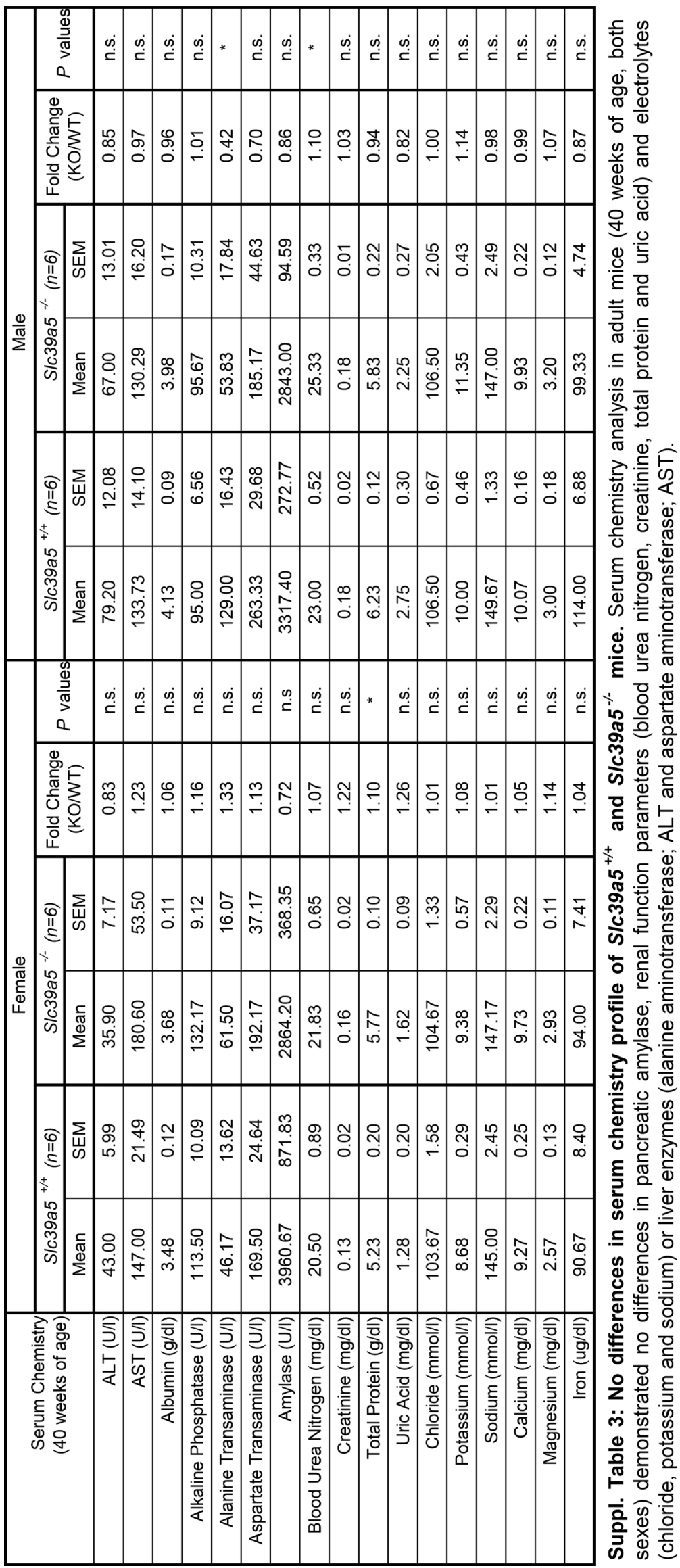




\begin{tabular}{|c|c|c|c|c|c|c|c|c|c|c|c|c|c|c|c|c|c|c|c|c|c|c|}
\hline$\frac{\stackrel{0}{7}}{\frac{N}{2}}$ & 定 & 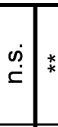 & 类 & $*$ & * & 絭 & * & * & 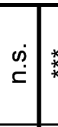 & * & $\stackrel{0}{c}$ & 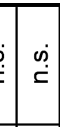 & $\mid \begin{array}{c}\dot{\rho} \\
\dot{\Sigma}\end{array}$ & 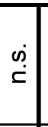 & 粶 & 絭 & * & 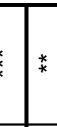 & * & 絭 & 絭 & 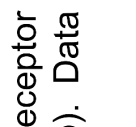 \\
\hline 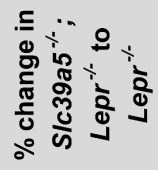 & | & ণे & ஃ̊ & 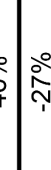 & $\frac{\circ}{\circ 0}$ & ণั & 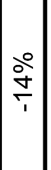 & 总 & ì & ণิ & 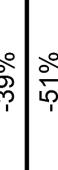 & $\begin{array}{l}0 \\
: \\
\end{array}$ & $\stackrel{\circ}{\circ}$ & 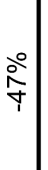 & 今̊ & ১े| & & 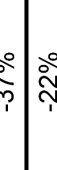 & 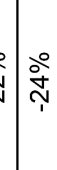 & ¿̊ & $\frac{\circ}{\bar{p}}$ & 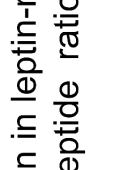 \\
\hline के & $\left|\begin{array}{c}\tilde{N} \\
\stackrel{2}{ } \\
+1 \\
+1 \\
0 \\
0 \\
0 \\
0\end{array}\right|$ & 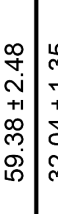 & 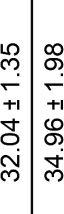 & 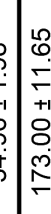 & 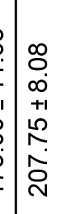 & 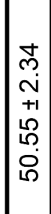 & $\begin{array}{l}\hat{N} \\
\infty \\
+ \\
+1 \\
o \\
o \\
o \\
0 \\
0\end{array}$ & $\left|\begin{array}{c}\hat{\sim} \\
i \\
+1 \\
+1 \\
0 \\
\vdots \\
\dot{y}\end{array}\right|$ & 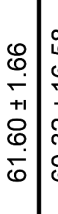 & 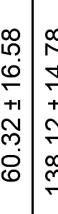 & 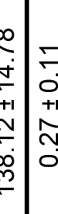 & 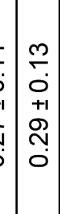 & 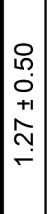 & 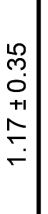 & 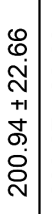 & 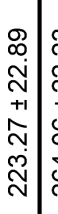 & 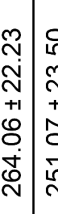 & 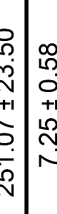 & 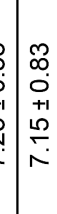 & $\begin{array}{l}\text { N } \\
\text { m } \\
+1 \\
+ \\
\text { N } \\
\text { N }\end{array}$ & $\begin{array}{l}10 \\
0 \\
m \\
+1 \\
\infty \\
o \\
0 \\
10 \\
10\end{array}$ & 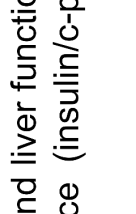 \\
\hline $\begin{array}{l}\frac{0}{2} \\
\frac{2}{3} \\
0\end{array}$ & 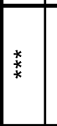 & * & * & * & * & * & $\underset{*}{*} \underset{*}{*}$ & $\underset{*}{*}$ & * & * & ? & 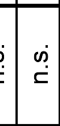 & 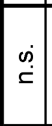 & 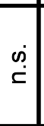 & $\begin{array}{l}\underset{*}{*} \\
* \\
*\end{array}$ & $\underset{*}{*}$ & $\begin{array}{l}* \\
* \\
* \\
*\end{array}$ & $x_{*}^{*}$ & 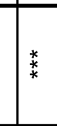 & * & 絭 & 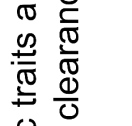 \\
\hline 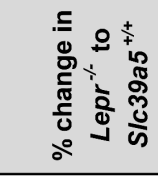 & 今े & 'ે & مे & \&̊ & $\frac{0}{2}$ & $\begin{array}{l}\stackrel{0}{0} \\
\stackrel{0}{m}\end{array}$ & ஓे & $\frac{\circ}{\grave{\rho}}$ & 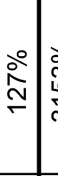 & 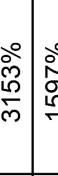 & 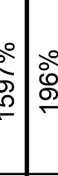 & : & ஓे & ๑े & 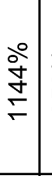 & 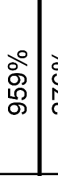 & 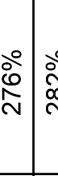 & רָ̀ & 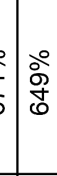 & 餄 & 商 & 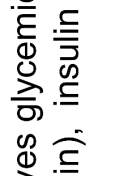 \\
\hline ذ্ & $\left|\begin{array}{c}\stackrel{N}{N} \\
\sim \\
+1 \\
\sim \\
\tilde{N} \\
\stackrel{0}{0}\end{array}\right|$ & 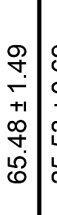 & 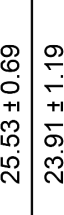 & 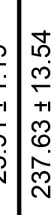 & 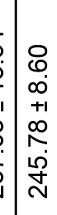 & 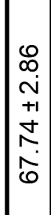 & $\begin{array}{l}1 \\
0 \\
i \\
+1 \\
0 \\
0 \\
m \\
0 \\
0\end{array}$ & \begin{tabular}{|c|c|}
$\Gamma$ \\
$T$ \\
+1 \\
+1 \\
0 \\
0 \\
0 \\
0
\end{tabular} & 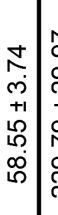 & 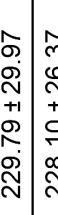 & 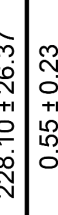 & 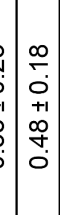 & $\mid \begin{array}{c}m \\
\check{0} \\
+1 \\
+1 \\
0 \\
\stackrel{-}{-}\end{array}$ & $\begin{array}{l}\infty \\
+ \\
0 \\
+1 \\
+1 \\
\\
\end{array}$ & 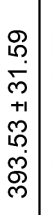 & 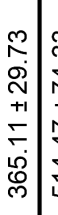 & 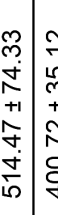 & 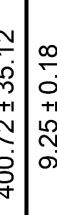 & 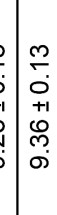 & 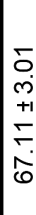 & 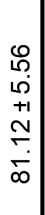 & 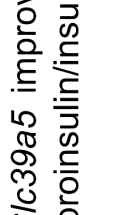 \\
\hline$\frac{9}{\frac{0}{50}}$ & $\stackrel{\dot{\varphi}}{\dot{c}}$ & $\stackrel{\dot{\rho}}{\check{c}}$ & * & . & 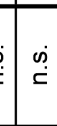 & $\stackrel{\dot{\oplus}}{\dot{x}}$ & $\mid \stackrel{\dot{\rho}}{\dot{\Sigma}}$ & $\stackrel{\dot{\varphi}}{\dot{c}}$ & $\stackrel{\dot{\varphi}}{\dot{c}}$ & $\stackrel{\dot{p}}{\dot{c}}$ & | & $\stackrel{\dot{\rho}}{\check{c}}$ & $\stackrel{\dot{\varphi}}{\dot{\varphi}}$ & 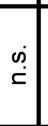 & $\stackrel{\dot{\varphi}}{\dot{\varphi}}$ & $\stackrel{\dot{\oplus}}{\dot{\check{c}}}$ & $\stackrel{\dot{m}}{\dot{x}}$ & | & 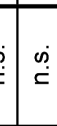 & & $\stackrel{\dot{m}}{\dot{\epsilon}}$ & 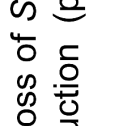 \\
\hline 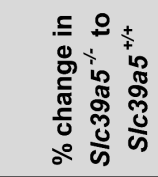 & ڤे & |ڤ & $\begin{array}{l}\circ \\
\grave{\sigma} \\
\grave{\sigma}\end{array}$ & $\stackrel{\circ}{\circ}$ & ळे & ج̊ & $\frac{2}{1}$ & 仓े & ণे & ஓें & 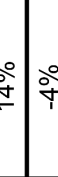 & $\begin{array}{l}8 \\
f \\
+\end{array}$ & 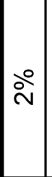 & 守 & 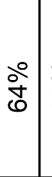 & ১े & ○े & ְे & ठ̀ & 웡 & 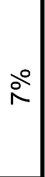 & 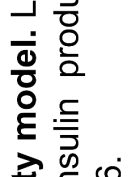 \\
\hline $\begin{array}{l}\text { कू } \\
\text { कृ } \\
\text { फ }\end{array}$ & 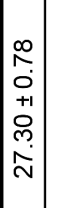 & 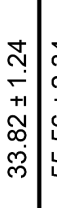 & 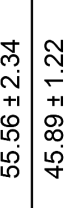 & 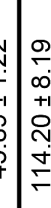 & $\begin{array}{l}0 \\
0 \\
T \\
+1 \\
0 \\
0 \\
0 \\
\mathbb{1} \\
0 \\
0\end{array}$ & 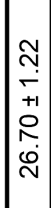 & $\mid \begin{array}{l}0 \\
0 \\
0 \\
+1 \\
0 \\
0 \\
\infty \\
\infty \\
0\end{array}$ & 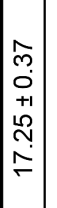 & 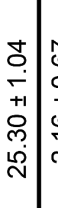 & 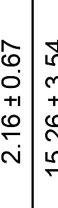 & 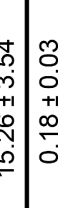 & 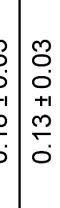 & $\mid \begin{array}{l}2 \\
0 \\
0 \\
+1 \\
+1 \\
L \\
L \\
0 \\
0\end{array}$ & $\begin{array}{l}\stackrel{0}{m} \\
0 \\
+1 \\
+1 \\
\stackrel{1}{2} \\
- \\
-\end{array}$ & 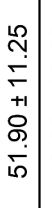 & $\begin{array}{c}8 \\
\dot{m} \\
\dot{m} \\
+1 \\
+1 \\
0 \\
0 \\
\dot{\sigma} \\
\dot{\sigma}\end{array}$ & 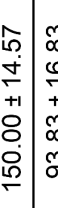 & 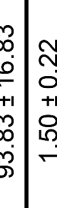 & 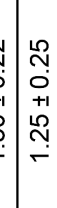 & 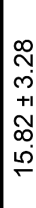 & 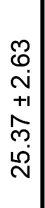 & 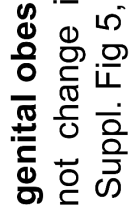 \\
\hline 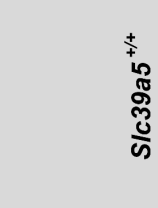 & 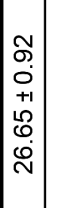 & 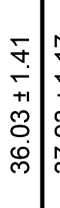 & 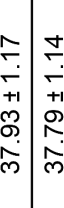 & 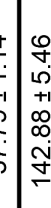 & 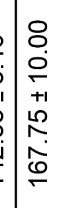 & $\begin{array}{l}0 \\
\infty \\
0 \\
0 \\
+1 \\
\tilde{O} \\
0 \\
0 \\
\infty \\
\tilde{N}\end{array}$ & 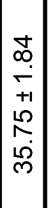 & \begin{tabular}{|c|}
$\bar{T}$ \\
0 \\
+1 \\
+1 \\
$\infty$ \\
$\infty$ \\
$\infty$ \\
$\infty$ \\
\end{tabular} & 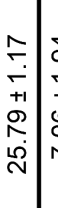 & 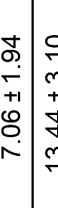 & 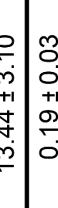 & 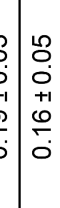 & $\left|\begin{array}{c}\mathfrak{N} \\
\check{0} \\
+1 \\
+1 \\
\tilde{S} \\
0 \\
0\end{array}\right|$ & 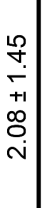 & 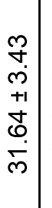 & 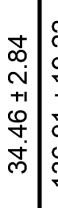 & 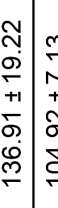 & 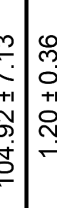 & 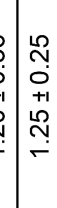 & مִ & 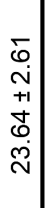 & 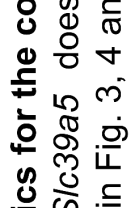 \\
\hline$\stackrel{\times}{\circledR}$ & ४ & $\Sigma$ & $4 \mid \Sigma$ & ५ & $\Sigma$ & ᄂ & $\Sigma$ & 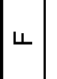 & $\Sigma$ & $\leftarrow$ & ४ & 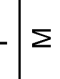 & ४ & $\Sigma$ & ч & $\Sigma$ & $\leftarrow$ & $\Sigma$ & $\mid \Sigma$ & 4 & $\Sigma$ & 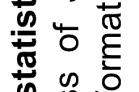 \\
\hline & 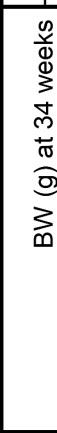 & कृ & 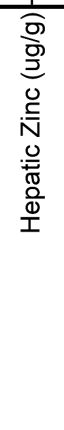 & & 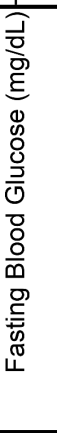 & , & 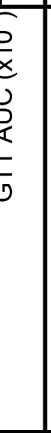 & $\begin{array}{l}\tilde{n} \\
\frac{0}{x} \\
0 \\
0 \\
\frac{1}{2} \\
E\end{array}$ & & $\begin{array}{l}\frac{\alpha}{1} \\
\sum \\
\frac{1}{2} \\
\text { 온 }\end{array}$ & & 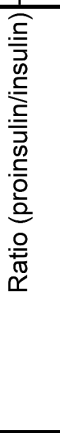 & 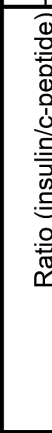 & & $\stackrel{5}{\frac{5}{\varangle}}$ & & 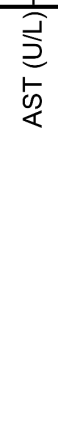 & & 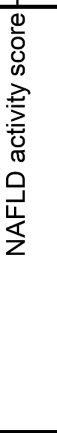 & & בְ & 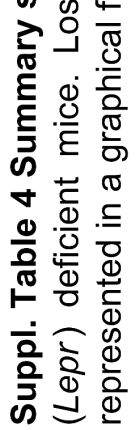 \\
\hline
\end{tabular}




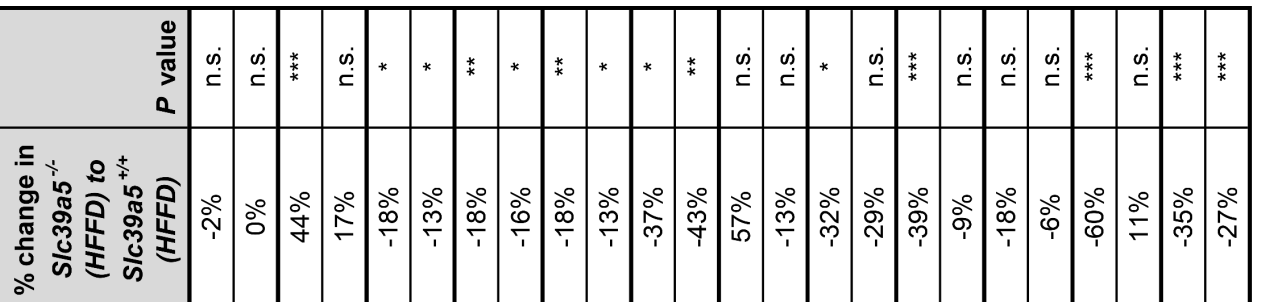

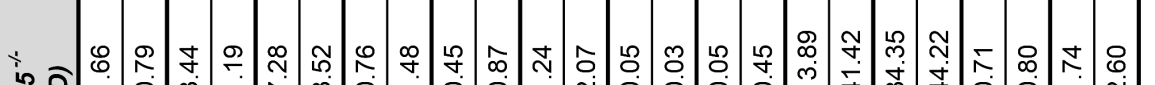

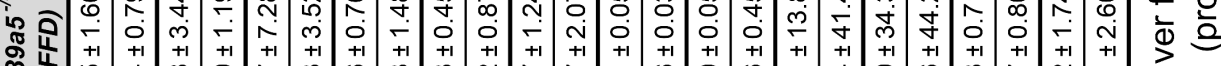

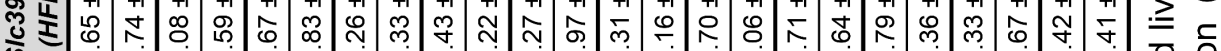

ज 守

ㄴ

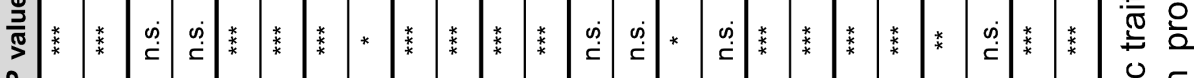

Q

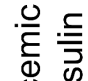

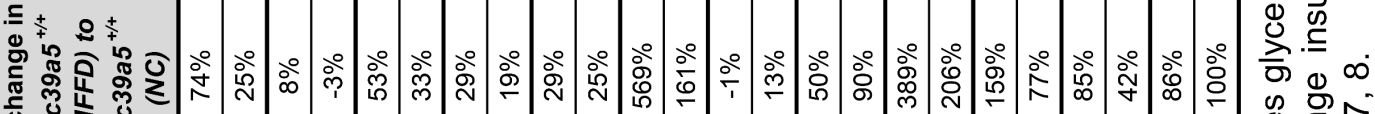

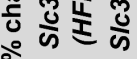

t.

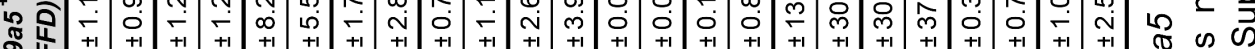

空

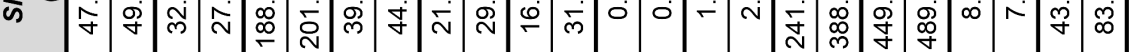

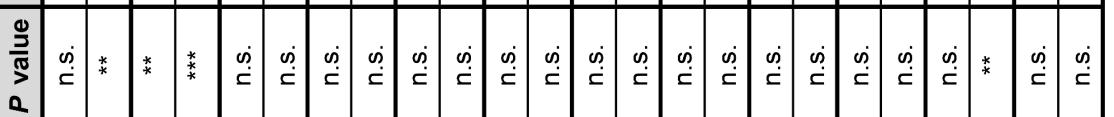

心

ப

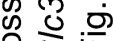

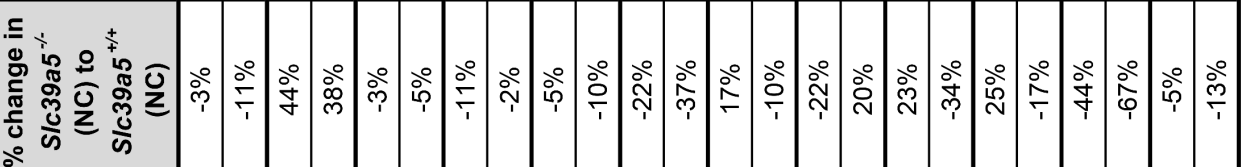

-

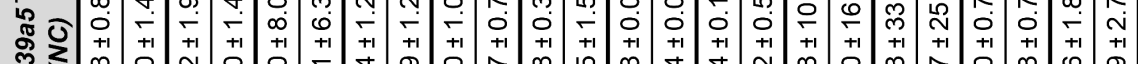

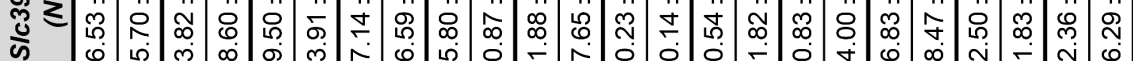

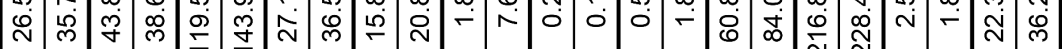

的

훙

\%

드 흠

产㐫

\$ 응 응

은 휴

$\Phi<\sigma$

음

등 음

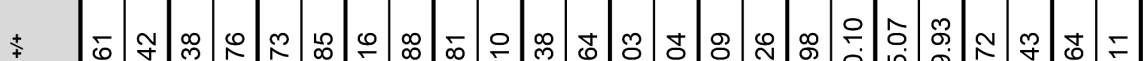

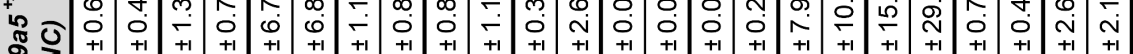

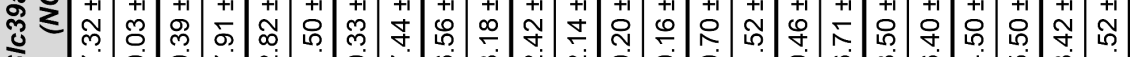

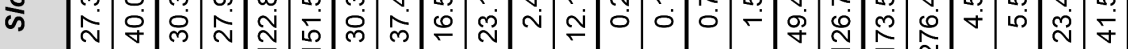

\begin{tabular}{|c|c|c|c|c|c|c|c|c|c|c|c|c|c|c|c|}
\hline$\llcorner\Sigma$ & ४ $\Sigma$ & ४ $\mid \Sigma$ & 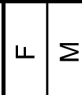 & ४ & $\Sigma L$ & $4 \mid \Sigma$ & 4 & $\mid$\begin{tabular}{l|l} 
& $\Sigma$
\end{tabular} & 4 & 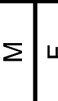 & $4 \mid \Sigma$ & 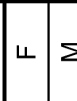 & ४ & $\Sigma$ & 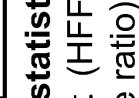 \\
\hline 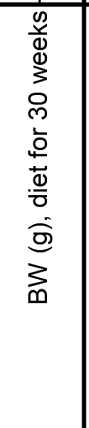 & 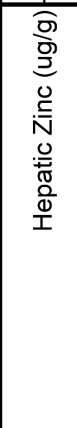 & 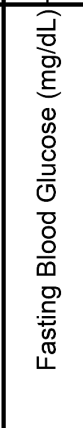 & 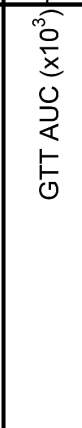 & 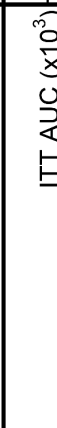 & & $\begin{array}{l}\frac{\alpha}{1} \\
\sum^{\frac{1}{2}} \\
\text { 오 }\end{array}$ & 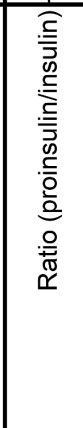 & 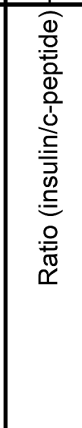 & $\underset{\frac{1}{2}}{\stackrel{5}{ᄅ}}$ & & 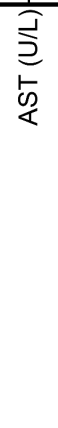 & 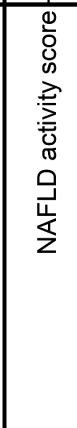 & 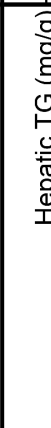 & & 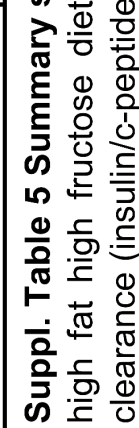 \\
\hline
\end{tabular}




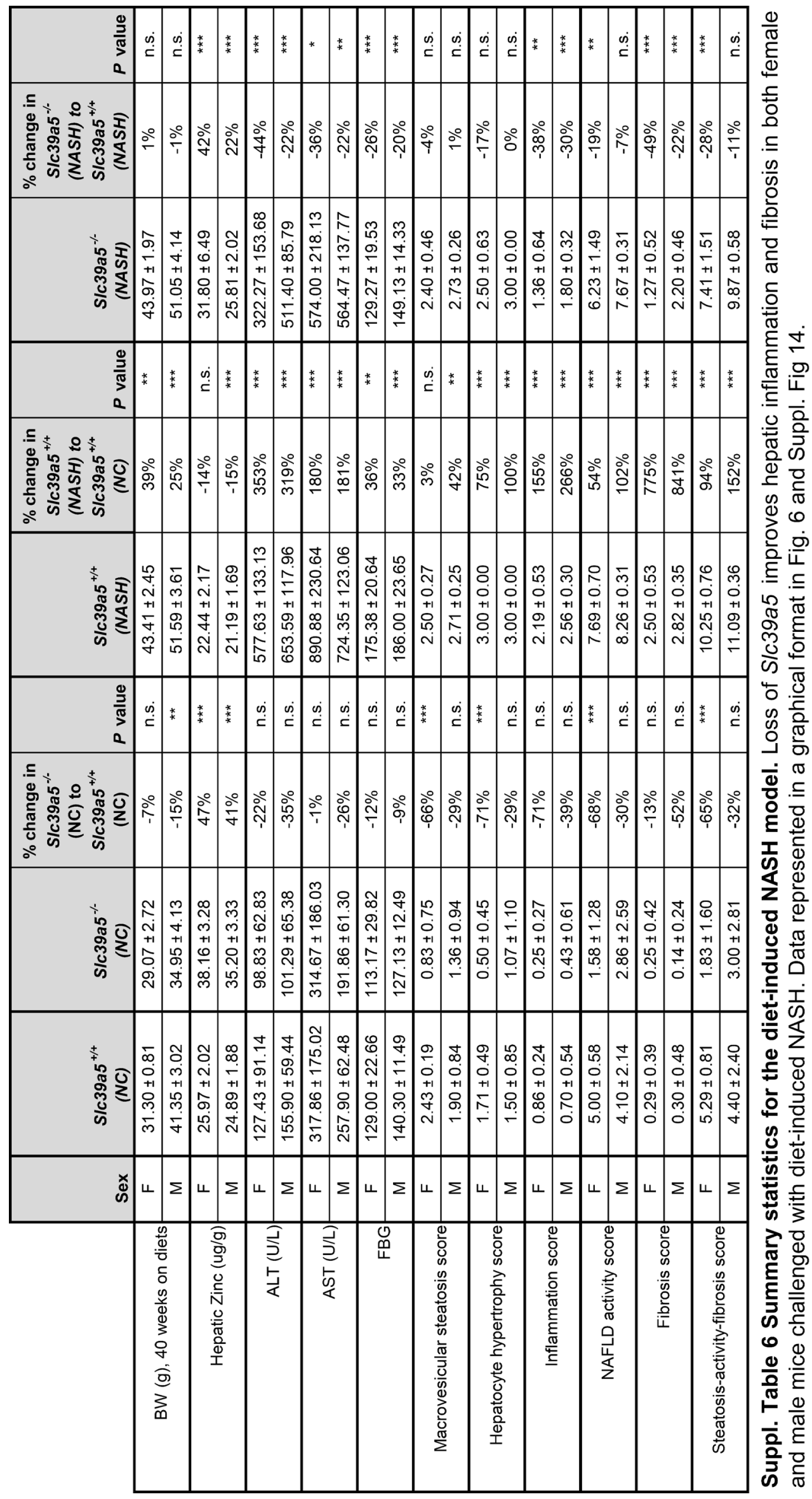

